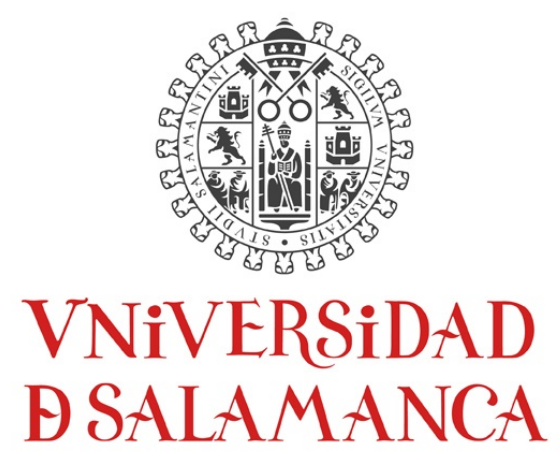

Tesis doctoral

\title{
Color-Kinematics Duality and the Regge Limit
}

Eduardo Serna Campillo 



\title{
Color-Kinematics Duality and the Regge Limit
}

\author{
MEMORIA PRESENTADA POR D. EDUARDO SERNA CAMPILLO \\ PARA OPTAR AL TÍTULO DE DOCTOR EN FÍSICA
}

Director: Miguel Ángel Vázquez Mozo Co-Director: Agustín Sabio Vera 

D. MigUEL ÁNGEL VÁZQUEZ MOZO, Profesor Titular de Universidad del Departamento de Física Fundamental de la Universidad de Salamanca y D. AGUSTÍN SABIO VERA, Profesor Contratado Doctor del Departamento de Física Teórica de la Universidad Autónoma de Madrid

Autorizan la presentación de la memoria de tesis titulada "Color-Kinematics Duality and the Regge Limit", realizada bajo su dirección, por D. EDUARDO SERNA CAMPILLO.

Salamanca, 30 de Septiembre de 2014 



\section{Abstract}

The starting point of this thesis is the calculation of the five-point amplitude for the scattering of two distinct scalars with the emission of one graviton in the final state for Einstein-Hilbert gravity. We find that the result, expressed in Sudakov variables, corresponds to the sum of two gauge invariant contributions written in terms of a new two scalar - two graviton effective vertex. A similar calculation is carried out in Quantum Chromodynamics (QCD) for the scattering of two distinct quarks with one extra gluon in the final state. The effective vertices which appear in both cases are then evaluated in the multi-Regge limit (where the emitted on-shell graviton is well separated in rapidity from the scalar particles) reproducing the well-known result obtained by Lipatov where the Einstein-Hilbert graviton emission vertex from two off-shell gravitons can be related to the product of two QCD gluon emission vertices. After this we investigate tree-level five-point amplitudes in scalar-QCD (sQCD) expressed in terms of Sudakov variables and find the equivalent "gravitational" counterparts using the so-called color-kinematics duality proposed by Bern, Carrasco, and Johannson. Taking the multi-Regge limit in the gravitational amplitudes, we show that those pieces in the coupling of two Reggeized gravitons to one on-shell graviton directly stemming from the double copy of the vertex for two Reggeized gluons to one on-shell gluon are universal and properly reproduced by the duality.

In the second part of the thesis we study the applicability of the colorkinematics duality to the scattering of two now distinguishable scalar matter particles with gluon emission in QCD, or graviton emission in Einstein gravity. Our previous analysis suggested that a direct use of the Bern-Carrasco-Johansson double-copy prescription to matter amplitudes does not reproduce the gravitational amplitude in multi-Regge kinematics. However, minimal extensions of the 
calculation eliminate this obstacle. Here we present two approaches: introduction of a scalar contact interaction, or relaxing distinguishibility of the scalars. In both cases new diagrams allow for a full reconstruction of the correct multi-Regge limit on the gravitational side. Both modifications correspond to pure theories obtained by dimensional reduction from higher-dimensional gauge theories. 


\section{Acknowledgements}

I would like to thank:

My advisors Agustín and Miguel Ángel, for their patience, generosity, common sense, and useful life lessons.

Professor Zvi Bern and his group for extending an invitation to spend some time with his group, the fruitful discussions and teaching me the ropes of a fascinating topic.

German Rodrigo and LHCPhenonet for its financial support, which has enriched my postgraduate experience.

Wolfram Research, and in particular Oleksandr Pavlyk for the wonderful time I spent there, which opened my eyes to many interesting areas of Mathematics.

Henrik Johansson for his invaluable collaboration.

Instituto de Física Teórica UAM/CSIC for giving me a place to work in Madrid.

This work has been supported by a Spanish Government FPI Predoctoral Fellowship and grant FIS2009-07238.

Quiero dar las gracias a:

Mi familia por su amor y apoyo, todos lo bueno en mí lo saco de ellos. Mi abuelo es el ser humano más desinteresado que conozco. Mi madre por su infinito sacrificio e inteligencia. Mi padre por enseñarme su amor por la naturaleza, los animales y el mar. Mis hermanos por ser mis amigos.

Mis amigos por ser increíble cada uno a su manera, no valoro la compañía en si misma, pero el valor que aportan a mi vida y a la de quienes les rodean es incalculable.

Andrea por todo, no tengo palabras.

Para todos los que han sido buenos conmigo. Ser una buena persona es lo 
más (único) importante en la vida. 


\section{Contents}

1 Gauge Theories, Gravity, the Regge Limit, and their Relations 1

1.1 Weak field expansion of Einstein-Hilbert gravity. . . . . . . . . . . 2

1.2 Perturbation theory at high energies and the need for resummation 5

1.3 Color Kinematics Duality and BCJ . . . . . . . . . . . . . . . 9

1.3 .1 Numerators that obey BCJ . . . . . . . . . . . . . . . 13

1.3 .2 BCJ at loop level . . . . . . . . . . . . . . . 16

2 Perturbative Einstein-Hilbert Coupled to Scalars and the Regge

$\begin{array}{lr}\text { Limit } & 19\end{array}$

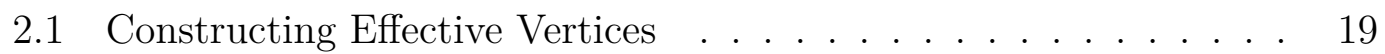

$2.2 \quad$ Feynman rules for perturbative quantum gravity . . . . . . . . . . 20

2.3 Quark-quark scattering with gluon emission . . . . . . . . . . . 24

2.4 Multi-Regge kinematics . . . . . . . . . . . . . . . . . . . . . . . . 28

2.5 Scalar-scalar scattering with graviton emission . . . . . . . . . . . 32

$2.6 \quad$ Gravity as a double copy of QCD in multi-Regge kinematics . . . 37

3 Color-Kinematics Duality and Scalar QCD 43

3.1 Extending the BCJ prescription . . . . . . . . . . . . . . . . 43

$3.2 \quad$ sQCD and color-kinematics duality . . . . . . . . . . . . . 46 
$4 \quad$ BCJ and Dimensional Reduction 59

$4.1 \quad$ Solving the puzzle . . . . . . . . . . . . . . . . . . . . . . . . 59

4.2 Color-kinematics duality with scalar matter . . . . . . . . . . 60

4.2 .1 Distinguishable scalars. . . . . . . . . . . . . . . 65

4.2 .2 Indistinguishable scalars. . . . . . . . . . . . . . . . . . 68

4.3 The role of dimensional reduction . . . . . . . . . . . . . . . 70

$\begin{array}{lll}5 & \text { Conclusions and Outlook } & 73\end{array}$

\begin{tabular}{|ll}
\hline A Explicit Form of The Gravitational Amplitude & 77
\end{tabular}

A.1 The full five-point amplitude . . . . . . . . . . . . . . . . 77

A.2 The two topologies contributing to the full amplitude . . . . . . . 78 


\section{Chapter 1}

\section{Gauge Theories, Gravity, the Regge Limit, and their Relations}

In recent years there has been impressive progress in the understanding of the structure of scattering amplitudes in gauge theories mainly motivated by collider phenomenology but also by the anti de Sitter / conformal field theory (AdS/CFT) correspondence [1, 2, 3]. The latter has boosted the activity towards calculations in the $\mathcal{N}=4$ supersymmetric Yang-Mills (SYM) theory, which enjoys four dimensional conformal invariance and allows for calculations up to a large number of quantum loops by reducing the problem to a small set of master topologies [4]. These results can then be directly used to obtain amplitudes in $\mathcal{N}=8$ supergravity, offering the possibility to investigate the renormalizability of the theory at high orders in the gravitational coupling [5, 6].

However, there are some cases, like Einstein-Hilbert gravity [7, 8, 9], where supersymmetry and string theory based calculational techniques are of little help [10, 11, 12]. In this case one is forced to approach the calculations with traditional Feynman rules [13, 14, 15]. At each order of perturbation theory the task at hand grows tremendously due to the new multi-graviton vertices appear- 
ing and the problem becomes a combinatorial nightmare. To make progress in this direction it is needed to find simplifying techniques besides using computer algebra.

\subsection{Weak field expansion of Einstein-Hilbert grav- ity.}

Our target is to perform quantum calculations in gravity using perturbation the-

ory in the Newton constant $\kappa=\sqrt{\frac{8 \pi G}{c^{2}}}$. Since in gravity the field corresponds to the metric, it is convenient to consider contributions around a flat background (given by the Minkowski metric $\eta_{\mu \nu}$ ). This will allow us to define a graviton as the remaining perturbation to the metric $\eta_{\mu \nu}$.

In more detail, we introduce the expansion $g_{\mu \nu}=\eta_{\mu \nu}+\kappa h_{\mu \nu}$ in the EinsteinHilbert action

$$
S=\int d^{4} x \frac{\sqrt{-g}}{2}\left(\frac{-2 R}{\kappa^{2}}+g^{\mu \nu} \partial_{\mu} \phi \partial_{\nu} \phi-m^{2} \phi^{2}+g^{\mu \nu} \partial_{\mu} \Phi \partial_{\nu} \Phi-m^{2} \Phi^{2}\right)
$$

The background field method [16] ensures that order by order our weak field theory is a Lorentz invariant quantum field theory (QFT) and we can use the metric $\eta_{\mu \nu}$ to raise and lower indices. Moreover, coordinate invariance will only hold up to the order we specify, and will have the interpretation of a gauge transformation.

We follow the Misner, Thorne, and Wheeler sign convention [17] with a mostly 
minus metric

$$
\eta_{\mu \nu}=\left(\begin{array}{cccc}
1 & 0 & 0 & 0 \\
0 & -1 & 0 & 0 \\
0 & 0 & -1 & 0 \\
0 & 0 & 0 & -1
\end{array}\right) .
$$

The first step in our approach is to expand the inverse of the metric and its determinant. For $g_{\mu \nu}^{-1}$ we use

$$
g^{\alpha \mu} g_{\mu \nu}=\delta_{\nu}^{\alpha}
$$

and write down all the terms in $g^{\alpha \mu}$ that are consistent with Lorentz covariance, we choose them so that we cancel $h$ in $(1.3)$ order by order, i.e.

$$
g^{\mu \nu}=\eta^{\mu \nu}-\kappa h^{\mu \nu}+\kappa^{2}(h \cdot h)^{\mu \nu}-\kappa^{3}(h \cdot h \cdot h)^{\mu \nu}+O\left(\kappa^{4}\right),
$$

where we have used the notation $h^{\mu \alpha} h_{\alpha \nu} \equiv(h \cdot h)_{\nu}^{\mu}$ and $\operatorname{Tr}(h \cdot h)=(h \cdot h)_{\nu}^{\nu}$.

To work out the determinant we use the relation:

$$
\operatorname{det}(A)=e^{\operatorname{tr}(\log (A))}
$$

which is a corollary of Jacobi's formula

$$
\begin{aligned}
\sqrt{|g|} & =\sqrt{\left|\operatorname{det}\left(\eta_{\mu \nu}+\kappa h_{\mu \nu}\right)\right|} \stackrel{\kappa \ll 1}{\cong} \sqrt{\operatorname{det}\left(\delta_{\mu}^{\nu}+\kappa h_{\mu}^{\nu}\right)}=e^{\frac{1}{2} \operatorname{Tr}\left(\log \left(\delta_{\mu}^{\nu}+\kappa h_{\mu}^{\nu}\right)\right)} \\
& =e^{\frac{\kappa}{2}\left(h-\frac{\kappa}{2} \operatorname{Tr}(h \cdot h)\right)+O\left(\kappa^{3}\right)} \approx 1+\kappa \frac{h}{2}+\frac{\kappa^{2}}{8}\left(h^{2}-2 \operatorname{tr}(h \cdot h)\right) .
\end{aligned}
$$

After these preliminary steps we are now ready to calculate the Feynman rules for scattering amplitudes with gravitons and scalars. We will write these down later and focus here on a discussion of the quantization.

A crucial consequence of the coordinate invariance of the gravity Lagrangian is 
that perturbative quantum gravity is a gauge theory. The most general coordinate transformation that leaves the perturbation field weak is of the form

$$
\frac{\partial x^{\mu}}{\partial x^{\prime \nu}}=\delta_{\nu}^{\mu}+\kappa \partial_{\nu} \xi^{\mu}
$$

Diffeomorphism invariance imposes gauge conditions order by order in perturbation theory. At leading order we require the amplitude to be invariant under the gauge transformation:

$$
h_{\lambda \rho}^{\prime}=h_{\lambda \rho}-\partial_{\lambda} \xi_{\rho}-\partial_{\rho} \xi_{\lambda}
$$

Traditionally, it has been perceived that the main problem with perturbative quantum gravity was its non renormalizability: the divergences generated order by order by the loop integrals cannot be regularised by fixing a finite number of parameters. These divergences are regularised by introducing counter-terms, but counter-terms for divergences up to a certain order introduce divergences at higher orders [18]. Here, if we consider the theory to be valid at all energies we are forced to introduce an infinite number of counter-terms and lose all predictive power. This might be a milder problem in supergravity theories [19].

However, if we consider gravity as an effective theory we can impose an energy cut-off for the integrals and work at a fixed order in the perturbation expansion. This is due to the fact that higher order operators are suppressed by inverse powers of the cut-off, hence only a finite number of them contribute at a given order in accuracy.

The cut-off represents the scale at which our effective theory is no longer valid. We expect that this effective theory is what remains after integrating out the high energy degrees of freedom from a renormalizable theory. 


\subsection{Perturbation theory at high energies and the need for resummation}

We have seen that treating Einstein-Hilbert gravity as an effective theory allows us to have a perturbative expansion that is well behaved. However, at high center of mass energies $s$, powers of logarithms of the energy can render this expansion invalid if $\kappa \sqrt{s} \ln s \approx \mathcal{O}(1)$ and it is mandatory to work with an all order resummation. This problem has been studied in detail in Yang-Mills (YM) theories where it has been shown that these large logarithms arise from the sum of processes with inelastic multiple jet production with the outgoing jets strongly ordered in rapidity. In terms of a total cross section for the high energy scattering of two particles A and B

$$
\left.\sigma_{t o t}\left(s=e^{y_{A}-y_{B}}\right) \stackrel{s \rightarrow \infty}{=} \frac{1}{s} \sum_{n=0}^{\infty}\right|_{\mathrm{B}} ^{\mathrm{A}_{n}, k_{i \perp}}
$$

In more detail, one finds that these large logarithms $\alpha_{s}^{n} \ln ^{n}(s) \sim \alpha_{s}^{n}\left(y_{A}-y_{B}\right)^{n}$ are generated by final state configurations with strong ordering in the rapidities of the emitted particles, i.e.

$$
y_{A} \ggg y_{1} \ggg y_{2} \ggg \ldots \ggg y_{n} \ggg y_{B},
$$

and with comparable transverse momenta

$$
k_{i}^{2} \approx k^{2}
$$


This limit, which is called Multi-Regge Kinematics (MRK) since it imposes the Regge limit in every energy subchannel, allows us to resum these logarithms, since the transverse and longitudinal space decouple from each other. Schematically, we can integrate over the phase space of soft emissions where the strong ordering in rapidities generates the same power in the coupling as in the logs of energy:

$$
\begin{aligned}
\sigma_{\text {tot }}^{L L} & =\sum_{n=0}^{\infty} \mathcal{C}_{n}^{L L}\left(k_{\perp}\right) \alpha_{s}^{n} \int_{y_{B}}^{y_{A}} \mathrm{~d} y_{1} \int_{y_{B}}^{y_{1}} \mathrm{~d} y_{2} \ldots \int_{y_{B}}^{y_{n-1}} \mathrm{~d} y_{n} \\
& =\sum_{n=0}^{\infty} \frac{\mathcal{C}_{n}^{L L}\left(k_{\perp}\right)}{n !} \alpha_{s}^{n}\left(y_{A}-y_{B}\right)^{n}
\end{aligned}
$$

where $y_{A}-y_{B}$ is the rapidity difference between particles $\mathrm{A}$ and $\mathrm{B}$. Moreover, one can calculate the leading coefficients $\mathcal{C}_{n}^{L L}\left(k_{\perp}\right)$ order by order using the "leading logarithmic" (LL) Balitsky, Fadin, Lipatov, Kuraev (BFKL) formalism [20, 21, 22]. Next-to-leading corrections, which consist of energy logs with a power one unit smaller than that of the strong coupling in each Feynman diagram, are sensitive to the running of the coupling constant and any possible rescaling of the energy variable in the resummed logarithms $y_{A}-y_{B}=\ln \frac{s}{s_{0}} \rightarrow \ln \frac{s}{s_{0} e^{\mathcal{B}}}$

$$
\begin{aligned}
\sigma_{\text {tot }} & =\sum_{n=0}^{\infty} \frac{\mathcal{C}_{n}^{L L}\left(k_{i \perp}\right)}{n !}\left(\alpha_{s}-\mathcal{A} \alpha_{s}^{2}\right)^{n}\left(y_{A}-y_{B}-\mathcal{B}\right)^{n} \\
& =\sigma_{\text {tot }}^{L L}-\sum_{n=1}^{\infty} \frac{\mathcal{B C}_{n}^{L L}\left(k_{i \perp}\right)+(n-1) \mathcal{A C}_{n-1}^{L L}\left(k_{i \perp}\right)}{(n-1) !} \alpha_{s}^{n}\left(y_{A}-y_{B}\right)^{n-1}
\end{aligned}
$$

This multi-particle emission is encoded in the kernel of the BFKL equation. To calculate the kernel one can use the optical theorem to obtain an infinite tower of ladder diagrams:

$$
\sigma_{t o t}\left(s=\left.e^{y_{A}-y_{B}} \stackrel{s \rightarrow \infty}{=} \frac{1}{s} \sum_{n=0}^{\infty}\right|_{y_{n}, k_{i \perp}} ^{A}\right.
$$


A

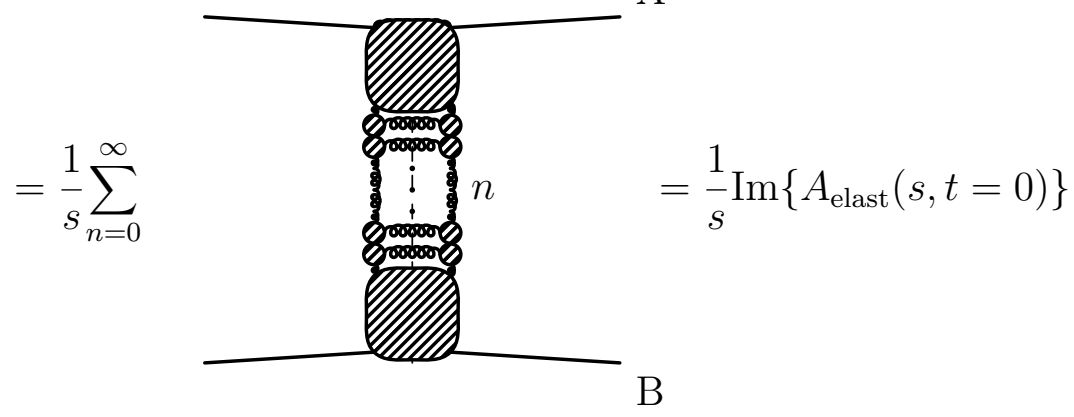

The elastic amplitudes in this kinematical limit can be interpreted as the exchange of an emergent particle, the hard "pomeron". This pomeron can be seen as a bound state of two "Reggeized" gluons in the $t$-channel.

Fig 1.1 defines our labeling convention for the rest of this thesis, where we calculate this vertex in five point amplitudes. We now express these momenta in terms of Sudakov variables, which have momentum conservation built in. We begin by defining

$$
k=k_{1}-k_{2}
$$

and expanding them in terms of

$$
k_{i}^{\mu}=\alpha_{i} p^{\mu}+\beta_{i} q^{\mu}+k_{i \perp}^{\mu}
$$

where $p, q$ are the momenta of the incoming particles (which need not be gluons), and $k_{i \perp} \cdot p=k_{i \perp} \cdot q=p^{2}=q^{2}=0$.

In Sudakov variables the MRK limit reads

$$
\begin{aligned}
& 1 \ggg \alpha_{1} \ggg \alpha_{2} \approx k_{i \perp}^{2} / s, \\
& k_{i \perp}^{2} / s \approx \beta_{1} \lll \beta_{2} \lll 1 .
\end{aligned}
$$

The absence of a hierarchy between the transverse momenta is what characterizes BFKL evolution and enables the resummation of the dominant logarithmic contributions. Lipatov's MRK effective vertex for the coupling of two Reggeized 


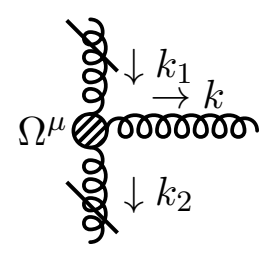

Figure 1.1: Lipatov Effective Vertex

gluons to an on-shell gluon in QCD [23, 24] will be calculated again in this thesis

$$
\Omega^{\mu}\left(k_{1}, k_{2}\right)=\left(\alpha_{1}+\frac{2 k_{i \perp}^{2}}{s \beta_{2}}\right) p^{\mu}+\left(\beta_{2}+\frac{2 k_{2 \perp}^{2}}{s \alpha_{1}}\right) q^{\mu}-\left(k_{1}+k_{2}\right)_{\perp}^{\mu}
$$

This vertex is non-local (some of the information of the amplitude is absorbed into its denominators) and gauge invariant (it obeys the Ward identity):

$$
\left(q_{1}-q_{2}\right)_{\mu} \Omega^{\mu}\left(q_{1}, q_{2}\right)=0
$$

In gravity, it is also possible to get a glimpse of the all orders amplitudes if they are evaluated in certain limits, with a remarkable example being the work of Lipatov evaluating multi-graviton scattering processes in MRK. In this region the amplitudes can be written in a factorized form which allows for the construction of a high energy effective action,where the neglected modes are those which contribute $\mathcal{O}\left(e^{-\left|y_{i}-y_{i+1}\right|}\right)$ to the scattering amplitudes, from which to generate them [25]. The key ingredients in these calculations are the reggeization of the graviton [26, 27] (which we will not discus in this thesis) together with a full control of eikonal and double logarithmic contributions [23, 28, 29] (also not discussed in this work where we focus on inelastic amplitudes at leading order in the coupling). A remarkable result in Lipatov's investigations is that the graviton emission effective vertex can be "almost" written as a double copy of the 
gluon emission effective vertex when both are evaluated in MRK and the latter is calculated in QCD [20, 21, 22]. In more detail, in a similar fashion to the QCD case, one finds an effective vertex [25, 23, 28] given by

$$
\Gamma^{\mu \nu}=\Omega^{\mu} \Omega^{\nu}-\mathcal{N}^{\mu} \mathcal{N}^{\nu}
$$

with

$$
\mathcal{N}^{\mu}\left(q_{i}, q_{i+1}\right)=-2 i \sqrt{\beta_{i} \alpha_{i+1}}\left(\frac{p_{1}^{\mu}}{\beta_{i+1}}+\frac{p_{2}^{\mu}}{\alpha_{i}}\right)
$$

where $\Omega^{\mu}$ is the effective vertex from QCD. The term $\mathcal{N}^{\mu} \mathcal{N}^{\nu}$ is interesting because it breaks the simple factorization in terms of QCD amplitudes.

High energy scattering has also been studied in string theory both in the closed string [30] and in the open string case [31]. In this latter reference the multi-Regge limit of the amplitude was also investigated.

\subsection{Color Kinematics Duality and BCJ}

The relation between scattering amplitudes in gauge and gravity theories has been the subject of extensive research in recent years (for reviews on the subject we refer the reader to [32]). The fact that in a certain sense gravity can be regarded as the "square" of a gauge theory is suggested by string theory where tree-level graviton (closed string) amplitudes can be written as linear combinations of the product of gluon (open string) amplitudes, as found by Kawai, Lewellen, and Tye (KLT) [33, 34]. In the field theory limit they translate into similar relations for the corresponding graviton and gluon amplitudes in quantum field theory [10, 11, 12]

$$
\mathcal{M}_{n}^{\text {grav }} \sim A_{i, n}^{Y M} K_{i j} A_{j, n}^{Y M}
$$


For example, at lower points these look like

$$
\begin{aligned}
\mathcal{M}_{3}^{\text {grav }} & \sim A_{3}^{Y M}(1,2,3) A_{3}^{Y M}(1,2,3), \\
\mathcal{M}_{4}^{\text {grav }} & \sim A_{4}^{Y M}(1,2,3,4) A_{4}^{Y M}(1,2,4,3), \\
\mathcal{M}_{4}^{\text {grav }}(1,2,3,4,5) & \sim s_{12} s_{34} A_{4}^{Y M}(1,2,3,4,5) \mathcal{A}_{4}^{Y M}(2,1,4,3,5) \\
& +s_{13} s_{24} A_{4}^{Y M}(1,3,2,4,5) \mathcal{A}_{4}^{Y M}(3,1,4,2,5) .
\end{aligned}
$$

Unfortunately, no similar factorization is known for string loop amplitudes, due to the coupling of left- $\mathcal{A}_{L i}$ and right- $\mathcal{A}_{R i}$ moving terms.

More recently, a surprising relation between gluon and graviton amplitudes, dubbed color-kinematics duality, has been found by Bern, Carrasco, and Johansson (BCJ) [35] (see also [36, 37, 38, 39, 40]).

This prescription allows us to obtain graviton amplitudes from gluon amplitudes of two YM theories. The first step is to write the amplitude of a YM theory $\mathcal{F}$ as a sum over all Feynman diagram topologies $\Gamma$ with only trivalent vertices:

$$
\mathcal{A}(\mathcal{F})=g^{n-2} \sum_{i \in \Gamma} \frac{c_{i} n_{i}(\mathcal{F})}{d_{i}}
$$

The denominators $d_{i}$ and color factors $c_{i}$ are given by those associated with the corresponding trivalent vertices and propagators in the Feynman rules of $\mathcal{F}$, and $g$ is the YM coupling constant. The numerators are given by the remaining factor in each term. There is some freedom in choosing these numerators because not all the color factors are independent, they are related by Jacobi identities

$$
j(c)_{\alpha}=c_{i}+c_{j} \pm c_{k}=0,
$$




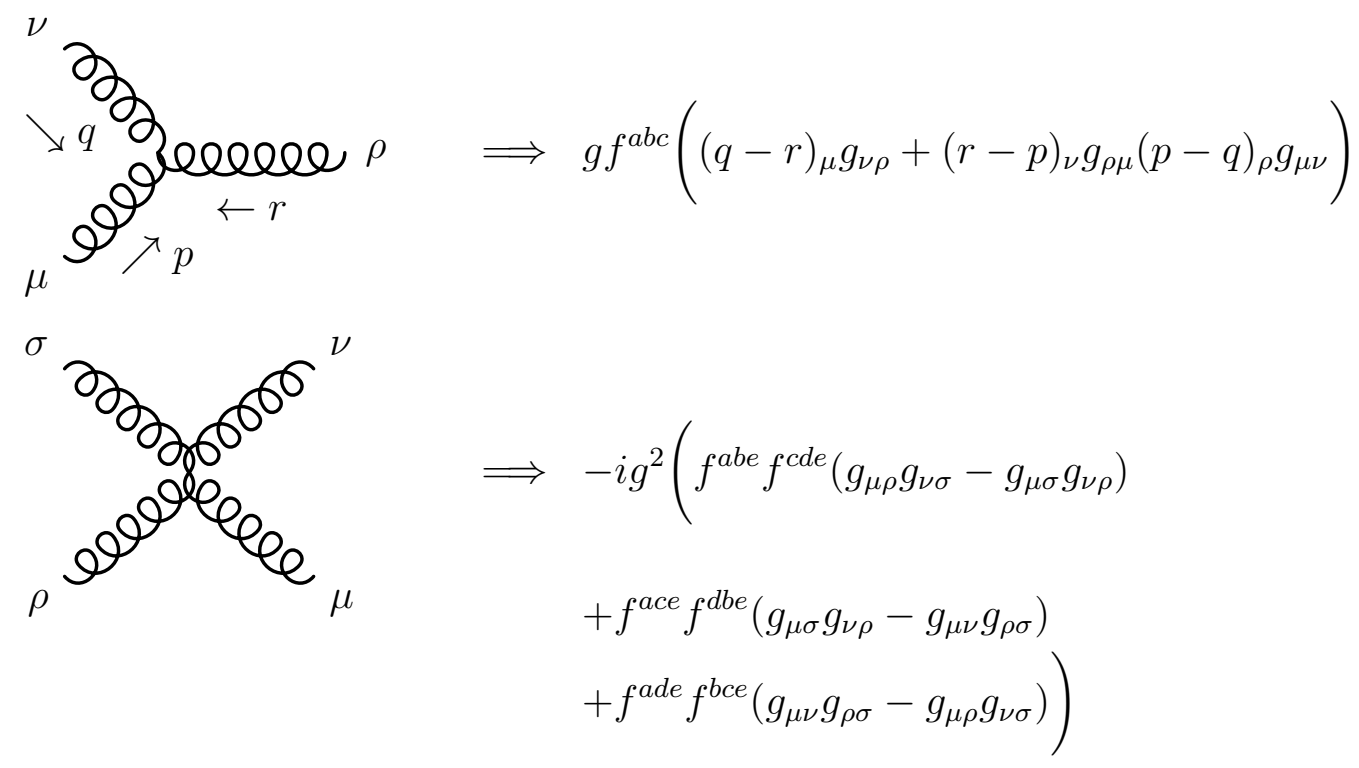

Figure 1.2: Feynman rules for the gluon vertices

which allow us to enforce analogous Jacobi identity-like equations

$$
j(n)_{\alpha}=n_{i}+n_{j} \pm n_{k}=0
$$

for the numerators. When these equations hold we obtain a graviton amplitude for a theory $\mathcal{Z}$ from two gluon amplitudes $\mathcal{A}_{n}(\mathcal{F}), \mathcal{A}_{n}(\mathcal{Y})$ in theories $\mathcal{F}, \mathcal{Y}$ with a YM sector and the same color structure using the following prescription

$$
\mathcal{M}(\mathcal{Z})=i\left(\frac{\kappa}{2}\right)^{n-2} \sum_{i \in \Gamma} \frac{n_{i}(\mathcal{Y}) n_{i}(\mathcal{F})}{d_{i}}
$$

where $\kappa=\sqrt{\frac{8 \pi G}{c^{2}}}$. To write the YM amplitude in terms of trivalent graphs (which is required by dimensional consistency) one has to rewrite the four gluon vertex as two joined trivalent vertices. This is possible in YM because, as follows from Fig. 1.2. four-gluon vertices have the color structure of two three-gluon vertices joined by a propagator $c_{4}^{a b c d} \propto \sum f^{a b i} d^{i j} f^{d e j}$ so we can recover the color and propagator 


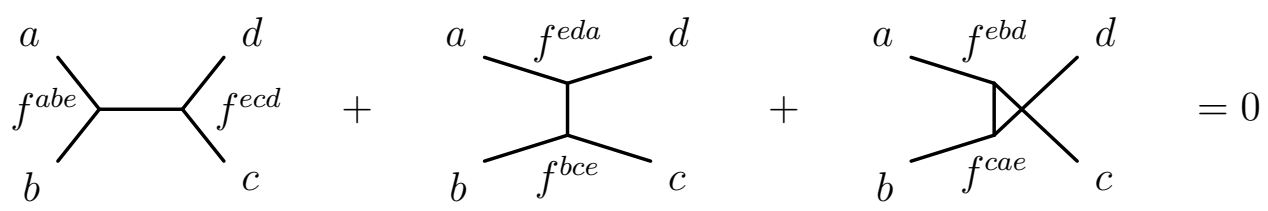

Figure 1.3: The Jacobi identity

structure of an amplitude with only trivalent graphs by multiplying and dividing by the appropriate factor ${ }^{1}$.

The overall color factors $c_{i}=\prod f^{\cdots} \ldots f^{\cdots}$ for each trivalent diagram obey Jacobi identities which they inherit from the Jacobi identity for the structure constants

$$
f^{a b i} f^{i c d}+f^{c a i} f^{i b d}+f^{b c i} f^{i a d}=0 .
$$

This expression can be understood diagrammatically by permuting the four legs that attach to every internal leg, which generate all the Jacobi identities for $c_{i}$ as can be seen in Fig. (1.3).

\footnotetext{
${ }^{1}$ That we can write YM amplitudes purely in terms of trivalent graphs can also be seen by introducing a non-dynamic antisymmetric auxiliary field that removes the 4 gluon vertex in the
} Lagrangian

$$
\mathcal{L}_{4}=-\frac{g^{2}}{4}\left(f^{a b c} A^{\mu b} A^{\nu c}\right)^{2}=-\frac{1}{2} B^{a \mu \nu} B_{\mu \nu}^{a}+i \frac{g}{\sqrt{2}} f^{a b c} B_{\mu \nu}^{a} A^{\mu b} A^{\nu c} .
$$

We can now integrate out the B-field which can in practice be done by taking its equations of motion as it has quadratic couplings at most

$$
\frac{\delta \mathcal{L}}{\delta B^{a \mu \nu}}=0=-B^{a \mu \nu}+i \frac{g}{\sqrt{2}} f^{a b c} A^{\mu b} A^{\nu c} .
$$




\subsubsection{Numerators that obey BCJ}

To solve the BCJ equations, we transform the numerators

$$
n_{i} \rightarrow n_{i}^{\prime}=n_{i}+\Delta_{i}
$$

so that they obey the Jacobi identities

$$
j(n)_{\alpha}=n_{i}^{\prime}+n_{j}^{\prime} \pm n_{k}^{\prime}=0,
$$

while leaving the YM amplitude invariant

$$
\mathcal{A}\left(n_{i}^{\prime}\right)=g^{n-2} \sum_{i \in \Gamma} \frac{c_{i} n_{i}^{\prime}}{d_{i}}=\mathcal{A}\left(n_{i}\right)
$$

For this to happen, the shifts $\Delta_{i}$ must obey the condition

$$
\sum_{i \in \Gamma} \frac{c_{i} \Delta_{i}}{d_{i}}=0
$$

Because we only allow transformations that leave the amplitude invariant 1.34 we call (1.32) "generalized gauge transformations" by analogy with gauge transformations that shift the gluon polarization vectors $\varepsilon_{\mu}(k) \rightarrow \varepsilon_{\mu}(k)+\lambda k_{\mu}$, leaving the amplitude invariant. As a matter of fact, gauge transformations are a particular case of 1.32 . The existence of these shifts is made possible precisely by the Jacobi identities, which allow the shift in one diagram to be compensated somewhere else.

The full color dressed amplitudes $\mathcal{A}$ can be written in terms of color ordered partial amplitudes $A(1, \sigma(2), \ldots, \sigma(m))$, where $m$ is the number of legs of the 
amplitude

$$
\mathcal{A}_{m}\left(n_{i}\right)=\sum_{S_{m-1}} \operatorname{Tr}\left(T^{a_{1}} T^{a_{\sigma(2)}} \ldots T^{a_{\sigma(m)}}\right) A(1, \sigma(2), \ldots, \sigma(m))\left(n_{i}\right) .
$$

Cyclicity of the trace means that we only have to sum over $(m-1)$ ! partial amplitudes. However, not all of these are independent: the Kleiss-Kuijf [41] relations bring the number of these down $\mathrm{tg}^{2}(m-2)$ !. We can recast $(1.34)$ into equivalent equations for the partial amplitudes

$$
A(1, \sigma(2), \ldots, \sigma(m))\left(n_{i}^{\prime}\right)=A(1, \sigma(2), \ldots, \sigma(m))\left(n_{i}\right) .
$$

The existence of a solution to both these set of equations 1.33 and 1.34 is non-trivial. This is because not all the equations are independent which places constraints on the partial amplitudes and implies that the size of the independent set goes down to $(m-3)$ !. It has been in fact proven that these solutions exist for tree amplitudes for any number of legs. However, no such proof exists at loop level.

Although the initial proof of the existence of solutions to the BCJ equations at tree level, using string theory monodromy arguments [42], and later proofs using field theory [43], focused on the existence of solutions, more recent proofs are more compelling as they exhibit the relation between these solutions and the double copy more clearly. In particular, a generalized set of KLT relations have been found [44] which relate closed string (gravitational) to open string (gauge) amplitudes at tree level at all points:

$$
\mathcal{M}_{m}=\frac{-i^{m-3}}{4} \sum_{\sigma} \sum_{\gamma, \beta} \mathcal{S}_{\alpha^{\prime}}[\gamma(\sigma(2), \ldots, \sigma(j-1)) \mid \sigma(2, \ldots, j-1)]_{k_{1}}
$$

\footnotetext{
2 Alternatively, one could use the color stripped coefficients of the trivalent color factors, after solving the Jacobi identities the remaining independent set has $(m-2)$ ! members.
} 


$$
\begin{aligned}
& \times \mathcal{S}_{\alpha^{\prime}}[\beta(\sigma(j), \ldots, \sigma(m-2)) \mid \sigma(j, \ldots, m-2)]_{k_{1}} \\
& \times \mathcal{A}_{m}(1, \sigma(2, \ldots, m-2), m-1, m) \\
& \times \mathcal{A}_{m}(\gamma(\sigma(2), \ldots, \sigma(j-1)), 1, m-1, \beta(\sigma(j), \ldots, \sigma(m-2))),
\end{aligned}
$$

where the "string" momentum kernel $\mathcal{S}_{\alpha^{\prime}}$ is given by

$$
\mathcal{S}_{\alpha^{\prime}}\left[i_{1}, \ldots, i_{k} \mid j_{1}, \ldots, j_{k}\right]_{p} \equiv\left(\pi \alpha^{\prime} / 2\right)^{-k} \prod_{t=1}^{k} \sin \left(\pi \alpha^{\prime}\left(p \cdot k_{i_{t}}+\sum_{q>t}^{k} \theta\left(i_{t}, i_{q}\right) k_{i_{t}} \cdot k_{i_{q}}\right)\right)
$$

Here, $\alpha^{\prime}$ is the string slope and $\theta\left(i_{t}, i_{q}\right)$ equals 1 if the ordering of the legs $i_{t}$ and $i_{q}$ is opposite in the sets $i_{1}, \ldots, i_{k}$ and $j_{1}, \ldots, j_{k}$, and 0 if the ordering is the same. To make the connection with BCJ clear, note that the sum in 1.38 runs over $(m-3)$ ! permutations as three of the legs are held fixed. This connection is made explicit by the annihilation property of the kernel

$$
\sum_{\sigma} \mathcal{S}_{\alpha^{\prime}}[\sigma(2, \ldots, m-1) \mid \beta(\sigma(2), \ldots, \sigma(m-1))]_{k_{1}} \mathcal{A}_{n}(n, \sigma(2, \ldots, m-1), 1)=0
$$

which leaves an independent set of $(m-3)$ ! partial amplitudes.

The proof of 1.38 and 1.40 has been carried out using the Britto, Cachazo, Feng, and Witten (BCFW) [45] recursion relations inductively by assuming it is true up to $m-1$ legs, making a BCFW shift taking two reference legs, and applying it to every amplitude in the identity. These identities all extend to field theory in the limit $\alpha^{\prime} \rightarrow 0$ where the kernel takes the form

$$
\mathcal{S}\left[i_{1}, \ldots, i_{k} \mid j_{1}, \ldots, j_{k}\right]_{p} \equiv \prod_{t=1}^{k}\left(s_{1 i_{t}}+\sum_{q>t}^{k} \theta\left(i_{t}, i_{q}\right) s_{i_{t} i_{q}}\right)
$$

As we have already mentioned, for loop-level amplitudes the existence of solutions to the BCJ equations remains an open problem. Solutions have been found 
for up to four loop amplitudes at the integrand level but a proof of the existence of these solutions is yet to be found. This is harder at loop level due to the difficulty of finding consistent labeling for the loop momenta between graphs that are related by their Jacobi relations.

\subsubsection{BCJ at loop level}

So far we have established the relation between BCJ and KLT, and the constraints that BCJ puts on YM amplitudes. However, it is at loop level where BCJ becomes really useful as it allows us to construct graviton integrands from YM ones, which is beyond the applicability of KLT. Furthermore, one finds that it is possible to build graviton amplitudes with varying degrees of supersymmetry by combining the numerators of different supersymmetric YM amplitudes.

For two super Yang-Mills theories $\mathcal{F}, \mathcal{Y}$ with number of supersymmetries $\mathcal{N}=$ $f$ and $\mathcal{N}=y$ respectively, $\mathcal{M}(\mathcal{F}, \mathcal{Y}, n)$ is an $n$-point amplitude in $\mathcal{N}=f+y$ supergravity (SUGRA)

$$
\mathcal{M}(\mathcal{N}=f+y, n)=g^{n-2} \sum_{i \in \Gamma} \frac{n_{i}(\mathcal{N}=f) n_{i}(\mathcal{N}=y)}{d_{i}}
$$

if at least one set of numerators obey the BCJ equations (both sets must obey (1.34), but due to this last property, only one set has to obey (1.33)). This is plausible by counting the physical states of the theories involved, and has been explicitly confirmed for $f=4, y=0,1,2,3,4$ resulting in $\mathcal{N}=4,5,6,7,8$ SUGRA amplitudes respectively [46]. We should point out that both $f, y$ can be zero. In this case the gravity theory is the field theory limit of the bosonic string with a graviton, an anti-symmetric tensor and a dilaton. So far solutions for BCJ numerators have been found at loop level for up to three loops.

Although a full proof for BCJ at loop level remains elusive, one can remarkably 
prove that if one can find a solution for the BCJ equations at loop level, the double copy amplitude is a gravity amplitude. More importantly this proof works at the integrand level, proceeds by induction and uses generalized unitarity [47] to factor an $n$ point gravity into products of lower multiplicity gravity amplitudes (explicit proofs for $m$ point amplitudes at one and two loops have been constructed [48, [49, 50]). 


\section{Chapter 2}

\section{Perturbative Einstein-Hilbert \\ Coupled to Scalars and the Regge Limit}

\subsection{Constructing Effective Vertices}

In the present chapter we take a bottom-up approach and evaluate inelastic amplitudes at tree level both in Einstein-Hilbert gravity and QCD. We obtain expressions valid in general kinematics and then go to MRK to reproduce Lipatov's results both in gravity and QCD. Our computations are performed using conventional Feynman rules, with the aid of computer algebra [51] in the much more complicated case of gravity.

In Section 2.3 a five-point amplitude is evaluated in QCD with two pairs of distinct quarks plus an emitted gluon. We split the contribution of the threegluon vertex into two pieces which, when added to the diagrams corresponding to gluon emission from the same fermion line, generate an effective vertex which 
is gauge invariant. In this way the amplitude can be simply decomposed into the sum of two topologies constructed with this effective vertex. The MRK limit of this sum coincides with Lipatov's gluon emission vertex. In Section (2.5) a similar calculation is performed in the case of Einstein-Hilbert gravity, where now the five-point amplitude consists of two pairs of distinguishable scalars and one emitted graviton. Operating in de Donder gauge we write the result for the exact amplitude in a Sudakov decomposition of the participating momenta. Similarly to the QCD case, a new effective vertex appears which allows to write the amplitude as the sum of two gauge invariant topologies written in terms of it. This simplification occurs only after noticing a novel and subtle partial cancellation between the two diagrams containing the two-scalar - two-graviton vertex, and splitting the three-graviton vertex into two different pieces. When taking the MRK limit of the sum of these two new effective diagrams we recover Lipatov's results for the graviton emission vertex [23, 28, 29]. We take then the MRK limit of our exact calculation to check that it can indeed be written as the square of the QCD gluon emission vertex previously calculated, plus a contribution needed to keep consistency with the Steinmann relations. This structure is deeply connected to the proposal that gravity can be understood as a "double copy" of a gauge theory (see e.g. [52] for a review on the subject).

\subsection{Feynman rules for perturbative quantum grav- ity}

In Chapter 1 we discussed how to make a perturbative expansion for quantum gravity, here we will introduce the calculation of the Feynman rules we use to calculate our amplitudes. Let us remark that we wil derive what, as far as we 
know, is the simplest form for the three-graviton vertex. To write the scalargraviton vertices we expand:

$$
\begin{aligned}
\sqrt{|g|} g^{\mu \nu} & =\left(1+\kappa \frac{h}{2}+\frac{\kappa^{2}}{8}\left(h^{2}-2 \operatorname{Tr}(h \cdot h)\right)\right)\left(\eta^{\mu \nu}-\kappa h^{\mu \nu}+\kappa^{2}(h \cdot h)^{\mu \nu}\right)+O\left(\kappa^{3}\right) \\
& =\eta^{\mu \nu}+\kappa\left(\eta^{\mu \nu} \frac{h}{2}-h^{\mu \nu}\right) \\
& +\frac{\kappa^{2}}{2}\left(2(h \cdot h)^{\mu \nu}-h h^{\mu \nu}+\eta^{\mu \nu}\left(\frac{h^{2}}{4}-\frac{1}{2} \operatorname{Tr}(h \cdot h)\right)\right)+O\left(\kappa^{3}\right)
\end{aligned}
$$

from which we can obtain the two-scalar - one-graviton contribution

$$
\frac{1}{2} \kappa\left(\left(\eta^{\mu \nu} \frac{h}{2}-h^{\mu \nu}\right) \partial_{\mu} \phi \partial_{\nu} \phi-m^{2} \phi^{2} \frac{h}{2}\right)
$$

with its corresponding Feynman rule

$$
\searrow_{\nearrow_{p}}^{\sim \sim} \sim \alpha \beta \Longrightarrow-\frac{i \kappa}{2}\left(p_{\mu} q_{\nu}+p_{\nu} q_{\mu}\right)\left(-\eta^{\mu \alpha} \eta^{\nu \beta}+\frac{1}{2} \eta^{\mu \nu} \eta^{\alpha \beta}\right) .
$$

Similarly the two-scalar - two-graviton term gives

$$
\frac{1}{4} \kappa^{2}\left(\left(2(h \cdot h)^{\mu \nu}-h h^{\mu \nu}+\eta^{\mu \nu}\left(\frac{h^{2}}{4}-\frac{1}{2} \operatorname{Tr}(h \cdot h)\right)\right) \partial_{\mu} \phi \partial_{\nu} \phi\right)
$$

whose vertex is

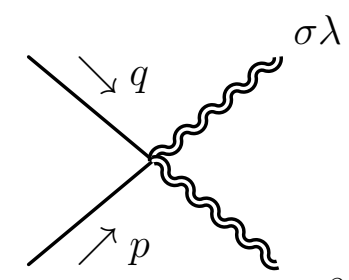

$$
\Longrightarrow-\frac{i \kappa^{2}}{4}\left(p_{\mu} q_{\nu}+p_{\nu} q_{\mu}\right)\left(\mathcal{I}_{\rho \zeta}^{\alpha \beta} \mathcal{I}_{\delta \gamma}^{\sigma \lambda}+\mathcal{I}_{\delta \gamma}^{\alpha \beta} \mathcal{I}_{\rho \zeta}^{\sigma \lambda}\right)
$$

$$
\times\left[2 \eta^{\gamma \nu} \eta^{\zeta \delta} \eta^{\mu \rho}-\eta^{\gamma \nu} \eta^{\mu \delta} \eta^{\rho \zeta}+\eta^{\mu \nu}\left(-\frac{1}{2} \eta^{\zeta \gamma} \eta^{\rho \delta}+\frac{1}{4} \eta^{\delta \gamma} \eta^{\rho \zeta}\right)\right]
$$


where we have introduced the symmetrizer

$$
\mathcal{I}_{\alpha \beta ; \gamma \delta}=\frac{1}{2}\left(\eta_{\alpha \gamma} \eta_{\beta \delta}+\eta_{\alpha \delta} \eta_{\beta \gamma}\right)
$$

The scalar propagator is just given by

$$
\Longrightarrow \frac{i}{p^{2}+i \epsilon}
$$

For the graviton propagator and three-graviton vertex we use the following identity $([53])$ :

$$
\sqrt{|g|} R[g]=\sqrt{|g|} g^{\mu \nu}\left(\Gamma_{\mu \beta}^{\alpha} \Gamma_{\nu \alpha}^{\beta}-\Gamma_{\mu \nu}^{\alpha} \Gamma_{\alpha \beta}^{\beta}\right)+\text { total derivatives }
$$

where

$$
\Gamma_{\mu \nu}^{\lambda}=\frac{1}{2} g^{\lambda \rho}\left(\partial_{\nu} g_{\rho \mu}+\partial_{\mu} g_{\rho \nu}-\partial_{\rho} g_{\mu \nu}\right)
$$

We use the canonical de Donder or harmonic gauge fixing condition

$$
F^{\lambda}(h)=\partial_{\alpha} h^{\alpha \lambda}-\frac{\partial^{\lambda} h}{2}=0
$$

which is covariant and among its nice properties has that ghosts in the FaddeevPopov formalism do not feature at tree level due to its linearity. Using this condition and integration by parts repeatedly we get the following expression for the quadratic part

$$
\mathcal{L}_{\text {grav }, \text { free }, \mathrm{gf}}=\frac{1}{2} h_{\mu \nu} \frac{-1}{4}\left(\eta^{\mu \alpha} \eta^{\nu \beta}+\eta^{\nu \alpha} \eta^{\mu \beta}-\eta^{\mu \nu} \eta^{\alpha \beta}\right) \partial^{2} h_{\alpha \beta}
$$




$$
=\frac{1}{2} h_{\mu \nu} \frac{-1}{4} \mathfrak{D}^{\mu \nu ; \alpha \beta} \partial^{2} h_{\alpha \beta} .
$$

To obtain the propagator we have to invert this, using $\mathfrak{D}_{\mu \nu ; \alpha \beta}^{-1}=2 \mathfrak{D}_{\mu \nu ; \alpha \beta}$

$$
\alpha \beta \approx \approx \sim \widetilde{\Omega} \approx \sigma \lambda \Longrightarrow \frac{i}{p^{2}+i \epsilon}\left(\eta_{\alpha \sigma} \eta_{\beta \lambda}+\eta_{\alpha \lambda} \eta_{\beta \sigma}-\eta_{\alpha \beta} \eta_{\sigma \lambda}\right) .
$$

For the three-graviton vertex we obtain eight combinatorially distinct terms by repeatedly using the de Donder condition and integration by parts:

$$
\begin{aligned}
\left.\sqrt{|g|} R[g]\right|_{3 g \text { vertex }} & =\frac{-1}{4} h^{a b} \partial_{a} h^{c d} \partial_{b} h_{c d}+\frac{1}{8} h^{a}{ }_{a} \partial_{b} h^{d e} \partial^{b} h_{d e} \\
& +\frac{-1}{2} h^{a b} \partial_{b} h_{a}{ }^{c} \partial_{c} h^{d}{ }_{d}+\frac{-1}{2} h^{a b} \eta^{c d} \partial_{c} h_{a}{ }^{e} \partial_{d} h_{b e} \\
& +\frac{1}{4} h^{a b} \eta^{c d} \partial_{c} h_{a b} \partial_{d} h^{e}{ }_{e}+h^{a b} \partial_{b} h_{c d} \partial^{d} h_{a}{ }^{c} \\
& +\frac{1}{2} h^{a b} \partial_{c} h_{b d} \partial^{d} h_{a}{ }^{c}+\frac{-1}{4} h_{a}^{a} \partial_{c} h_{b d} \partial^{d} h^{b c} .
\end{aligned}
$$

This is an important result because as far as we know, it is the simplest form for this vertex in the literature (compare, e.g., with [54] where it is claimed that eleven is probably the smallest number of terms in this gauge).

$$
\begin{aligned}
& \mu \nu \\
& \text { ح } \\
& \begin{aligned}
\Longrightarrow & i \kappa\left(p_{\sigma} q_{\lambda} \mathcal{I}^{\mu \nu}{ }_{\zeta \xi} \mathcal{I}^{\gamma \delta}{ }_{\rho \chi} \mathcal{I}^{\alpha \beta}{ }_{\tau v}+p_{\lambda} q_{\sigma} \mathcal{I}^{\gamma \delta}{ }_{\zeta \xi} \mathcal{I}^{\mu \nu}{ }_{\rho \chi} \mathcal{I}^{\alpha \beta}{ }_{\tau v}\right. \\
& +k_{\sigma} q_{\lambda} \mathcal{I}^{\mu \nu}{ }_{\zeta \xi} \mathcal{I}^{\alpha \beta}{ }_{\rho \chi} \mathcal{I}^{\gamma \delta}{ }_{\tau v}+k_{\lambda} q_{\sigma} \mathcal{I}^{\alpha \beta}{ }_{\zeta \xi} \mathcal{I}^{\mu \nu}{ }_{\rho \chi} \mathcal{I}^{\gamma \delta}{ }_{\tau v} \\
& \left.+k_{\sigma} p_{\lambda} \mathcal{I}^{\gamma \delta}{ }_{\zeta \xi} \mathcal{I}^{\alpha \beta}{ }_{\rho \chi} \mathcal{I}^{\mu \nu}{ }_{\tau v}+k_{\lambda} p_{\sigma} \mathcal{I}^{\alpha \beta}{ }_{\zeta \xi} \mathcal{I}^{\gamma \delta}{ }_{\rho \chi} \mathcal{I}^{\mu \nu}{ }_{\tau v}\right) \\
& \times \mathscr{G}^{\lambda ; \zeta \xi ; \sigma ; \rho \chi ; \tau v},
\end{aligned}
\end{aligned}
$$


where the last factor, given by

$$
\begin{aligned}
\mathscr{G}^{\lambda ; \zeta \xi ; \sigma ; \rho \chi ; \tau v} & =-\frac{1}{2} \eta^{\zeta \tau} \eta^{\lambda \chi} \eta^{\xi \sigma} \eta^{\rho \nu}-\frac{1}{4} \eta^{\zeta \tau} \eta^{\lambda \sigma} \eta^{\xi \nu} \eta^{\rho \chi}+\frac{1}{4} \eta^{\zeta \sigma} \eta^{\lambda \chi} \eta^{\xi \rho} \eta^{\tau \nu}-\frac{1}{8} \eta^{\zeta \rho} \eta^{\lambda \sigma} \eta^{\xi \chi} \eta^{\tau \nu} \\
& -\eta^{\zeta \chi} \eta^{\lambda \rho} \eta^{\xi \nu} \eta^{\tau \sigma}+\frac{1}{2} \eta^{\zeta \tau} \eta^{\lambda \sigma} \eta^{\xi \rho} \eta^{\chi \nu}+\frac{1}{4} \eta^{\zeta \rho} \eta^{\lambda \nu} \eta^{\tau \sigma} \eta^{\chi \xi}+\frac{1}{2} \eta^{\zeta \xi} \eta^{\lambda \rho} \eta^{\sigma \nu} \eta^{\chi \tau}
\end{aligned}
$$

captures all the physical information of the eight terms in the vertex whereas the remaining terms just perform symmetrizations. When the vertex is fully expanded it contains $3 ! \times 2^{3} \times 8=384$ terms.

\subsection{Quark-quark scattering with gluon emission}

Before we write down the calculations for gravity we will start with a QCD calculation for comparison. We start by describing in this section the scattering at tree level of two distinct quarks with the emission of a gluon in the final state. Our notation for the different momenta reads

$$
Q(p, j)+Q^{\prime}(q, n) \longrightarrow Q\left(p^{\prime}, i\right)+Q^{\prime}\left(q^{\prime}, m\right)+g(k, a)
$$

where $j, n, i, m$ denote the (fundamental representation) gauge indices of the incoming and outgoing quarks and $a$ the index of the outgoing gluon. At leading order in the strong coupling constant, $g$, five diagrams contribute to the total amplitude:

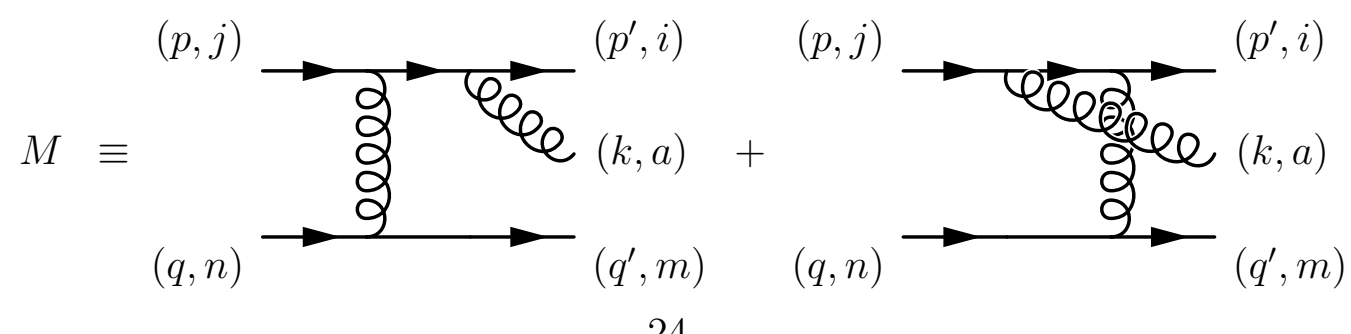



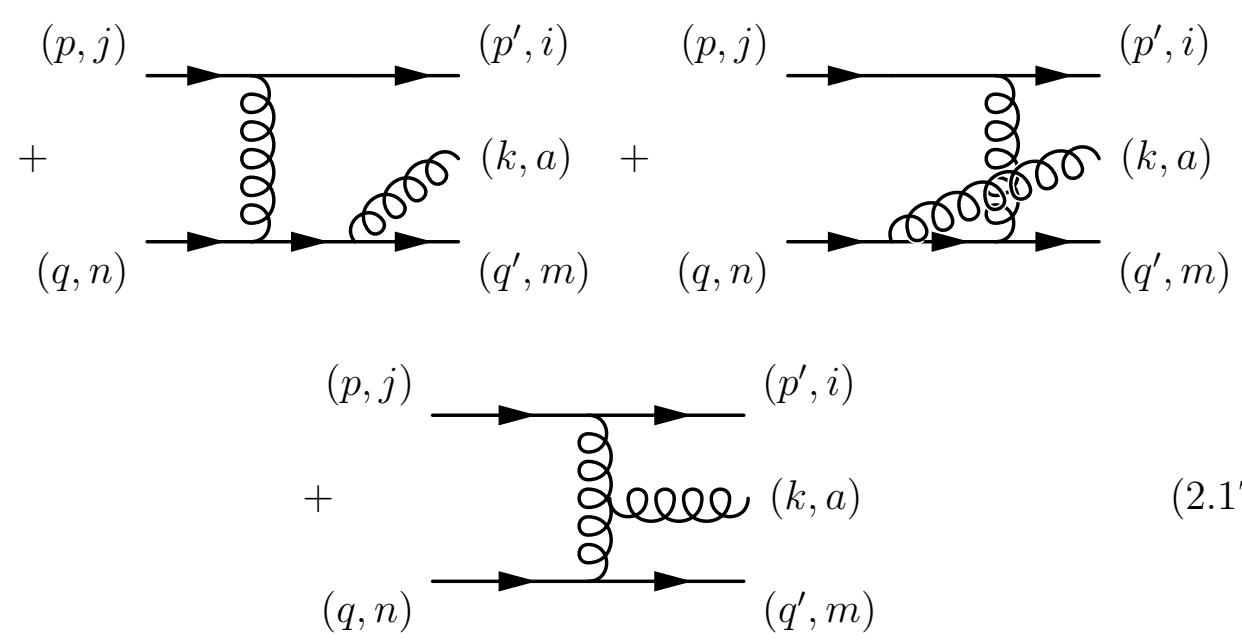

whose respective contributions we denote by

$$
M=M_{1}+M_{2}+M_{3}+M_{4}+M_{5} .
$$

The evaluation of the individual diagrams gives the result [55]

$$
\begin{aligned}
M_{1} & =-\frac{g^{3}}{2 t^{\prime}} \bar{u}\left(p^{\prime}\right) \notin T_{i k}^{a} \frac{\not p^{\prime}+\not k}{p^{\prime} \cdot k} \gamma^{\mu} T_{k j}^{b} u(p) \bar{u}\left(q^{\prime}\right) \gamma_{\mu} T_{m n}^{b} u(q), \\
M_{2} & =\frac{g^{3}}{2 t^{\prime}} \bar{u}\left(p^{\prime}\right) \gamma^{\mu} T_{i k}^{b} \frac{\not p-\not k}{p \cdot k} \notin T_{k j}^{a} u(p) \bar{u}\left(q^{\prime}\right) \gamma_{\mu} T_{m n}^{b} u(q), \\
M_{3} & =-\frac{g^{3}}{2 t} \bar{u}\left(p^{\prime}\right) \gamma^{\mu} T_{i j}^{b} u(p) \bar{u}\left(q^{\prime}\right) \notin T_{m k}^{a} \frac{\not q^{\prime}+\not k}{q^{\prime} \cdot k} \gamma_{\mu} T_{k n}^{b} u(q), \\
M_{4} & =\frac{g^{3}}{2 t} \bar{u}\left(p^{\prime}\right) \gamma^{\mu} T_{i j}^{b} u(p) \bar{u}\left(q^{\prime}\right) \gamma_{\mu} T_{m k}^{b} \frac{\not q-\not k}{q \cdot k} \notin T_{k n}^{a} u(q), \\
M_{5} & =-\frac{i g^{3}}{t t^{\prime}} \bar{u}\left(p^{\prime}\right) \gamma^{\mu} T_{i j}^{c} u(p) \bar{u}\left(q^{\prime}\right) \gamma^{\nu} T_{m n}^{b} u(q) f^{c b a} \\
& \times\left[\left(p-p^{\prime}-q+q^{\prime}\right) \cdot \varepsilon \eta_{\mu \nu}+\left(q-q^{\prime}+k\right)_{\mu} \varepsilon_{\nu}+\left(p^{\prime}-k-p\right)_{\nu} \varepsilon_{\mu}\right],
\end{aligned}
$$

where

$$
t=\left(p-p^{\prime}\right)^{2}, \quad t^{\prime}=\left(q-q^{\prime}\right)^{2}
$$


Following [56], we decompose the amplitude into two sets

$$
M_{\uparrow}=M_{1}+M_{2}+M_{5}^{\prime}, \quad M_{\downarrow}=M_{3}+M_{4}+M_{5}^{\prime \prime},
$$

where

$$
M_{5}^{\prime}=\frac{t}{t-t^{\prime}} M_{5}, \quad M_{5}^{\prime \prime}=\frac{t^{\prime}}{t^{\prime}-t} M_{5}
$$

The full amplitude is then written as

$$
M=M_{\uparrow}+M_{\downarrow} .
$$

What makes this decomposition interesting is that $M_{\uparrow}$ and $M_{\downarrow}$ are gauge invariant separately. Indeed, replacing the external polarization $\epsilon_{\mu}(k)$ by $k_{\mu}$ we find that, after some algebra,

$$
M_{1}+M_{2} \longrightarrow-\frac{i g^{3}}{t^{\prime}} f^{a b c} T_{i j}^{c} T_{m n}^{b} \bar{u}\left(p^{\prime}\right) \gamma^{\mu} u(p) \bar{u}\left(q^{\prime}\right) \gamma_{\mu} u(q)
$$

whereas

$$
M_{5}^{\prime} \longrightarrow \frac{i g^{3}}{t^{\prime}\left(t-t^{\prime}\right)} k \cdot\left(p-p^{\prime}-q+q^{\prime}\right) f^{a b c} T_{i j}^{c} T_{m n}^{b} \bar{u}\left(p^{\prime}\right) \gamma^{\mu} u(p) \bar{u}\left(q^{\prime}\right) \gamma_{\mu} u(q)
$$

When momentum conservation is imposed this last term cancels Eq. 2.28 and leads to the partial Ward identity

$$
k_{\mu} M_{\uparrow}^{\mu}=0,
$$

where the notation $M_{\uparrow} \equiv \varepsilon_{\mu}(k) M_{\uparrow}^{\mu}$ has been used. A similar treatment of the 
second combination of diagrams generates the Ward identity

$$
k_{\mu} M_{\downarrow}^{\mu}=0 .
$$

The main advantage of combining Feynman diagrams into these gauge invariant contributions is that it allows to fix the polarization of the emitted gluons independently for each of them, reducing the number of cross terms when evaluating higher order amplitudes by means of unitarity relations. In the present work the existence of these two gauge invariant combinations offers the possibility to define effective vertices which can, eventually, be obtained from a suitable effective action valid for general kinematics. In order to extract these vertices from our representation of the amplitude we write the two subamplitudes as

$$
\begin{aligned}
& M_{\uparrow}=\left[\varepsilon_{\nu}(k) \bar{u}\left(p^{\prime}\right) \Lambda_{i j ; c, a}^{\mu, \nu}\left(p, p^{\prime}, k\right) u(p)\right] \frac{-i \eta_{\mu \sigma} \delta_{c b}}{t^{\prime}}\left[\bar{u}\left(q^{\prime}\right) g \gamma^{\sigma} T_{m n}^{b} u(q)\right], \\
& M_{\downarrow}=\left[\bar{u}\left(p^{\prime}\right) g \gamma^{\mu} T_{i j}^{c} u(p)\right] \frac{-i \eta_{\mu \sigma} \delta_{c b}}{t}\left[\varepsilon_{\nu}(k) \bar{u}\left(q^{\prime}\right) \Lambda_{i j ; b, a}^{\sigma, \nu}\left(q, q^{\prime}, k\right) u(q)\right],
\end{aligned}
$$

where $\Lambda_{i j ; a, b}^{\mu, \nu}\left(p, p^{\prime}, k\right)$ is given by

$$
\begin{aligned}
& \Lambda_{i j ; d, a}^{\mu, \nu}=-\frac{i g^{2}}{2} T_{i k}^{a} T_{k j}^{d} \gamma^{\nu} \frac{\not p^{\prime}+\not k}{p^{\prime} \cdot k} \gamma^{\mu}+\frac{i g^{2}}{2} T_{i k}^{d} T_{k j}^{a} \gamma^{\mu} \frac{\not p-\not k}{p \cdot k} \gamma^{\nu} \\
& -\frac{g^{2}}{2 k \cdot\left(p-p^{\prime}\right)} f^{a d c} T_{i j}^{c} \gamma^{\alpha}\left[\left(2 p-2 p^{\prime}-k\right)^{\nu} \delta_{\alpha}^{\mu}+\left(p^{\prime}-p+2 k\right)_{\alpha} \eta^{\mu \nu}+\left(p^{\prime}-p-k\right)^{\mu} \delta_{\alpha}^{\nu}\right] .
\end{aligned}
$$

By construction it satisfies the gauge Ward identity

$$
k_{\nu} \Lambda_{i j ; a, b}^{\mu, \nu}\left(p, p^{\prime}, k\right)=0
$$

In writing this effective vertex a notation stressing the nonequivalence of the indices has been used. Its non-locality is manifest by the presence of momenta in 
the denominators. Diagrammatically, we represent it by

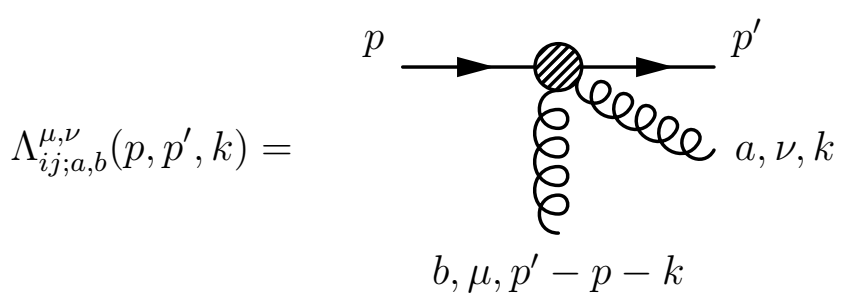

where it should be kept in mind that, by definition, three of the four legs in the vertex (those labeled by $p, p^{\prime}$ and $k$ ) are on-shell, $p^{2}=p^{\prime 2}=k^{2}=0$. The process $Q Q^{\prime} \rightarrow Q Q^{\prime} g$ at tree level can thus be represented in terms of just two Feynman diagrams, each containing a single effective vertex, i.e.

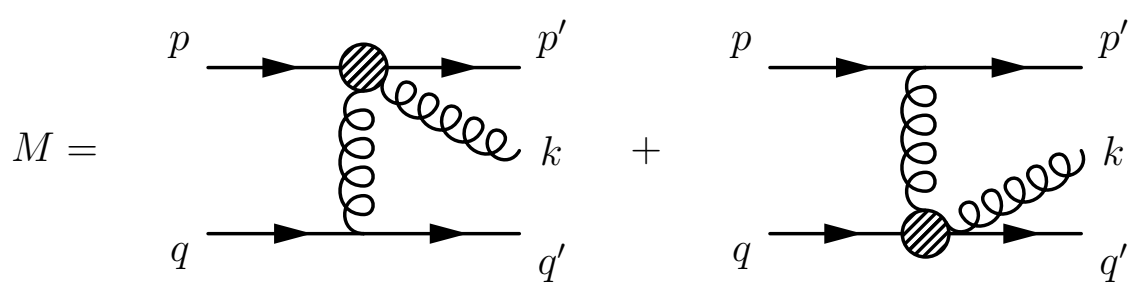

This decomposition of the amplitude in terms of two topologies with a single non-local effective vertex is exact, i.e. independent of the particular kinematical regime considered.

\subsection{Multi-Regge kinematics}

The expression for our effective vertex simplifies when restricting the amplitudes to MRK, where the limit $s=(p+q)^{2} \rightarrow \infty$ is taken while the momentum transfers $t$ and $t^{\prime}$ are kept fixed, i.e. not growing with energy. These conditions translate 
into the constraints

$$
s \gg s_{p^{\prime} k}, s_{q^{\prime} k} \gg t \sim t^{\prime}
$$

where $s_{p^{\prime} k}=\left(p^{\prime}+k\right)^{2}$ and $s_{q^{\prime} k}=\left(q^{\prime}+k\right)^{2}$. This generalizes the standard Regge limit in four-point amplitudes to the case of five-point amplitudes.

As we have already discussed in the introduction to this thesis, it is convenient to introduce the momenta $k_{1}$ and $k_{2}$ to write

$$
p^{\prime}=p-k_{1}, \quad q^{\prime}=q+k_{2}, \quad k=k_{1}-k_{2}
$$

and express them using the Sudakov parametrization of the form

$$
k_{1}^{\mu}=\alpha_{1} p^{\mu}+\beta_{1} q^{\mu}+k_{1, \perp}^{\mu}, \quad k_{2}^{\mu}=\alpha_{2} p^{\mu}+\beta_{2} q^{\mu}+k_{2, \perp}^{\mu},
$$

where $p^{2}=q^{2}=p \cdot k_{i, \perp}=0$. In terms of the Sudakov parameters $\alpha_{1,2}$ and $\beta_{1,2}$ the multi-Regge limit reads

$$
1 \gg \alpha_{1} \gg \alpha_{2}=\frac{-t^{\prime}}{s}, \quad 1 \gg\left|\beta_{2}\right| \gg\left|\beta_{1}\right|=\frac{-t}{s},
$$

which, for the emitted gluon, implies that

$$
k^{\mu} \simeq \frac{s_{q^{\prime} k}}{s} p_{1}^{\mu}+\frac{s_{p^{\prime} k}}{s} p_{2}^{\mu}+k_{\perp}^{\mu}, \quad k^{2} \simeq \frac{s_{p^{\prime} k} s_{q^{\prime} k}}{s}+k_{\perp}^{2}=0 .
$$

An alternative way of looking at the limit 2.41 is to consider that it is controlled by a counting parameter $\delta$. This is because it should be understood 
that ratios of neighboring Sudakov variables are of the same order

$$
\frac{\alpha_{2}}{\alpha_{1}}=\mathcal{O}(\delta)=\frac{\beta_{1}}{\beta_{2}}
$$

This can be achieved by rescaling the Sudakov parameters

$$
\begin{aligned}
& \alpha_{i} \longrightarrow \delta^{i} \alpha_{i}, \\
& \beta_{i} \longrightarrow \delta^{3-i} \beta_{i} .
\end{aligned}
$$

Under this rescaling, MRK is given by expanding in powers of $\delta$ and keeping the lowest term.

The MRK limit, taken on the different contributions to the total amplitude, gives the following expression for the effective vertex in Eq. 2.36):

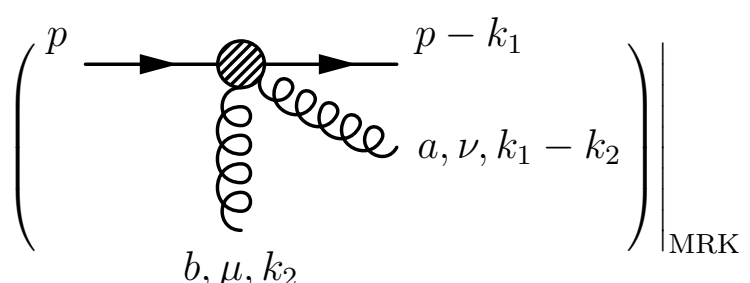

$$
\begin{aligned}
& =\frac{2 g^{2}}{t-t^{\prime}} f^{a b c} T_{i j}^{c} p^{\mu}\left[\left(\alpha_{1}-2 \frac{t-t^{\prime}}{s \beta_{2}}\right) p^{\nu}+\beta_{2} q^{\nu}-\left(k_{1}+k_{2}\right)_{\perp}^{\nu}\right] .
\end{aligned}
$$

According to Eqs. 2.32 and (2.33), to use the effective vertex in the second diagram in 2.37) one needs to replace $p^{\mu} \rightarrow q^{\mu}$ and $p^{\prime \mu} \rightarrow q^{\prime \mu}$, with $k^{\mu}$ unchanged. In terms of the momenta and Sudakov variables appearing in the MRK vertex 2.46), this amounts to

$$
p^{\mu} \longleftrightarrow q^{\mu}, \quad \alpha_{1} \longleftrightarrow-\beta_{2}, \quad k_{1 \perp}^{\mu} \longleftrightarrow-k_{2 \perp}^{\mu} .
$$


To recover Lipatov's Reggeized gluon - Reggeized gluon - gluon effective vertex at leading order in MRK we simply add the contributions of $M_{\uparrow}$ and $M_{\downarrow}$ in this limit. Diagrammatically

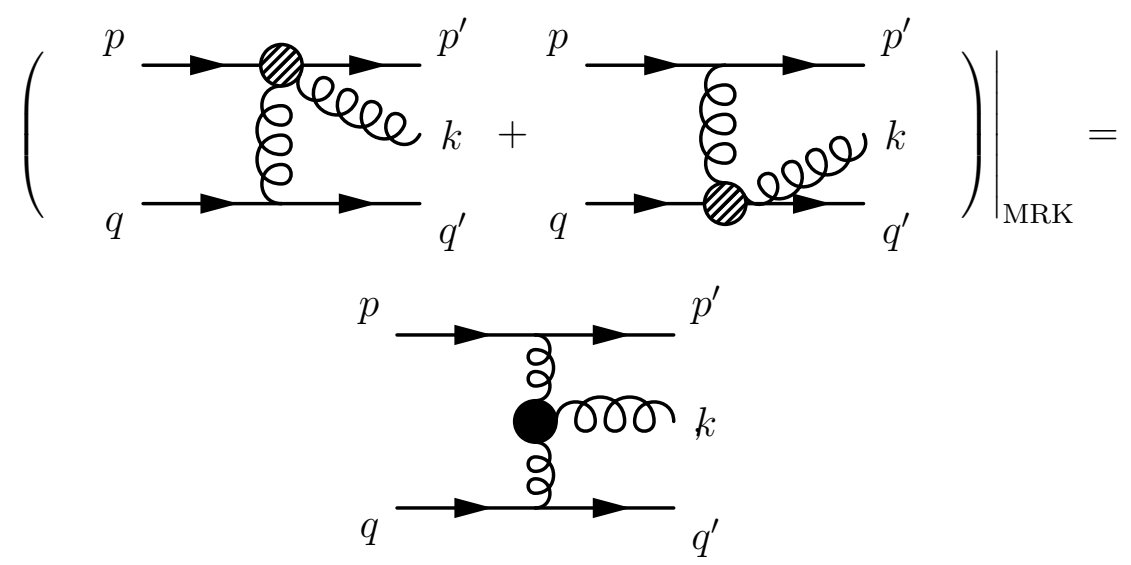

where the last effective Feynman diagram is given by

$$
M=\varepsilon_{\nu}\left(k_{1}-k_{2}\right)\left(2 g p^{\mu} T_{i j}^{c}\right)\left(\frac{-i}{t}\right) f^{c a b} \Gamma_{\mu \sigma}^{\nu}\left(\frac{-i}{t^{\prime}}\right)\left(2 g q^{\sigma} T_{m n}^{b}\right)
$$

with

$$
\Gamma_{\mu \sigma}^{\nu}=i g \eta_{\mu \sigma}\left[\left(\alpha_{1}-\frac{2 t}{s \beta_{2}}\right) p^{\nu}+\left(\beta_{2}-\frac{2 t^{\prime}}{s \alpha_{1}}\right) q^{\nu}-\left(k_{1}+k_{2}\right)_{\perp}^{\nu}\right] .
$$

This effective vertex is universal in the sense that it is independent of the nature of the external particles to which it couples. We have chosen two distinct quarks for simplicity, but it would be the same for only external gluons, for example. We follow a similar logic for the gravitational Einstein-Hilbert theory in the following section. 


\subsection{Scalar-scalar scattering with graviton emis- sion}

To minimize the number of contributing Feynman diagrams, in this section we analyze the gravitational scattering of two distinct scalars $\Phi, \Phi^{\prime}$ with the emission of a graviton in the final state with polarization $\epsilon_{\mu \nu}(k)$ :

$$
\Phi(p)+\Phi^{\prime}(q) \longrightarrow \Phi\left(p^{\prime}\right)+\Phi^{\prime}\left(q^{\prime}\right)+G(k, \epsilon)
$$

We proceed with the calculation of the corresponding amplitude using algebraic codes [51] when expressions are lengthy. A novel exact effective vertex will be obtained whose MRK limit will coincide with the one calculated by Lipatov in his works on gravity. Our exact vertex for graviton emission depends on the particular choice of external particles while its MRK limit is universal. The treelevel amplitude involves the computation of seven Feynman diagrams:

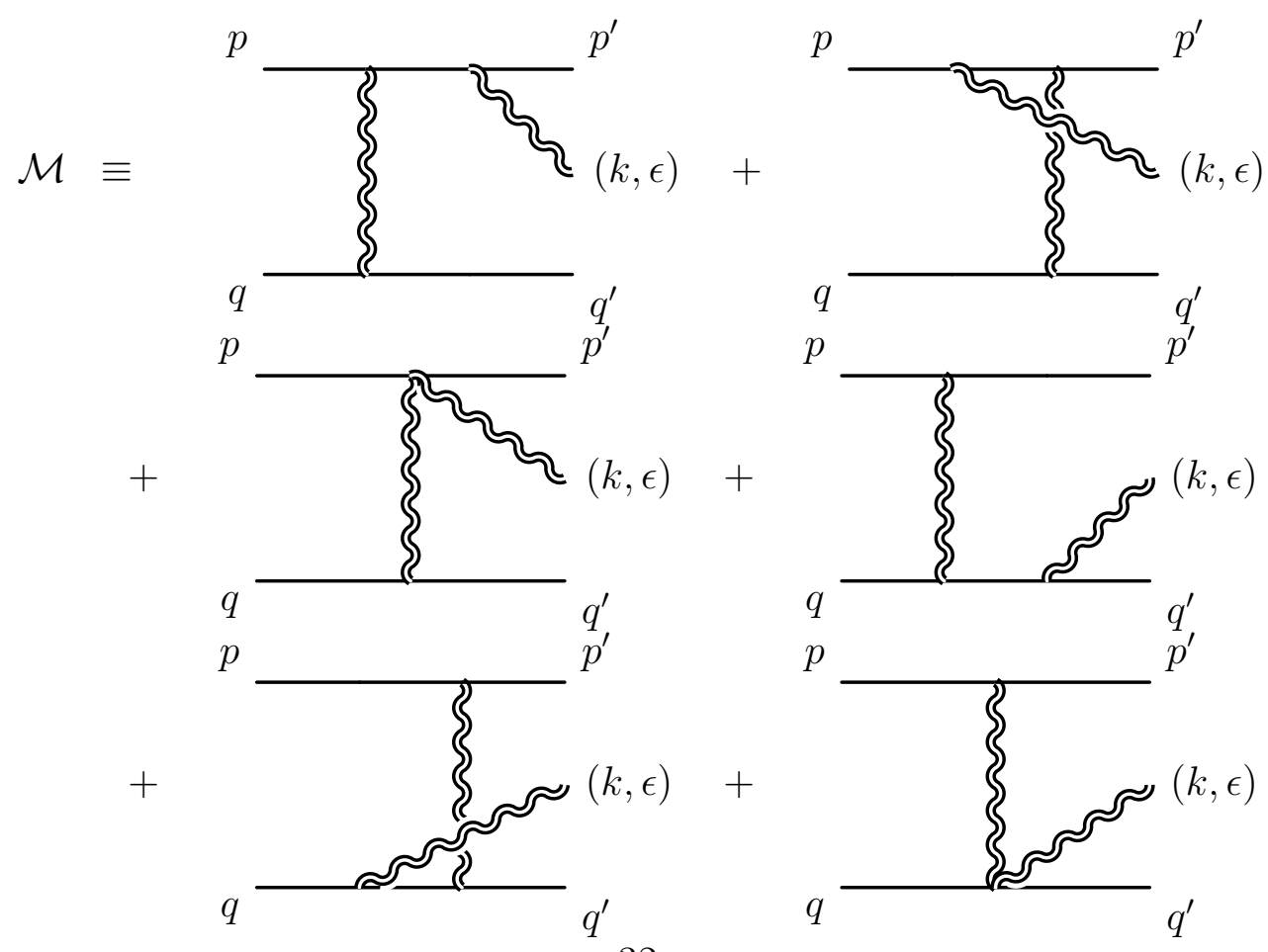




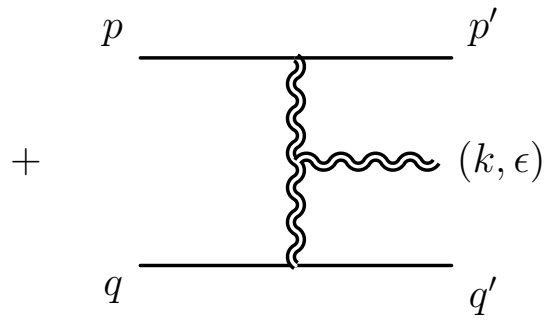

In the following we denote by $\mathcal{M}_{i}$ (with $i=1, \ldots, 7$ ) the contribution of each of these diagrams which we have calculated using the Feynman rules listed above. In order to recast the long expressions in a more convenient way, we introduce the momenta $k_{1}$ and $k_{2}$ defined in Eq. (2.39) and make use again of the Sudakov parametrization (2.40). Moreover, the graviton polarization tensor $\epsilon_{\mu \nu}(k)$ is written as

$$
\epsilon_{\mu \nu}(k)=\varepsilon_{\mu}(k) \varepsilon_{\nu}(k),
$$

where $\varepsilon(k) \cdot \varepsilon(k)=0$ and $k \cdot \varepsilon(k)=0$. Using the last condition to write

$$
\begin{aligned}
\varepsilon \cdot k_{1 \perp} & =\varepsilon \cdot k_{1 \perp}-\frac{1}{2} \varepsilon \cdot k \\
& =\frac{1}{2}\left[\varepsilon \cdot\left(k_{1 \perp}+k_{2 \perp}\right)-\left(\alpha_{1}-\alpha_{2}\right) \varepsilon \cdot p-\left(\beta_{1}-\beta_{2}\right) \varepsilon \cdot q\right], \\
\varepsilon \cdot k_{2 \perp} & =\varepsilon \cdot k_{2 \perp}+\frac{1}{2} \varepsilon \cdot k \\
& =\frac{1}{2}\left[\varepsilon \cdot\left(k_{1 \perp}+k_{2 \perp}\right)+\left(\alpha_{1}-\alpha_{2}\right) \varepsilon \cdot p+\left(\beta_{1}-\beta_{2}\right) \varepsilon \cdot q\right],
\end{aligned}
$$

the total amplitude $\mathcal{M}$ can be shown to have the structure

$$
\begin{aligned}
\mathcal{M} & =\left[\varepsilon \cdot\left(k_{1 \perp}+k_{2 \perp}\right)\right]\left[\varepsilon \cdot\left(k_{1 \perp}+k_{2 \perp}\right)\right] A_{k k}+\left[\varepsilon \cdot\left(k_{1 \perp}+k_{2 \perp}\right)\right](\varepsilon \cdot p) A_{k p} \\
& +\left[\varepsilon \cdot\left(k_{1 \perp}+k_{2 \perp}\right)\right](\varepsilon \cdot q) A_{k q}+(\varepsilon \cdot p)(\varepsilon \cdot p) A_{p p}+(\varepsilon \cdot q)(\varepsilon \cdot q) A_{q q} \\
& +(\varepsilon \cdot p)(\varepsilon \cdot q) A_{p q}
\end{aligned}
$$


The six coefficients $\mathcal{A}_{i i}$ appearing in this expression are rational functions of the momenta whose explicit form is given in Appendix A. The results are independent of the center of mass energy $s=(p+q)^{2}$ and can be written solely in terms of the Sudakov variables $\alpha_{1,2}, \beta_{1,2}$.

Before proceeding any further, we should point out that the computed amplitude is gauge invariant, i.e. using momentum conservation we have

$$
k_{\mu} \mathcal{M}^{\mu \nu}=0=k_{\nu} \mathcal{M}^{\mu \nu}
$$

where we have written $\mathcal{M}=\epsilon_{\mu \nu} \mathcal{M}^{\mu \nu}$. As a further cross-check of our calculations, we note that the total amplitude also satisfies the Steinmannn relations [57]. These are a consequence of unitarity and state that the amplitude cannot have simultaneous singularities, or multiple poles in energy variables, in overlapping channels. In our case the invariant masses associated with these two channels are

$$
s_{p^{\prime} k}=\left(p^{\prime}+k\right)^{2}, \quad s_{q^{\prime} k}=\left(q^{\prime}+k\right)^{2},
$$

which, in terms of the Sudakov variables, take the form

$$
s_{p^{\prime} k}=-s\left(\alpha_{2}+\beta_{2}\right), \quad s_{q^{\prime} k}=s\left(\alpha_{1}+\beta_{1}\right) .
$$

In a preliminary version of the expressions in Section A.1 it turned out that all six coefficients $\mathcal{A}_{i i}$ contained the potentially dangerous combination $\left(\alpha_{1}+\beta_{1}\right)\left(\alpha_{2}+\right.$ $\left.\beta_{2}\right)$ in their denominators. We explicitly checked, however, that the numerators vanish either when $\alpha_{1} \rightarrow-\beta_{1}$ or $\alpha_{2} \rightarrow-\beta_{2}$, canceling out one of the factors in the denominator and leaving behind simple poles in $s_{p^{\prime} k}$ or $s_{q^{\prime} k}$. We have finally simplified our expressions to explicitly show the non-existence of these unphysical poles. This fulfillment of Steinmann relations provides a highly nontrivial test of 
our results.

As in the QCD case analyzed above, our aim is to decompose the total amplitude in terms of gauge invariant combinations. Here we define

$$
\mathcal{M}_{\uparrow}=\mathcal{M}_{1}+\mathcal{M}_{2}+\mathcal{M}^{\prime} \quad \text { and } \quad \mathcal{M}_{\downarrow}=\mathcal{M}_{4}+\mathcal{M}_{5}+\mathcal{M}^{\prime \prime}
$$

where

$$
\mathcal{M}^{\prime}=\frac{t}{t-t^{\prime}}\left(\mathcal{M}_{3}+\mathcal{M}_{7}\right), \quad \mathcal{M}^{\prime \prime}=\frac{t^{\prime}}{t^{\prime}-t}\left(\mathcal{M}_{6}+\mathcal{M}_{7}\right)
$$

The total amplitude can be written as

$$
\mathcal{M}=\mathcal{M}_{\uparrow}+\mathcal{M}_{\downarrow}+\left(\frac{t^{\prime}}{t^{\prime}-t} \mathcal{M}_{3}+\frac{t}{t-t^{\prime}} \mathcal{M}_{6}\right)
$$

Remarkably, after a long calculation one can show that the last term cancels

$$
\frac{t^{\prime}}{t^{\prime}-t} \mathcal{M}_{3}+\frac{t}{t-t^{\prime}} \mathcal{M}_{6}=0
$$

To qualitatively understand this result we notice that both the scalar-scalargraviton and scalar-scalar-graviton-graviton vertices given earlier 2.2 are proportional to the same kinematical factor $p_{\mu} q_{\nu}+p_{\nu} q_{\mu}$. After working out all the index contractions in $\mathcal{M}_{3}$ and $\mathcal{M}_{6}$ it is possible to find that the only difference in the contributions of these two diagrams lies in the denominator of the interchanged graviton. Diagrammatically,

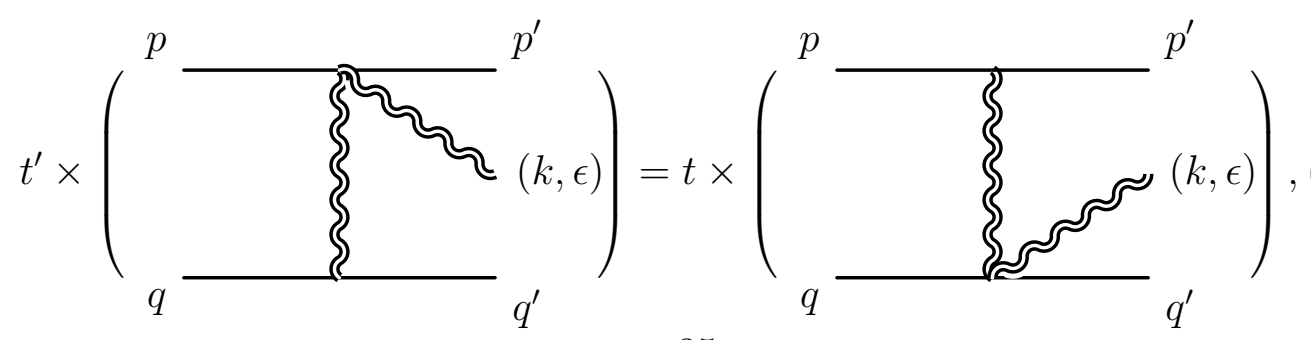


from where the cancellation in Eq. 2.62 follows.

We conclude that the amplitude can be written only in terms of the two contributions shown in Eq. 2.59)

$$
\mathcal{M}=\mathcal{M}_{\uparrow}+\mathcal{M}_{\downarrow}
$$

where each of the partial amplitudes $\mathcal{M}_{\uparrow}, \mathcal{M}_{\downarrow}$ have the structure shown in Eq. 2.55). Their corresponding coefficients are given in Appendix A.2. What makes this decomposition interesting is that both terms on the right-hand side are gauge invariant independently, i.e. they satisfy the Ward identities

$$
k_{\mu} \mathcal{M}_{\uparrow}^{\mu \nu}=0, \quad k_{\mu} \mathcal{M}_{\downarrow}^{\mu \nu}=0,
$$

where $\mathcal{M}_{\uparrow} \equiv \epsilon_{\mu \nu} \mathcal{M}_{\uparrow}^{\mu \nu}$ and $\mathcal{M}_{\downarrow} \equiv \epsilon_{\mu \nu} \mathcal{M}_{\downarrow}^{\mu \nu}$. As in the gauge theory case analyzed above, we can write the two gauge invariant contributions in terms of an effective vertex for the interaction of two on-shell scalars with one on-shell and one off-shell gravitons. The pictorial representation would be:
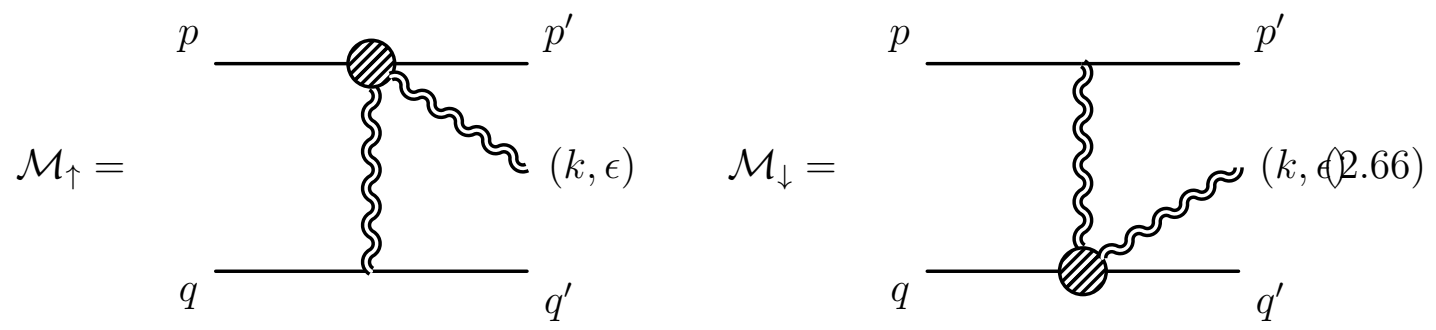

We find this result very interesting and are currently investigating the generalization of these effective diagrams to higher point amplitudes. If any iterative structure for higher order effective vertices could be found it would drastically 
help simplify loop calculations in Einstein-Hilbert gravity when using unitarity techniques.

\subsection{Gravity as a double copy of QCD in multi- Regge kinematics}

We are now ready to address a very interesting point which has attracted quite a lot of attention in recent literature: in which sense are our effective vertices in gravity and QCD related? It is not possible for us to answer this question directly in general kinematics since, in order to simplify our calculations, we have operated with very particular external states, scalars in Einstein-Hilbert and quarks in QCD. This issue has been discussed by Bern and collaborators in a series of works mainly involving the mapping of amplitudes with only gluons in one side and only gravitons in the other [52, 36, 58, 37]. We can, however, investigate our scattering amplitudes in multi-Regge kinematics and try to reproduce the results by Lipatov [23, 28, 29] where he found that the gluon and graviton emission vertices in MRK are indeed related as described in the following.

Let us make use of the relations $k_{1}^{2}=\beta_{1} s=t$ and $k_{2}^{2}=-\alpha_{2} s=t^{\prime}$ to rewrite the QCD MRK effective vertex of Eq. 2.49) in the form

$$
\Gamma_{\mu \sigma}^{\nu}=i g \eta_{\mu \sigma}\left[\left(\alpha_{1}-\frac{2 \beta_{1}}{\beta_{2}}\right) p^{\nu}+\left(\beta_{2}+\frac{2 \alpha_{2}}{\alpha_{1}}\right) q^{\nu}-\left(k_{1}+k_{2}\right)_{\perp}^{\nu}\right] \equiv i g \eta_{\mu \sigma} \Omega^{\nu}
$$

When looking for gravity as a simple double copy of the gauge theory amplitudes we find a difficulty in MRK. In this region, which corresponds to the limit of Sudakov parameters described in Eq. 2.41, we have that $s_{p^{\prime} k}=-\left(\alpha_{2}+\beta_{2}\right) s \simeq$ 
$-\beta_{2} s$ and $s_{q^{\prime} k}=\left(\alpha_{1}+\beta_{1}\right) s \simeq \alpha_{1} s$, and can write

$$
\Omega^{\nu} \simeq\left(\frac{s_{q^{\prime} k}}{s}+\frac{2 k_{1}^{2}}{s_{p^{\prime} k}}\right) p^{\nu}-\left(\frac{s_{p^{\prime} k}}{s}+\frac{2 k_{2}^{2}}{s_{q^{\prime} k}}\right) q^{\nu}-\left(k_{1}+k_{2}\right)_{\perp}^{\nu} .
$$

It is important to notice that, when written in this form, the naive double copy $\Omega^{\mu} \Omega^{\nu}$ has explicit unphysical double poles of the form $\left(s_{p^{\prime} k} s_{q^{\prime} k}\right)^{-1}$ which correspond to simultaneous energy discontinuities in overlapping channels. The solution proposed by Lipatov was to introduce a subtraction term in order to fulfill Steinmann relations which reads

$$
\mathcal{N}^{\mu}=2 \sqrt{k_{1}^{2} k_{2}^{2}}\left(\frac{p^{\mu}}{s_{p^{\prime} k}}-\frac{q^{\mu}}{s_{q^{\prime} k}}\right) \simeq-2 i \sqrt{\beta_{1} \alpha_{2}}\left(\frac{p^{\mu}}{\beta_{2}}+\frac{q^{\mu}}{\alpha_{1}}\right)
$$

with the corresponding double copy being

$$
\mathcal{N}^{\mu} \mathcal{N}^{\nu} \simeq-4 \beta_{1} \alpha_{2}\left(\frac{p^{\mu} p^{\nu}}{\beta_{2}^{2}}+\frac{q^{\mu} q^{\nu}}{\alpha_{1}^{2}}+\frac{p^{\mu} q^{\nu}+q^{\mu} p^{\nu}}{\alpha_{1} \beta_{2}}\right)
$$

What Lipatov showed is that the effective vertex for the emission of a graviton in Einstein-Hilbert gravity can be written in MRK as the following combination of QCD MRK effective vertices:

$$
\begin{aligned}
\Omega^{\mu} \Omega^{\nu}-\mathcal{N}^{\mu} \mathcal{N}^{\nu} & =\left(k_{1}+k_{2}\right)_{\perp}^{\mu}\left(k_{1}+k_{2}\right)_{\perp}^{\nu}+\left[\left(\alpha_{1}-\frac{2 \beta_{1}}{\beta_{2}}\right)^{2}+4 \frac{\beta_{1} \alpha_{2}}{\beta_{2}^{2}}\right] p^{\mu} p^{\nu} \\
& +\left[\left(\beta_{2}+\frac{2 \alpha_{2}}{\alpha_{1}}\right)^{2}+4 \frac{\beta_{1} \alpha_{2}}{\alpha_{1}^{2}}\right] q^{\mu} q^{\nu} \\
& +\left[\left(\alpha_{1}-\frac{2 \beta_{1}}{\beta_{2}}\right)\left(\beta_{2}+\frac{2 \alpha_{2}}{\alpha_{1}}\right)+4 \frac{\beta_{1} \alpha_{2}}{\alpha_{1} \beta_{2}}\right]\left(p^{\mu} q^{\nu}+q^{\mu} p^{\nu}\right) \\
& -\left(\alpha_{1}-\frac{2 \beta_{1}}{\beta_{2}}\right)\left(p^{\mu} k^{\nu}+k^{\mu} p^{\nu}\right)-\left(\beta_{2}+\frac{2 \alpha_{2}}{\alpha_{1}}\right)\left(q^{\mu} k^{\nu}+k^{\mu} q^{\nu}\right) .
\end{aligned}
$$

Let us see if this complicated structure is present in our exact calculations. For 
this purpose it is needed to find the corresponding coefficients in the expansion of our Eq. 2.55) which now we write in the form

$$
\begin{aligned}
\mathcal{M}^{\mu \nu} & =\left(k_{1}+k_{2}\right)_{\perp}^{\mu}\left(k_{1}+k_{2}\right)_{\perp}^{\nu}+\mathcal{A}_{p p} p^{\mu} p^{\nu}+\mathcal{A}_{q q} q^{\mu} q^{\nu}+\mathcal{A}_{p q}\left(p^{\mu} q^{\nu}+q^{\mu} p^{\nu}\right) \\
& +\mathcal{A}_{k p}\left(p^{\mu} k^{\nu}+k^{\mu} p^{\nu}\right)+\mathcal{A}_{k q}\left(q^{\mu} k^{\nu}+k^{\mu} q^{\nu}\right)
\end{aligned}
$$

where $\mathrm{E}^{1} \mathcal{A}_{i i}=A_{i i} / A_{k k}$.

Using the results for the exact gravitational amplitude given in the Appendix and operating in the MRK of Eq. (2.41), the Taylor series expansion of each of the coefficients in Eq. 2.72 is as follows:

$$
\begin{aligned}
\mathcal{A}_{p p} & =\alpha_{1}^{2}+\mathcal{O}\left(\alpha_{1}^{3}\right)+\beta_{1}\left[-\frac{4 \alpha_{1}}{\beta_{2}}+\frac{4 \alpha_{1}^{2}}{\beta_{2}}+\mathcal{O}\left(\alpha_{1}^{3}\right)\right] \\
& +\beta_{1}^{2}\left[\frac{4}{\beta_{2}^{2}}-\frac{12 \alpha_{1}}{\beta_{2}^{2}}+\frac{8 \alpha_{1}^{2}}{\beta_{2}^{2}}+\mathcal{O}\left(\alpha_{1}^{3}\right)\right]+\mathcal{O}\left(\beta_{1}^{3}\right) \\
& +\alpha_{2}\left\{-2 \alpha_{1}+\mathcal{O}\left(\alpha_{1}^{3}\right)+\left[\frac{8 \beta_{2}+4}{\beta_{2}^{2}}-\frac{\left(8 \beta_{2}+4\right) \alpha_{1}}{\beta_{2}^{2}}+\mathcal{O}\left(\alpha_{1}^{3}\right)\right] \beta_{1}\right. \\
& \left.+\left[\frac{4\left(\beta_{2}+1\right)}{\beta_{2}^{2} \alpha_{1}}+\frac{4-8 \beta_{2}^{2}}{\beta_{2}^{3}}+\frac{4 \alpha_{1}}{\beta_{2}}-\frac{4\left(\beta_{2}+1\right) \alpha_{1}^{2}}{\beta_{2}^{3}}+\mathcal{O}\left(\alpha_{1}^{3}\right)\right] \beta_{1}^{2}+\mathcal{O}\left(\beta_{1}^{3}\right)\right\} \\
& +\mathcal{O}\left(\alpha_{2}^{2}\right) \simeq \alpha_{1}^{2}-\frac{4 \alpha_{1} \beta_{1}}{\beta_{2}}+\frac{4 \beta_{1}^{2}}{\beta_{2}^{2}}+\frac{4 \alpha_{2} \beta_{1}}{\beta_{2}^{2}}+\ldots \\
\mathcal{A}_{q q} & =\beta_{2}^{2}+\mathcal{O}\left(\beta_{2}^{3}\right)+\beta_{1}\left[-2 \beta_{2}+\mathcal{O}\left(\beta_{2}^{3}\right)\right]+\mathcal{O}\left(\beta_{1}^{2}\right) \\
& +\alpha_{2}\left\{\frac{4 \beta_{2}}{\alpha_{1}}+\frac{4 \beta_{2}^{2}}{\alpha_{1}}+\mathcal{O}\left(\beta_{2}^{3}\right)+\left[\frac{4-8 \alpha_{1}}{\alpha_{1}^{2}}+\frac{\left(4-8 \alpha_{1}\right) \beta_{2}}{\alpha_{1}^{2}}+\mathcal{O}\left(\beta_{2}^{3}\right)\right] \beta_{1}\right. \\
& \left.+\mathcal{O}\left(\beta_{1}^{2}\right)\right\}+\alpha_{2}^{2}\left\{\frac{4}{\alpha_{1}^{2}}+\frac{12 \beta_{2}}{\alpha_{1}^{2}}+\frac{8 \beta_{2}^{2}}{\alpha_{1}^{2}}+\mathcal{O}\left(\beta_{2}^{3}\right)\right.
\end{aligned}
$$

\footnotetext{
${ }^{1}$ The process dependent impact factors can be evaluated from the MRK limit of the normalization factor $A_{k k}$.
} 


$$
\begin{aligned}
& \left.+\left[\frac{4-4 \alpha_{1}}{\alpha_{1}^{2} \beta_{2}}+\frac{4-8 \alpha_{1}^{2}}{\alpha_{1}^{3}}-\frac{4 \beta_{2}}{\alpha_{1}}+\frac{4\left(\alpha_{1}-1\right) \beta_{2}^{2}}{\alpha_{1}^{3}}+\mathcal{O}\left(\beta_{2}^{3}\right)\right] \beta_{1}+\mathcal{O}\left(\beta_{1}^{2}\right)\right\} \\
& +\mathcal{O}\left(\alpha_{2}^{3}\right) \simeq \beta_{2}^{2}+\frac{4 \alpha_{2} \beta_{2}}{\alpha_{1}}+\frac{4 \alpha_{2} \beta_{1}}{\alpha_{1}^{2}}+\frac{4 \alpha_{2}^{2}}{\alpha_{1}^{2}}+\ldots
\end{aligned}
$$

$$
\begin{aligned}
\mathcal{A}_{p q} & =\alpha_{1} \beta_{2}+\mathcal{O}\left(\beta_{2}^{2}\right)+\left(\alpha_{1}-2\right) \beta_{1}+\mathcal{O}\left(\beta_{1}^{2}\right) \\
& +\alpha_{2}\left[2+\beta_{2}+\mathcal{O}\left(\beta_{2}^{2}\right)+\beta_{1}+\mathcal{O}\left(\beta_{1}^{2}\right)\right]+\mathcal{O}\left(\alpha_{2}^{2}\right) \\
& \simeq \alpha_{1} \beta_{2}-2 \beta_{1}+2 \alpha_{2}+\ldots \\
\mathcal{A}_{k p} & =-\alpha_{1}+\beta_{1}\left[\frac{2-2 \alpha_{1}}{\beta_{2}}+\mathcal{O}\left(\beta_{2}^{2}\right)+\mathcal{O}\left(\beta_{1}^{2}\right)\right]+\mathcal{O}\left(\alpha_{2}^{1}\right) \\
& \simeq-\alpha_{1}+\frac{2 \beta_{1}}{\beta_{2}}+\ldots \\
\mathcal{A}_{k q} & =-\beta_{2}+\mathcal{O}\left(\beta_{2}^{2}\right)+\mathcal{O}\left(\beta_{1}^{1}\right)+\alpha_{2}\left[-\frac{2}{\alpha_{1}}-\frac{2 \beta_{2}}{\alpha_{1}}+\mathcal{O}\left(\beta_{2}^{2}\right)+\mathcal{O}\left(\beta_{1}^{1}\right)\right]+\mathcal{O}\left(\alpha_{2}^{2}\right) \\
& \simeq-\beta_{2}-\frac{2 \alpha_{2}}{\alpha_{1}}+\ldots
\end{aligned}
$$

These coefficients calculated in MRK are therefore in exact agreement with those of Eq. 2.71). This is a highly non-trivial check of our calculation which sheds light on the deep relation between Einstein-Hilbert gravity and gauge theories.

The procedure we have followed in this work to cross-check our calculations can be applied to amplitudes with an arbitrary number of loops and external legs. We have found that the representation of the exact amplitudes in Sudakov variables facilitates the application of the MRK limit and the comparison with the iterated form of amplitudes valid in this region. This adds to the more standard checks related to gauge invariance and agreement with the Steinmann relations. 
In this chapter we have evaluated the tree level five-point amplitude for the scattering of two distinct scalars with a graviton in the final state considering Einstein-Hilbert gravity as a usual gauge theory. The calculation has been performed in exact kinematics with the final result expressed in Sudakov variables. We have tested not only the gauge invariance of the full amplitude but also the lack of simultaneous singularities in overlapping channels, in agreement with Steinmann relations.

We have found the remarkable result that, due to the subtle cancellation shown in Eq. 2.62, the full amplitude can be written as the sum of only the two gauge invariant topologies given in Eq. (2.66), both written in terms of the same effective vertex for graviton emission off a scalar line together with an off-shell graviton connecting with the other distinct scalar line. A natural expansion for a general five-point amplitude in gravity is given in Eq. 2.55) with the coefficients for our full result being shown in Section A.1. The coefficients for the separation into our two novel effective topologies are explicitly written in Section A.2. This new structure in terms of effective vertices will be useful to simplify and streamline the calculation of higher order corrections in gravity, reducing in a great amount the number of possible topologies contributing to a given process. It is likely that new effective topologies will appear as the number of external legs in the amplitude increases. The possible relations of the new effective vertices with the lower order ones will be the subject of our future investigations.

In order to investigate the interesting link between gravity and gauge theories we have also offered a detailed derivation of the QCD amplitude with four external quarks and one gluon. In this case a similar separation into effective topologies as in Einstein-Hilbert gravity appears. We have reproduced the results obtained by Lipatov many years ago [23, 25, 28, 29] by showing that the graviton emission vertex in multi-Regge kinematics can be written as the product of two gluon 
emission vertices in QCD also in the same limit, with an additional subtraction needed to fulfill the Steinmann relations. 


\section{Chapter 3}

\section{Color-Kinematics Duality and Scalar QCD}

\subsection{Extending the BCJ prescription}

As explained in Chapter 1, a surprising relation between gluon and graviton amplitudes, dubbed color-kinematics duality, has been found by Bern, Carrasco, and Johansson (BCJ) [35] (see also [36, 38, 37, 39, 40]). Here we remind the reader of the main features of this duality, introducing the notation to be used in this chapter.

The tree-level $n$-gluon amplitudes have the general structure

$$
\mathcal{A}(1, \ldots, n)_{\text {tree }}=g^{n-2} \sum_{i \in \Gamma} \frac{C_{i} N_{i}}{\prod_{\alpha} s_{\alpha}},
$$

where the sum runs over all Feynman diagram topologies $\Gamma, C_{i}$ are their color factors, $N_{i}$ the corresponding numerators, and $s_{\alpha}$ the kinematic invariants associated to the internal propagators of each diagram. Choosing the phases of the 
color factors properly, they satisfy Jacobi identities of the form

$$
C_{i}+C_{j}+C_{k}=0
$$

for certain triplets of indices $(i, j, k)$.

The amplitude (3.1) is invariant under generalized gauge transformations [35, 38. shifting the numerators in the form $N_{i} \rightarrow N_{i}+\Delta_{i}$, where $\Delta_{i}$ are functions of the momenta satisfying

$$
\sum_{i \in T} \frac{C_{i} \Delta_{i}}{\prod_{\alpha} s_{\alpha}}=0 .
$$

These are generalizations of the standard gauge transformations shifting the gluon polarization vectors $\varepsilon_{\mu}(k) \rightarrow \varepsilon_{\mu}(k)+\lambda k_{\mu}$, with $\lambda$ being a function of the momenta. This new freedom can be used to choose the numerators $N_{i}$ such that they replicate the Jacobi identities of the corresponding color factors shown in Eq. (3.2), namely

$$
N_{i}+N_{j}+N_{k}=0
$$

for the same triplets $(i, j, k)$. The numerators obtained by the direct application of the Feynman rules in QCD do not fulfill these BCJ duality relations. In Ref. [38] a nonlocal action for the gluon field was constructed whose Feynman rules give numerators satisfying (3.4) for the five gluon tree-level amplitude.

Color-kinematics duality provides a prescription to construct the amplitude for the tree-level scattering of $n$ gravitons from two copies of the tree-level amp- 
litude of $n$ gluons as [35]

$$
-i \mathcal{M}(1, \ldots, n)_{\text {tree }}=\left(\frac{\kappa}{2}\right)^{n-2} \sum_{i \in \Gamma} \frac{N_{i} \widetilde{N}_{i}}{\prod_{\alpha} s_{\alpha}}
$$

Here $N_{i}, \widetilde{N}_{i}$ are two replicas of the numerators of the gauge theory amplitude (3.1), one of them at least, satisfying the BCJ duality relations (3.4), and $\kappa$ the gravitational coupling. This hidden connection between gauge theories and gravity amplitudes is very remarkable since, unlike the KLT relations, it is expected to hold also for loop amplitudes before integration over the loop momenta.

Whenever possible it is important to test the validity of this intriguing duality. Different kinematic limits of scattering amplitudes either in gauge or gravitational amplitudes are known which can serve as a test ground for the BCJ procedure. One recent example is the soft limit investigated in [59]. In Chapter 3 we focused on the multi-Regge limit, which is well understood both in the gauge [20, 21, 22] and gravitational sides [25, 23, 28, 29, 60, 24].

At loop level, $\mathcal{N}$-supergravity amplitudes have been constructed from those of Yang-Mills theories even in the case of non-maximally supersymmetric theories [61]. In [60] it has been shown that the four-graviton amplitudes at two loops calculated using the BCJ duality for $\mathcal{N} \geq 4$ generate the correct Regge limit even at double logarithmic (in energy) accuracy, which goes beyond the well-known exponentiation of infrared divergences. In the same work predictions have been made for graviton scattering in all supergravities and Einstein-Hilbert gravity to arbitrary order in the gravitational coupling which should serve as a good test of the color-kinematics duality at higher orders.

In Chapter 2 of this thesis we have carried out a study of exact inelastic amplitudes both in QCD and Einstein-Hilbert gravity [24]. Using a representation in Sudakov variables, we were able to reproduce the results of Lipatov for the 
emission of a graviton in MRK. It turns out that this limit can be related to that of gluon production in QCD and an interesting double-copy structure emerges. In what follows we investigate the color-kinematics duality in SQCD by studying the scattering of two distinct scalars with the associated emission of a gluon in MRK, in this way it is possible to understand which pieces are somehow universal in the Regge limit and which ones are dependent on the matter content of the gauge theory when applying the BCJ procedure [62].

\section{2 sQCD and color-kinematics duality}

Our starting point is the tree-level contribution to the scattering of two distinguishable massless scalar particles with the emission of one gluon in sQCD

$$
\Phi(p, j)+\Phi^{\prime}(q, m) \longrightarrow \Phi\left(p^{\prime}, i\right)+\Phi\left(q^{\prime}, i\right)+g(k, a, \varepsilon)
$$

where in brackets we indicated the momenta and color quantum numbers of the involved particles, as well as the polarization vector in the case of the gluon. We choose this amplitude in order to reduce the number of diagrams to be calculated and to understand how the BCJ procedure fails when in the external states we not only have gluons but also scalar fields. The amplitude receives contributions from the following seven diagrams

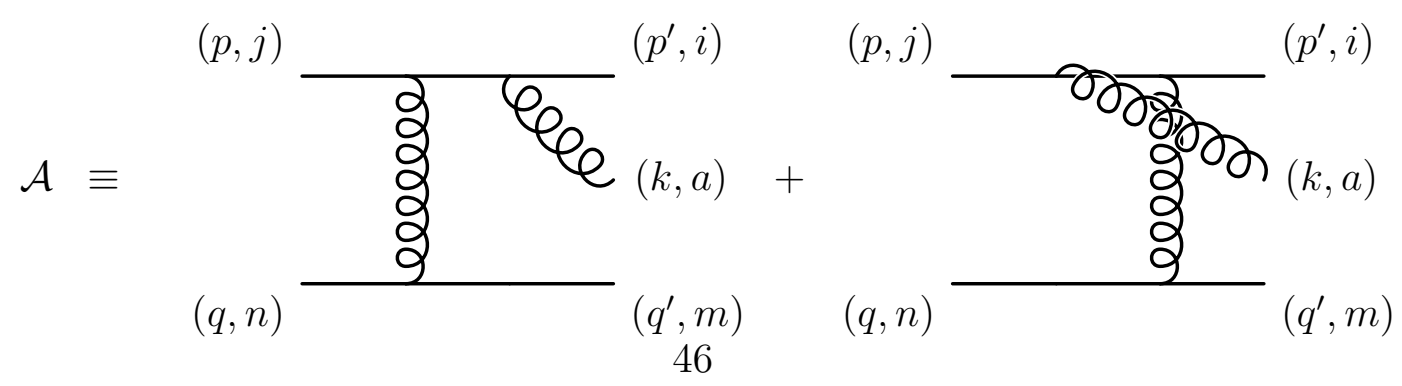



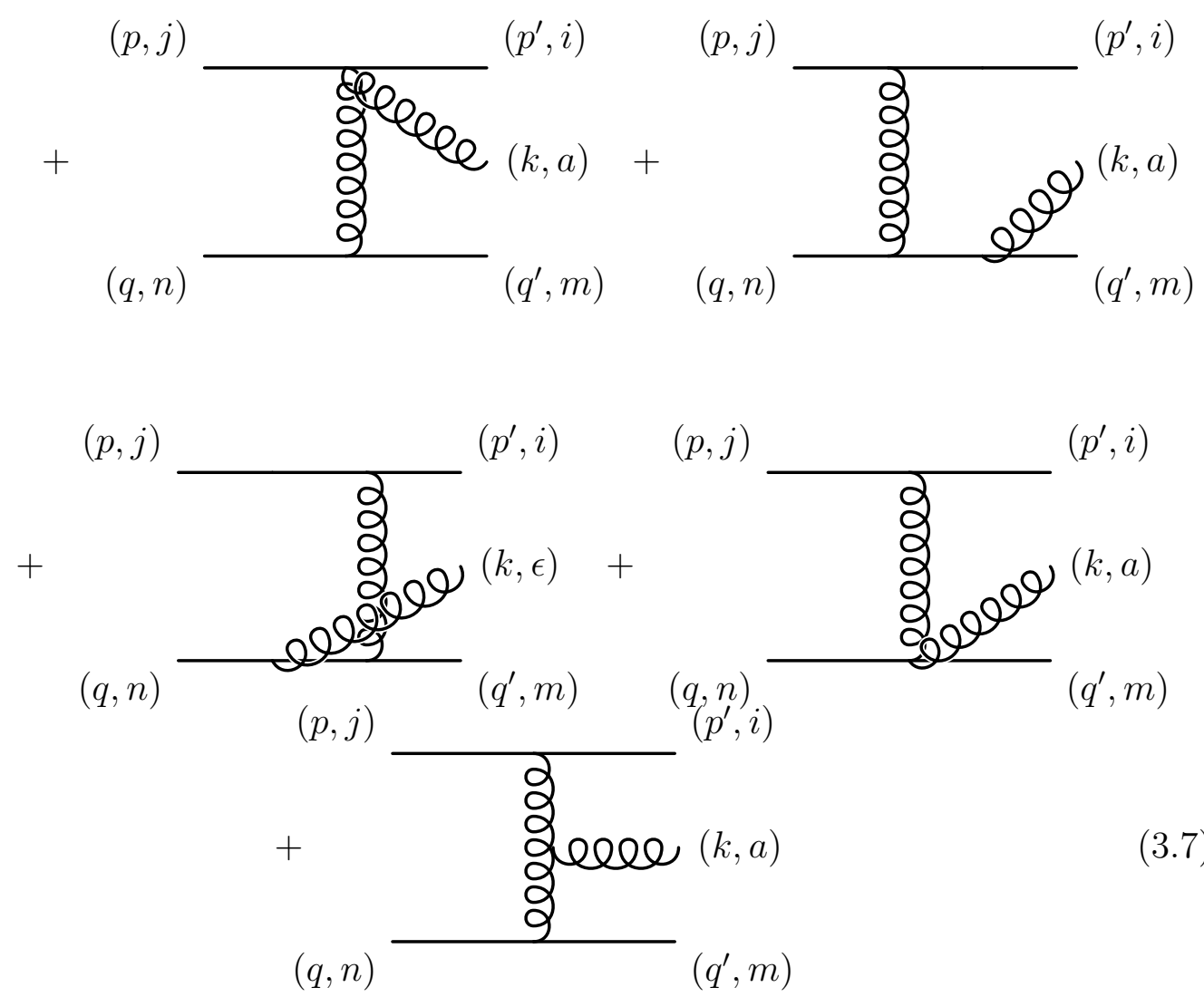

and has the general structure

$$
\mathcal{A}=g^{3}\left(\frac{C_{1} N_{1}}{t^{\prime} s_{p^{\prime} k}}+\frac{C_{2} N_{2}}{t^{\prime} s_{p k}}+\frac{C_{3} N_{3}}{t^{\prime}}+\frac{C_{4} N_{4}}{t s_{q^{\prime} k}}+\frac{C_{5} N_{5}}{t s_{q k}}+\frac{C_{6} N_{6}}{t}+\frac{C_{7} N_{7}}{t t^{\prime}}\right)
$$

where the numbering corresponds to the order in which the diagrams appear in Eq. (3.7). In writing the amplitude we have introduced the following kinematic invariants

$$
\begin{aligned}
t & =\left(p-p^{\prime}\right)^{2}, \\
t^{\prime} & =\left(q-q^{\prime}\right)^{2}, \\
s_{p k} & =(p+k)^{2}, \\
s_{p^{\prime} k} & =\left(p^{\prime}+k\right)^{2}, \\
s_{q k} & =(q+k)^{2},
\end{aligned}
$$




$$
s_{q^{\prime} k}=\left(q^{\prime}+k\right)^{2},
$$

and color factors

$$
\begin{aligned}
& C_{1}=T_{i k}^{a} T_{k j}^{b} T_{m n}^{b}, \\
& C_{2}=T_{i k}^{b} T_{k j}^{a} T_{m n}^{b}, \\
& C_{3}=T_{i k}^{a} T_{k j}^{b} T_{m n}^{b}+T_{i k}^{b} T_{k j}^{a} T_{m n}^{b}, \\
& C_{4}=T_{i j}^{b} T_{m k}^{a} T_{k n}^{b}, \\
& C_{5}=T_{i j}^{b} T_{m k}^{b} T_{k n}^{a}, \\
& C_{6}=T_{i j}^{b} T_{m k}^{a} T_{k n}^{b}+T_{i j}^{b} T_{m k}^{b} T_{k n}^{a}, \\
& C_{7}=i f^{a b c} T_{i j}^{b} T_{m n}^{c},
\end{aligned}
$$

with $T_{i j}^{a}$ being the generators of the representation in which the scalar fields transform. They satisfy the Jacobi-like identities

$$
\begin{aligned}
& C_{1}-C_{2}+C_{7}=0, \\
& C_{1}+C_{2}-C_{3}=0, \\
& C_{4}-C_{5}-C_{7}=0, \\
& C_{4}+C_{5}-C_{6}=0 .
\end{aligned}
$$

Applying the Feynman rules ${ }^{1}$ of sQCD, we find the following form for the numerators $N_{i}$

$$
\begin{aligned}
& N_{1}=2 i\left[\left(p+p^{\prime}+k\right) \cdot\left(q+q^{\prime}\right)\right]\left[p^{\prime} \cdot \varepsilon(k)\right], \\
& N_{2}=-2 i\left[\left(p+p^{\prime}-k\right) \cdot\left(q+q^{\prime}\right)\right][p \cdot \varepsilon(k)],
\end{aligned}
$$

\footnotetext{
${ }^{1}$ See, e.g., 63. Notice, however, that in the conventions of this reference the gauge group generators are anti-Hermitian, whereas we take them Hermitian.
} 


$$
\begin{aligned}
N_{3} & =-i\left(q+q^{\prime}\right) \cdot \varepsilon(k), \\
N_{4} & =2 i\left[\left(q+q^{\prime}+k\right) \cdot\left(p+p^{\prime}\right)\right]\left[q^{\prime} \cdot \varepsilon(k)\right], \\
N_{5} & =-2 i\left[\left(q+q^{\prime}-k\right) \cdot\left(p+p^{\prime}\right)\right][q \cdot \varepsilon(k)], \\
N_{6} & =-i\left(p+p^{\prime}\right) \cdot \varepsilon(k), \\
N_{7} & =-i\left\{\left[\left(q+q^{\prime}\right) \cdot\left(p-p^{\prime}+k\right)\right]\left[\left(p+p^{\prime}\right) \cdot \varepsilon(k)\right]\right. \\
& +\left[\left(p+p^{\prime}\right) \cdot\left(q+q^{\prime}\right)\right]\left[\left(p^{\prime}-p-q^{\prime}+q\right) \cdot \varepsilon(k)\right] \\
& \left.+\left[\left(p+p^{\prime}\right) \cdot\left(q^{\prime}-q-k\right)\right]\left[\left(q+q^{\prime}\right) \cdot \varepsilon(k)\right]\right\} .
\end{aligned}
$$

These numerators do not satisfy the BCJ duality relations derived from Eq. (3.11) and are therefore not ready to apply the color-kinematic duality prescription. The generalized nonlocal gauge transformation

$$
\begin{aligned}
& N_{1}^{\prime}=N_{1}+s_{p^{\prime} k}\left(N_{3}-\frac{N_{7}}{2 t}\right), \\
& N_{2}^{\prime}=N_{2}+s_{p k}\left(N_{3}+\frac{N_{7}}{2 t}\right), \\
& N_{3}^{\prime}=N_{3}, \\
& N_{4}^{\prime}=N_{4}+s_{q^{\prime} k}\left(N_{6}+\frac{N_{7}}{2 t^{\prime}}\right), \\
& N_{5}^{\prime}=N_{5}+s_{q k}\left(N_{6}-\frac{N_{7}}{2 t^{\prime}}\right), \\
& N_{6}^{\prime}=N_{6}, \\
& N_{7}^{\prime}=N_{7},
\end{aligned}
$$

recasts the amplitude in terms of only four color factors

$$
\mathcal{A}=g^{3}\left(\frac{C_{1} N_{1}^{\prime}}{t^{\prime} s_{p^{\prime} k}}+\frac{C_{2} N_{2}^{\prime}}{t^{\prime} s_{p k}}+\frac{C_{4} N_{4}^{\prime}}{t s_{q^{\prime} k}}+\frac{C_{5} N_{5}^{\prime}}{t s_{q k}}\right),
$$


satisfying the single Jacobi identity (derived from Eq. (3.11)

$$
C_{1}-C_{2}+C_{4}-C_{5}=0
$$

These new numerators $N_{i}^{\prime}$ do not comply with BCJ duality. To fix this we perform a further generalized gauge transformation of the form

$$
\begin{aligned}
& N_{1}^{\prime \prime}=N_{1}^{\prime}+\alpha t^{\prime} s_{p^{\prime} k}, \\
& N_{2}^{\prime \prime}=N_{2}^{\prime}-\alpha t^{\prime} s_{p k}, \\
& N_{4}^{\prime \prime}=N_{4}+\alpha t s_{q^{\prime} k}, \\
& N_{5}^{\prime \prime}=N_{5}-\alpha t s_{q k},
\end{aligned}
$$

where the function $\alpha$ is determined by requiring

$$
N_{1}^{\prime \prime}-N_{2}^{\prime \prime}+N_{3}^{\prime \prime}-N_{4}^{\prime \prime}=0
$$

This gives

$$
\alpha=\frac{-N_{1}^{\prime}+N_{2}^{\prime}-N_{4}^{\prime}+N_{5}^{\prime}}{t^{\prime}\left(s_{p k}+s_{p^{\prime} k}\right)+t\left(s_{q k}+s_{q^{\prime} k}\right)} .
$$

After all these algebraic manipulations we have managed to write our amplitude in the form

$$
\mathcal{A}=g^{3}\left(\frac{C_{1} N_{1}^{\prime \prime}}{t^{\prime} s_{p^{\prime} k}}+\frac{C_{2} N_{2}^{\prime \prime}}{t^{\prime} s_{p k}}+\frac{C_{4} N_{4}^{\prime \prime}}{t s_{q^{\prime} k}}+\frac{C_{5} N_{5}^{\prime \prime}}{t s_{q k}}\right)
$$

where the numerators satisfy (3.17). The next step is to apply the color-kinematics 
duality prescription to construct the gravitational amplitude, i.e.

$$
-i \mathcal{M}=\left(\frac{\kappa}{2}\right)^{3}\left(\frac{N_{1}^{\prime \prime} \widetilde{N}_{1}^{\prime \prime}}{t^{\prime} s_{p^{\prime} k}}+\frac{N_{2}^{\prime \prime} \widetilde{N_{2}^{\prime \prime}}}{t^{\prime} s_{p k}}+\frac{N_{4}^{\prime \prime} \widetilde{N}_{4}^{\prime \prime}}{t s_{q^{\prime} k}}+\frac{N_{5}^{\prime \prime} \widetilde{N_{5}^{\prime \prime}}}{t s_{q k}}\right) .
$$

The graviton polarization tensor $\epsilon_{\mu \nu}(k)$ is reconstructed as

$$
\varepsilon_{\mu}(k) \widetilde{\varepsilon}_{\nu}(k) \longrightarrow \epsilon_{\mu \nu}(k),
$$

with $\varepsilon_{\mu}(k), \widetilde{\varepsilon}_{\mu}(k)$ being the gluon polarization vectors contained in the numerators $N_{i}^{\prime \prime}$ and $\widetilde{N_{i}^{\prime \prime}}$.

At this point it is convenient to redefine the momenta according to

$$
p^{\prime}=p-k_{1}, \quad q^{\prime}=q+k_{2}, \quad k=k_{1}-k_{2}
$$

and write $k_{1}$ and $k_{2}$ using Sudakov parameters

$$
k_{1}^{\mu}=\alpha_{1} p^{\mu}+\beta_{1} q^{\mu}+k_{1, \perp}^{\mu}, \quad k_{2}^{\mu}=\alpha_{2} p^{\mu}+\beta_{2} q^{\mu}+k_{2, \perp}^{\mu} .
$$

In this representation for the momenta, the amplitude

$$
-i \mathcal{M} \equiv-i A_{k k} \mathcal{M}^{\mu \nu} \epsilon_{\mu \nu}(k)
$$

can be shown to have the following tensor structure

$$
\begin{aligned}
\mathcal{M}^{\mu \nu} & =\left(k_{1}+k_{2}\right)_{\perp}^{\mu}\left(k_{1}+k_{2}\right)_{\perp}^{\nu}+\mathcal{A}_{k p}\left[\left(k_{1}+k_{2}\right)_{\perp}^{\mu} p^{\nu}+p^{\mu}\left(k_{1}+k_{2}\right)_{\perp}^{\nu}\right] \\
& +\mathcal{A}_{k q}\left[\left(k_{1}+k_{2}\right)_{\perp}^{\mu} q^{\nu}+q^{\mu}\left(k_{1}+k_{2}\right)_{\perp}^{\nu}\right]+\mathcal{A}_{p q}\left(p^{\mu} q^{\nu}+q^{\mu} p^{\nu}\right) \\
& +\mathcal{A}_{q q} q^{\mu} q^{\nu}+\mathcal{A}_{p p} p^{\mu} p^{\nu} .
\end{aligned}
$$


Let us point out that in Eq. (3.24) we have factored out the coefficient $A_{k k}$ of the term proportional to $\left(k_{1}+k_{2}\right)_{\perp}^{\mu}\left(k_{1}+k_{2}\right)_{\perp}^{\nu}$. In the MRK limit

$$
1 \gg \alpha_{1} \gg \alpha_{2}, \quad 1 \gg\left|\beta_{2}\right| \gg\left|\beta_{1}\right|
$$

the prefactors $\mathcal{A}_{i}$ have the following form

$$
\begin{aligned}
& \mathcal{A}_{p p}=\left(\alpha_{1}-2 \frac{\beta_{1}}{\beta_{2}}\right)^{2}+2 \alpha_{2} \beta_{1}\left(\frac{\alpha_{1}+\beta_{2}}{\beta_{2}^{2}}\right)+\ldots, \\
& \mathcal{A}_{q q}=\left(\beta_{2}+2 \frac{\alpha_{2}}{\alpha_{1}}\right)^{2}-2 \alpha_{2} \beta_{1}\left(\frac{\alpha_{1}+\beta_{2}}{\alpha_{1}^{2}}\right)+\ldots, \\
& \mathcal{A}_{p q}=\left(\alpha_{1}-2 \frac{\beta_{1}}{\beta_{2}}\right)\left(\beta_{2}+2 \frac{\alpha_{2}}{\alpha_{1}}\right)+\ldots, \\
& \mathcal{A}_{k p}=-\left(\alpha_{1}-2 \frac{\beta_{1}}{\beta_{2}}\right)+\ldots \\
& \mathcal{A}_{k q}=-\left(\beta_{2}+2 \frac{\alpha_{2}}{\alpha_{1}}\right)+\ldots
\end{aligned}
$$

We want to compare these results, obtained after applying the BCJ colorkinematics duality, with the ones found in Section 2.5 using traditional Feynman rules for the gravitational scattering of two different scalars with emission of a graviton [24]:

$$
\Phi(p)+\Phi^{\prime}(q) \longrightarrow \Phi\left(p^{\prime}\right)+\Phi^{\prime}\left(q^{\prime}\right)+G(k, \epsilon)
$$

There we obtained that the tree-level amplitude in the MRK regime can be written as (in the notation of Eq. (3.24))

$$
\mathcal{M}^{\mu \nu}=\Omega^{\mu} \Omega^{\nu}-\mathcal{N}^{\mu} \mathcal{N}^{\nu},
$$


where

$$
\Omega^{\mu}=\left(\alpha_{1}-\frac{2 \beta_{1}}{\beta_{2}}\right) p^{\mu}+\left(\beta_{2}+\frac{2 \alpha_{2}}{\alpha_{1}}\right) q^{\mu}-\left(k_{1}+k_{2}\right)_{\perp}^{\mu}
$$

is Lipatov's MRK effective vertex for the coupling of two Reggeized gluons to an on-shell gluon in QCD, and

$$
\mathcal{N}^{\mu}=-2 i \sqrt{\beta_{1} \alpha_{2}}\left(\frac{p^{\mu}}{\beta_{2}}+\frac{q^{\mu}}{\alpha_{1}}\right)
$$

As explained, the term $\mathcal{N}^{\mu} \mathcal{N}^{\nu}$ in Eq. 1.20 is responsible for the cancellation of overlapping singularities in simultaneous channels required by the Steinmann relations [57] (see also [64, 65] for a recent discussion). It is important to realize that Eq. (3.27) does not reproduce the full structure indicated in Eq. (1.20). This is an effect of applying the color-kinematics duality to an amplitude where some of the external states are not gluons in the gauge theory side. However, we can see that the term corresponding purely to the "square" of the gauge vertex, $\Omega^{\mu} \Omega^{\nu}$, is correctly reproduced by the BCJ prescription in the Regge limit.

In order to show that, although the $\mathcal{N}^{\mu} \mathcal{N}^{\nu}$ terms are not given by BCJ colorkinematic duality, the $\Omega^{\mu} \Omega^{\nu}$ contributions are indeed retrieved, we apply the prescription to a set of numerators satisfying different BCJ duality identities. Using the local generalized gauge transformation

$$
\begin{aligned}
& N_{1}^{\prime}=N_{1}+s_{p^{\prime} k} N_{3}, \\
& N_{2}^{\prime}=N_{2}+s_{p k} N_{3}, \\
& N_{3}^{\prime}=N_{3}, \\
& N_{4}^{\prime}=N_{4}+s_{q^{\prime} k} N_{6}, \\
& N_{5}^{\prime}=N_{5}+s_{q k} N_{6},
\end{aligned}
$$




$$
N_{7}^{\prime}=N_{7}
$$

the sQCD amplitude can be written in the form

$$
\mathcal{A}=g^{3}\left(\frac{C_{1} N_{1}^{\prime}}{t^{\prime} s_{p^{\prime} k}}+\frac{C_{2} N_{2}^{\prime}}{t^{\prime} s_{p k}}+\frac{C_{4} N_{4}^{\prime}}{t s_{q^{\prime} k}}+\frac{C_{5} N_{5}^{\prime}}{t s_{q k}}+\frac{C_{7} N_{7}^{\prime}}{t t^{\prime}}\right)
$$

A further transformation

$$
\begin{aligned}
& N_{1}^{\prime \prime}=N_{1}^{\prime}+\alpha t^{\prime} s_{p^{\prime} k}, \\
& N_{2}^{\prime \prime}=N_{2}^{\prime}-\alpha t^{\prime} s_{p k}, \\
& N_{4}^{\prime \prime}=N_{4}^{\prime}-\beta t s_{q^{\prime} k}, \\
& N_{5}^{\prime \prime}=N_{5}^{\prime}+\beta t s_{q k}, \\
& N_{7}^{\prime \prime}=N_{7}^{\prime}+(\alpha+\beta) t t^{\prime},
\end{aligned}
$$

is performed, where $\alpha$ and $\beta$ are rational functions of the kinematic invariants fully determined by the condition that the numerators $N_{i}^{\prime \prime}$ satisfy BCJ duality

$$
N_{1}^{\prime \prime}-N_{2}^{\prime \prime}+N_{7}^{\prime \prime}=0, \quad N_{4}^{\prime \prime}-N_{5}^{\prime \prime}-N_{7}^{\prime \prime}=0 .
$$

We apply now color-kinematics duality to construct the amplitude:

$$
-i \mathcal{M}=\left(\frac{\kappa}{2}\right)^{3}\left(\frac{N_{1}^{\prime \prime} \widetilde{N}_{1}^{\prime \prime}}{t^{\prime} s_{p^{\prime} k}}+\frac{N_{2}^{\prime \prime} \widetilde{N}_{2}^{\prime \prime}}{t^{\prime} s_{p k}}+\frac{N_{4}^{\prime \prime} \widetilde{N_{2}^{\prime \prime}}}{t s_{q^{\prime} k}}+\frac{N_{5}^{\prime \prime} \widetilde{N_{5}^{\prime \prime}}}{t s_{q k}}+\frac{N_{7}^{\prime \prime} \widetilde{N_{7}^{\prime \prime}}}{t t^{\prime}}\right)
$$

that has the structure given in Eqs. (3.24) and (3.25). The calculation of the coefficients $\mathcal{A}_{i}$ in MRK gives

$$
\mathcal{A}_{p p}=\left(\alpha_{1}-2 \frac{\beta_{1}}{\beta_{2}}\right)^{2}+4 \alpha_{2} \beta_{1}\left(\frac{\alpha_{1}}{\beta_{2}^{2}}\right)+\ldots
$$




$$
\begin{aligned}
\mathcal{A}_{q q} & =\left(\beta_{2}+2 \frac{\alpha_{2}}{\alpha_{1}}\right)^{2}-4 \alpha_{2} \beta_{1}\left(\frac{\beta_{2}}{\alpha_{1}^{2}}\right)+\ldots, \\
\mathcal{A}_{p q} & =\left(\alpha_{1}-2 \frac{\beta_{1}}{\beta_{2}}\right)\left(\beta_{2}+2 \frac{\alpha_{2}}{\alpha_{1}}\right)+\ldots, \\
\mathcal{A}_{k p} & =-\left(\alpha_{1}-2 \frac{\beta_{1}}{\beta_{2}}\right)+\ldots, \\
\mathcal{A}_{k q} & =-\left(\beta_{2}+2 \frac{\alpha_{2}}{\alpha_{1}}\right)+\ldots
\end{aligned}
$$

Again, this correctly reproduces the part $\Omega^{\mu} \Omega^{\nu}$ of the MRK gravitational amplitude, but it falls short of reproducing the terms canceling the overlapping divergences.

To conclude our analysis, we would like to investigate which topologies in the original Feynman diagrams contribute to the different terms in the coefficients obtained in Eq. (3.27). To trace each contribution, we rescale the original numerators in Eq. (3.12) by some constants $D_{i}$ according to $N_{i} \rightarrow N_{i} D_{i}$ and study the expansion in the MRK regime. This results in the following form for the coefficients:

$$
\begin{aligned}
\mathcal{A}_{p p}= & \alpha_{1}^{2}-2\left(\frac{D_{1}+D_{2}}{D_{7}}\right) \frac{\alpha_{1} \beta_{1}}{\beta_{2}}+2\left(\frac{D_{1}^{2}+D_{2}^{2}}{D_{7}^{2}}\right) \frac{\beta_{1}^{2}}{\beta_{2}^{2}}+\left(\frac{D_{1}+D_{2}}{D_{7}}\right) \frac{\alpha_{2} \beta_{1} \alpha_{1}}{\beta_{2}^{2}} \\
- & {\left[\frac{D_{1}^{2}-3 D_{2} D_{1}+3 D_{4} D_{1}+2 D_{2}^{2}+3 D_{2} D_{4}-4\left(D_{1}+D_{2}\right) D_{7}}{D_{7}^{2}}\right] \frac{\alpha_{2} \beta_{1}}{\beta_{2}}+\ldots } \\
& \longrightarrow\left(\alpha_{1}-2 \frac{D_{b}}{D_{7}} \frac{\beta_{1}}{\beta_{2}}\right)^{2}+\frac{\alpha_{2} \beta_{1}\left[2 D_{7} D_{b} \alpha_{1}-\beta_{2}\left(6 D_{b}^{2}-8 D_{7} D_{b}\right)\right]}{D_{7}^{2} \beta_{2}^{2}} .
\end{aligned}
$$

Note that, in the last line, we have simplified the expression collecting similar topologies using the same constant for them, i.e. $D_{b} \equiv D_{1}=D_{2}=D_{4}=D_{5}$, where with the subscript $b$ indicates that they are associated with the diagrams where the gluon is emitted by bremsstrahlung from a scalar line. $D_{3,6}$ mark the diagrams containing the scalar-scalar-gluon-gluon vertex and $D_{7}$ the diagram 
with the three gluon vertex. The remaining results are

$$
\begin{aligned}
& \mathcal{A}_{q q}=\beta_{2}^{2}+2\left(\frac{D_{4}+D_{5}}{D_{7}}\right) \frac{\alpha_{2} \beta_{2}}{\alpha_{1}}+2\left(\frac{D_{4}^{2}+D_{5}^{2}}{D_{7}^{2}}\right) \frac{\alpha_{2}^{2}}{\alpha_{1}^{2}}-\left(\frac{D_{4}+D_{5}}{D_{7}}\right) \frac{\alpha_{2} \beta_{1} \beta_{2}}{\alpha_{1}^{2}}+\ldots \\
& +\left[\frac{D_{4}^{2}-3 D_{5} D_{4}+2 D_{5}^{2}+\left(D_{4}+D_{5}\right)\left(3 D_{1}-4 D_{7}\right)}{D_{7}^{2}}\right] \frac{\alpha_{2} \beta_{1}}{\alpha_{1}} \\
& \longrightarrow\left(\beta_{2}+2 \frac{D_{b}}{D_{7}} \frac{\alpha_{2}}{\alpha_{1}}\right)^{2}+\frac{\alpha_{2} \beta_{1}\left(\alpha_{1}\left(6 D_{b}^{2}-8 D_{7} D_{b}\right)-2 D_{7} \beta_{2} D_{b}\right)}{D_{7}^{2} \alpha_{1}^{2}}, \\
& \mathcal{A}_{p q}=\alpha_{1} \beta_{2}-\frac{\left(D_{1}+D_{2}\right)}{D_{7}} \beta_{1}+\frac{\left(D_{4}+D_{5}\right) \alpha_{2}}{D_{7}}-\frac{\left(D_{1}+D_{2}\right)\left(D_{4}+D_{5}\right)}{D_{7}^{2}} \frac{\beta_{1} \alpha_{2}}{\alpha_{1} \beta_{2}}+\ldots \\
& \longrightarrow\left(\alpha_{1}-2 \frac{D_{b}}{D_{7}} \frac{\beta_{1}}{\beta_{2}}\right)\left(\beta_{2}+2 \frac{D_{b}}{D_{7}} \frac{\alpha_{2}}{\alpha_{1}}\right) \\
& \mathcal{A}_{k p}=-\alpha_{1}+\frac{\left(D_{1}+D_{2}\right)}{D_{7}} \frac{\beta_{1}}{\beta_{2}}+\ldots \\
& \longrightarrow-\left(\alpha_{1}-2 \frac{D_{b}}{D_{7}} \frac{\beta_{1}}{\beta_{2}}\right) \\
& \mathcal{A}_{k q}=-\beta_{2}-\frac{\left(D_{4}+D_{5}\right)}{D_{7}} \frac{\alpha_{2}}{\alpha_{1}}+\ldots \\
& \longrightarrow \quad-\left(\beta_{2}+2 \frac{D_{b}}{D_{7}} \frac{\alpha_{2}}{\alpha_{1}}\right) \\
& \mathcal{A}_{k q}=-\beta_{2}-\frac{\left(D_{4}+D_{5}\right)}{D_{7}} \frac{\alpha_{2}}{\alpha_{1}}+\ldots \\
& \longrightarrow \quad-\left(\beta_{2}+2 \frac{D_{b}}{D_{7}} \frac{\alpha_{2}}{\alpha_{1}}\right) \text {. }
\end{aligned}
$$

It is remarkable that the MRK limit is blind to the constants $D_{3,6}$ and therefore 
to the diagrams with the scalar-scalar-gluon-gluon vertex.

We have investigated the color-kinematics duality proposed by Bern, Carrasco, and Johannson for the construction of gravitational amplitudes as a formal double-copy of equivalent amplitudes in gauge theories. In the gauge theory side we work with scalar QCD, and study the scattering of two distinct scalars with production of a gluon in the final state. We write the amplitude in two different representations and construct the corresponding "gravitational" scattering amplitudes using the BCJ doubling prescription. Despite them being different, when taking the multi-Regge limit of these amplitudes both BCJ representations reproduce the part of the MRK gravitational vertex (with two Reggeized gravitons and one on-shell graviton) which corresponds to the square of the MRK vertex in QCD (with two Reggeized gluons and one on-shell gluon). The subleading terms responsible for the fulfillment of the Steinmann conditions are not reproduced correctly and are dependent on the choice of numerators satisfying BCJ duality used to write the gauge amplitude. This has a likely origin in the external matter states of the gauge amplitudes, for which the duality does not hold. 


\section{Chapter 4}

\section{BCJ and Dimensional Reduction}

\subsection{Solving the puzzle}

In the previous chapter, we studied color-kinematics duality in the context of inelastic amplitudes involving scalar particles in multi-Regge kinematics (the relation between multi-Regge kinematics [64, 65] and supergravity amplitudes in the BCJ context has been explored in [60]). We showed that an initial application of the BCJ duality to the scattering of two scalar particles with gluon emission in scalar QCD only retrieves part of the gravitational amplitude. More precisely, the part that was correctly obtained corresponds to the square of two Lipatov's QCD emission vertices [20, 21, 22]. The terms crucial for the cancellation of simultaneous divergences in overlapping channels [25, 23, 28, 29, 60, 24], as required by unitarity (Steinmann relations [57]), were absent in this application of the duality.

While the main line of studies of color-kinematics duality deals with pure (super)-Yang-Mills theories in various dimensions, where the double-copy prescription is proven [38], the calculation presented in the previous chapter incorporated additional minimally-coupled matter states in Yang-Mills theory. As this 
is a setup that is outside of the standard application of color-kinematics duality, it is perhaps not unexpected that a refined double-copy prescription is needed. Our aim in the present chapter is to study this problem from a new point of view, approaching it with two different modifications [66]. Firstly, we consider the scattering of two distinguishable scalars in Yang-Mills theory, where the scalars live in the adjoint representation. As a crucial new element, we introduce the quartic matter self-coupling characteristic of the bosonic sector of $\mathcal{N}=2$ supersymmetric Yang-Mills theory. Secondly, we repeat the calculation of Section 3.2, only this time considering identical adjoint scalars. In both cases, the color-kinematics duality reproduces, in the Regge limit, the gravitational amplitude as computed in Chapter 2, including those terms responsible for the fulfillment of the Steinmann relations. The $D=4$ Yang-Mills + scalar theories studied here are via dimensional reduction directly related to pure Yang-Mills theory in $D=6$ and $D=5$ dimensions, respectively. Similarly, the theories are the bosonic sectors of the $\mathcal{N}=2$ and $\mathcal{N}=1$ super-Yang-Mills theories. This explains why the inclusion of matter in these cases is straightforward from the perspective of color-kinematics duality.

\subsection{Color-kinematics duality with scalar matter}

We study gauge-theory scattering of two scalar particles with the emission of a gluon; and later on, gravity scattering of two scalars with the emission of a graviton. These have momenta $p_{1}, p_{2}$ (incoming scalars), $p_{3}, p_{4}$ (outgoing scalars), and $p_{5}$ (emitted gluon/graviton), which are all taken to enter the diagram. Before particularizing to the case of distinguishable or indistinguishable scalars and matter self-interactions, we carry out a general analysis.

After resolving any quartic vertices into trivalent ones, the five-point gauge- 
theory amplitude can be written as a sum over 15 channels ${ }^{1}$

$$
\mathcal{A}_{5}=g^{3} \sum_{i=1}^{15} \frac{c_{i} n_{i}}{d_{i}}
$$

where $c_{i}$ are the color factors defined by

$$
\begin{array}{ll}
c_{1}=f^{a_{5} a_{3} b} f^{b a_{4} c} f^{c a_{2} a_{1}}, & c_{2}=f^{a_{5} a_{4} b} f^{b a_{3} c} f^{c a_{2} a_{1}}, \\
c_{3}=f^{a_{2} a_{1} b} f^{b a_{5} c} f^{c a_{3} a_{4}}, & c_{4}=f^{a_{5} a_{1} b} f^{b a_{2} c} f^{c a_{3} a_{4}}, \\
c_{5}=f^{a_{5} a_{2} c} f^{c a_{1} b} f^{b a_{3} a_{4}}, & c_{6}=f^{a_{5} a_{3} c} f^{c a_{1} b} f^{b a_{2} a_{4}}, \\
c_{7}=f^{a_{5} a_{4} b} f^{b a_{2} c} f^{c a_{3} a_{1}}, & c_{8}=f^{a_{5} a_{4} c} f^{c a_{1} b} f^{b a_{2} a_{3}}, \\
c_{9}=f^{a_{5} a_{3} b} f^{b a_{2} c} f^{c a_{4} a_{1}}, & c_{10}=f^{a_{5} a_{1} b} f^{b a_{3} c} f^{c a_{2} a_{4}}, \\
c_{11}=f^{a_{5} a_{2} b} f^{b a_{4} c} f^{c a_{3} a_{1}}, & c_{12}=f^{a_{5} a_{2} b} f^{b a_{3} c} f^{c a_{4} a_{1},}, \\
c_{13}=f^{a_{5} a_{1} b} f^{b a_{4} c} f^{c a_{2} a_{3}}, & c_{14}=f^{a_{2} a_{4} b} f^{b a_{5} c} f^{c a_{3} a_{1}}, \\
c_{15}=f^{a_{2} a_{3} b} f^{b a_{5} c} f^{c a_{4} a_{1}}, &
\end{array}
$$

with $f^{a b c}$ being the structure constants. Finally, the denominators

$$
d_{i}=\prod_{\alpha_{i}} s_{\alpha_{i}}
$$

correspond to the product of the kinematic invariants associated with the internal lines in the $i$-th diagram (the numbering is the one shown in Fig. 4.1).

Due to the Jacobi identities of the structure constants, the color factors satisfy nine independent identities that we label as $j_{\alpha}$. They are

\footnotetext{
${ }^{1}$ Since, unlike in the previous chapter, we are considering here that all states are in the adjoint representation, and all diagrams are trivalent, we revert to the notation of Chapter 1 . where both color factors and numerators are denoted by lower case letters.
} 

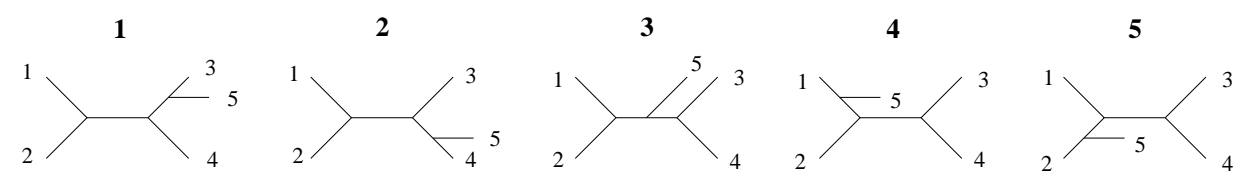

6

7

8

9

10
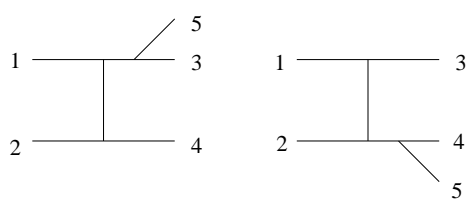

12

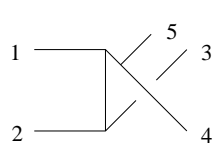

13
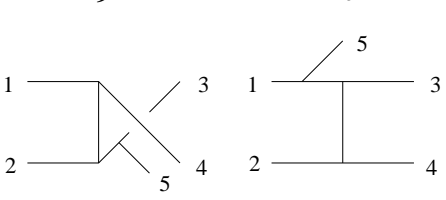

11

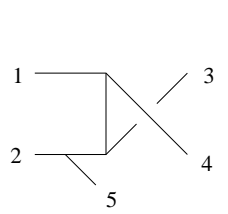

14

15
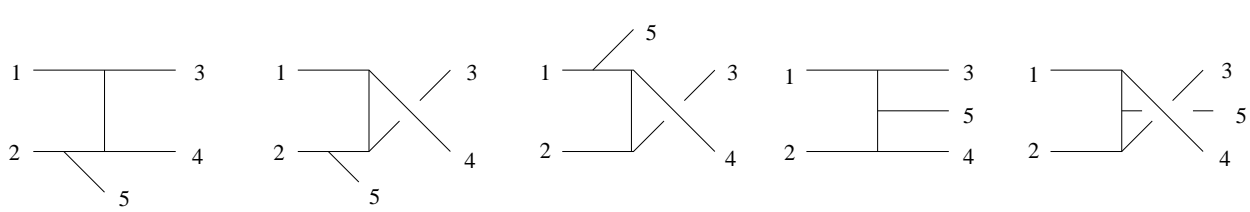

Figure 4.1: The fifteen three-vertex topologies contributing to the sum in the amplitude (4.1). The labels 1-4 correspond to the scalars and 5 to the gluon.

$$
\begin{aligned}
& j_{1} \equiv c_{12}-c_{9}+c_{15}=0, \\
& j_{2} \equiv c_{11}-c_{7}+c_{14}=0, \\
& j_{3} \equiv-c_{4}+c_{5}+c_{3}=0, \\
& j_{4} \equiv c_{1}-c_{2}-c_{3}=0, \\
& j_{5} \equiv-c_{10}+c_{6}-c_{14}=0, \\
& j_{6} \equiv-c_{13}+c_{8}-c_{15}=0, \\
& j_{7} \equiv c_{4}-c_{10}+c_{13}=0, \\
& j_{8} \equiv c_{8}+c_{7}-c_{2}=0, \\
& j_{9} \equiv c_{6}+c_{9}-c_{1}=0 .
\end{aligned}
$$

The numerators $n_{i}$ in Eq. (4.1) can be computed from the Feynman rules of the theory. In general, they will not satisfy the Jacobi-like identities $\pm n_{i} \pm n_{j} \pm$ 
$n_{k}=0$, corresponding to $j_{\alpha}$ with $c_{i} \rightarrow n_{i}$. However, this can be cured after performing a generalized gauge transformation, consisting of adding zero to the original amplitude in the form

$$
\mathcal{A}_{5}=\sum_{i=1}^{15} \frac{c_{i} n_{i}}{d_{i}}+\sum_{\alpha=1}^{9} \gamma_{\alpha} j_{\alpha}=\sum_{i=1}^{15} \frac{c_{i} n_{i}^{\prime}}{d_{i}}
$$

where the new numerators $n_{i}^{\prime}$ are obtained by collecting the coefficients of each color factor $c_{i}$ and multiplying by corresponding denominator: $n_{i}^{\prime}=d_{i} \partial_{c_{i}} \mathcal{A}_{5}$. The parameters $\gamma_{\alpha}$ are unknown functions of the momenta and gluon polarization. They are determined by forcing the new numerators to satisfy the Jacobi identities

$$
\left.j_{\alpha}\right|_{c_{i} \rightarrow n_{i}^{\prime}}=0
$$

These new numerators will be used to construct the corresponding gravitational amplitude using the BCJ double-copy prescription

$$
-i \mathcal{M}=\left(\frac{\kappa}{2}\right)^{3} \sum_{i=1}^{15} \frac{n_{i}^{\prime} \widetilde{n}_{i}^{\prime}}{d_{i}}
$$

with $\kappa$ being the gravitational coupling constant.

As usual we express the momenta in terms of Sudakov parameters. As a first step we write

$$
p_{3}=-p_{1}+k_{1}, \quad p_{4}=-p_{2}-k_{2}, \quad p_{5}=-k_{1}+k_{2},
$$

where in turn $k_{1}$ and $k_{2}$ are written as

$$
k_{1}^{\mu}=\alpha_{1} p_{1}^{\mu}+\beta_{1} p_{2}^{\mu}+k_{1, \perp}^{\mu}, \quad k_{2}^{\mu}=\alpha_{2} p_{1}^{\mu}+\beta_{2} p_{2}^{\mu}+k_{2, \perp}^{\mu},
$$


with $k_{i, \perp}$ being vectors orthogonal to $p_{1}$ and $p_{2}$. Then, the gluon momentum takes the form

$$
p_{5}^{\mu}=\left(\alpha_{2}-\alpha_{1}\right) p_{1}^{\mu}+\left(\beta_{2}-\beta_{1}\right) p_{2}^{\mu}+k_{2, \perp}^{\mu}-k_{1, \perp}^{\mu} .
$$

Finally, the multi-Regge kinematics regime is defined in terms of the Sudakov parameters by

$$
1 \gg \alpha_{1} \gg \alpha_{2}, \quad 1 \gg\left|\beta_{1}\right| \gg\left|\beta_{2}\right|
$$

As in Chapter 2, the gravitational amplitude (4.7) can then be written as

$$
-i \mathcal{M}=-i A_{k k} \mathcal{M}^{\mu \nu} \epsilon_{\mu \nu}\left(p_{5}\right),
$$

where $\epsilon_{\mu \nu}\left(p_{5}\right)$ is the graviton polarization tensor

$$
\begin{aligned}
\mathcal{M}^{\mu \nu}= & \left(k_{1}+k_{2}\right)_{\perp}^{\mu}\left(k_{1}+k_{2}\right)_{\perp}^{\nu}+\mathcal{A}_{k 1}\left[\left(k_{1}+k_{2}\right)_{\perp}^{\mu} p_{1}^{\nu}+p_{1}^{\mu}\left(k_{1}+k_{2}\right)_{\perp}^{\nu}\right] \\
& +\mathcal{A}_{k 2}\left[\left(k_{1}+k_{2}\right)_{\perp}^{\mu} p_{2}^{\nu}+p_{2}^{\mu}\left(k_{1}+k_{2}\right)_{\perp}^{\nu}\right]+\mathcal{A}_{12}\left(p_{1}^{\mu} p_{2}^{\nu}+p_{2}^{\mu} p_{1}^{\nu}\right) \\
& +\mathcal{A}_{11} p_{1}^{\mu} p_{1}^{\nu}+\mathcal{A}_{22} p_{2}^{\mu} p_{2}^{\nu}
\end{aligned}
$$

The parameters $\mathcal{A}_{k 1}, \mathcal{A}_{k 2}, \mathcal{A}_{12}, \mathcal{A}_{11}, \mathcal{A}_{22}$ are to be determined. The coefficient $A_{k k}$ contains all the information about the coupling to external particles in the amplitude. By factoring it out we isolate Lipatov's graviton emission effective vertex $\mathcal{M}^{\mu \nu}$, which in multi-Regge kinematics is independent of the states involved in the collision and has the structure shown in Eqs. (3.29)-3.31). 


\subsubsection{Distinguishable scalars.}

We first deal with the scattering of two distinguishable scalars $\Phi$ and $\Phi^{\prime}$. In Chapter 3 we have analyzed this problem and found that the BCJ prescription only reproduces the QCD-like part of the gravitational amplitudes; that is, the $\Omega^{\mu} \Omega^{\nu}$ piece in Eq. 3.29 . There we noted that the problem with the incorrect $\mathcal{N}^{\mu} \mathcal{N}^{\nu}$ term is solved by embedding the Yang-Mills +2 scalar theory into the bosonic sector of $\mathcal{N}=2 \mathrm{SYM}$ theory, which amounts to taking both scalars to transform in the adjoint representation and introducing a matter self-coupling for the two scalars of the form

$$
\Delta \mathcal{L}=\frac{g^{2}}{2} \operatorname{Tr}\left(\left[\Phi, \Phi^{\prime}\right]^{2}\right)
$$

The corresponding Feynman rules then contain a new quartic scalar contact vertex and we need to add four more diagrams to the ones shown in (3.7). Evaluating all contributions, we find the following values for the numerators $n_{i}^{\prime}$

$$
\begin{aligned}
n_{1}^{\prime}= & \left(p_{1}+p_{2}\right)^{2}\left[-\left(\gamma_{9}-\gamma_{4}\right)\left(p_{3}+p_{5}\right)^{2}-2 p_{3} \cdot \epsilon\left(p_{5}\right)\right], \\
n_{2}^{\prime}= & \left(p_{1}+p_{2}\right)^{2}\left[-\left(\gamma_{4}+\gamma_{8}\right)\left(p_{4}+p_{5}\right)^{2}+2 p_{4} \cdot \epsilon\left(p_{5}\right)\right], \\
n_{3}^{\prime}= & \left(\gamma_{3}-\gamma_{4}\right)\left(p_{1}+p_{2}\right)^{2}\left(p_{3}+p_{4}\right)^{2}, \\
n_{4}^{\prime}= & \left(p_{3}+p_{4}\right)^{2}\left[\left(\gamma_{7}-\gamma_{3}\right)\left(p_{1}+p_{5}\right)^{2}+2 p_{1} \cdot \epsilon\left(p_{5}\right)\right], \\
n_{5}^{\prime}= & -\left(p_{3}+p_{4}\right)^{2}\left[-\gamma_{3}\left(p_{2}+p_{5}\right)^{2}+2 p_{2} \cdot \epsilon\left(p_{5}\right)\right], \\
n_{6}^{\prime}= & -\left(p_{3}+p_{5}\right)^{2}\left[-\left(\gamma_{5}+\gamma_{9}\right)\left(p_{2}+p_{4}\right)^{2}+\left(p_{2}-p_{4}\right) \cdot \epsilon\left(p_{5}\right)\right] \\
& -2\left(p_{2}-p_{4}\right) \cdot\left(p_{1}-p_{3}-p_{5}\right)\left[p_{3} \cdot \epsilon\left(p_{5}\right)\right], \\
n_{7}^{\prime}= & -\left(p_{4}+p_{5}\right)^{2}\left[\left(\gamma_{2}-\gamma_{8}\right)\left(p_{1}+p_{3}\right)^{2}+\left(p_{3}-p_{1}\right) \cdot \epsilon\left(p_{5}\right)\right] \\
& -2\left(p_{3}-p_{1}\right) \cdot\left(p_{2}-p_{4}-p_{5}\right)\left[p_{3} \cdot \epsilon\left(p_{5}\right)\right],
\end{aligned}
$$




$$
\begin{aligned}
n_{8}^{\prime}= & \left(p_{2}+p_{3}\right)^{2}\left[\left(\gamma_{6}+\gamma_{8}\right)\left(p_{4}+p_{5}\right)^{2}+2 p_{4} \cdot \epsilon\left(p_{5}\right)\right], \\
n_{9}^{\prime}= & -\left(p_{1}+p_{4}\right)^{2}\left[\left(\gamma_{1}-\gamma_{9}\right)\left(p_{3}+p_{5}\right)^{2}+2 p_{3} \cdot \epsilon\left(p_{5}\right)\right], \\
n_{10}^{\prime}= & -\left(p_{1}+p_{5}\right)^{2}\left[\left(\gamma_{5}+\gamma_{7}\right)\left(p_{2}+p_{4}\right)^{2}+\left(p_{2}-p_{4}\right) \cdot \epsilon\left(p_{5}\right)\right] \\
& -2\left(p_{2}-p_{4}\right) \cdot\left(-p_{1}+p_{3}-p_{5}\right)\left[p_{1} \cdot \epsilon\left(p_{5}\right)\right], \\
n_{11}^{\prime}= & -\left(p_{2}+p_{5}\right)^{2}\left[-\gamma_{2}\left(p_{1}+p_{3}\right)^{2}+\left(p_{3}-p_{1}\right) \cdot \epsilon\left(p_{5}\right)\right] \\
& -2\left(p_{3}-p_{1}\right) \cdot\left(-p_{2}+p_{4}-p_{5}\right)\left[p_{2} \cdot \epsilon\left(p_{5}\right)\right], \\
n_{12}^{\prime}= & \left(p_{1}+p_{4}\right)^{2}\left[\gamma_{1}\left(p_{2}+p_{5}\right)^{2}+2 p_{2} \cdot \epsilon\left(p_{5}\right)\right], \\
n_{13}^{\prime}= & -\left(p_{2}+p_{3}\right)^{2}\left[\left(\gamma_{6}-\gamma_{7}\right)\left(p_{1}+p_{5}\right)^{2}+2 p_{1} \cdot \epsilon\left(p_{5}\right)\right], \\
n_{14}^{\prime}= & -\left(p_{2}-p_{4}\right) \cdot\left(p_{1}+p_{3}-p_{5}\right)\left[\left(p_{3}-p_{1}\right) \cdot \epsilon\left(p_{5}\right)\right] \\
& -\left(p_{3}-p_{1}\right) \cdot\left(p_{2}-p_{4}\right)\left[\left(-p_{1}+p_{2}-p_{3}+p_{4}\right) \cdot \epsilon\left(p_{5}\right)\right] \\
& +\gamma_{2}\left(p_{1}+p_{3}\right)^{2}\left(p_{2}+p_{4}\right)^{2}-\gamma_{5}\left(p_{1}+p_{3}\right)^{2}\left(p_{2}+p_{4}\right)^{2}, \\
n_{15}^{\prime}= & \left(\gamma_{1}-\gamma_{6}\right)\left(p_{2}+p_{3}\right)^{2}\left(p_{1}+p_{4}\right)^{2} .
\end{aligned}
$$

Although, in principle, we have nine equations for the nine functions $\gamma_{\alpha}$, momentum conservation makes some of the nine conditions in Eq. (4.6) linearly dependent. One convenient way to implement momentum conservation is to express our momenta using the Sudakov parameters introduced above. Doing so, we find that the equation system is described by a $9 \times 9$ matrix that has rank 5 and the solution can be written in terms of 4 independent variables that we take to be $\gamma_{1}, \gamma_{3}, \gamma_{6}$ and $\gamma_{7}$ :

$$
\begin{aligned}
& \gamma_{2}=\frac{\left(p_{2}+2 p_{3}+p_{4}\right) \cdot \epsilon\left(p_{5}\right)}{s \beta_{1}}-\gamma_{1} \frac{1+\beta_{2}}{\beta_{1}}-\gamma_{3} \frac{-1+\alpha_{1}-\alpha_{2}+\beta_{1}-\beta_{2}}{\beta_{1}} \\
& \gamma_{4}=\frac{2\left(p_{3}+p_{4}\right) \cdot \epsilon\left(p_{5}\right)}{s}+\gamma_{3}\left(1-\alpha_{1}+\alpha_{2}-\beta_{1}+\beta_{2}\right)+\gamma_{7}\left(\beta_{1}-\beta_{2}\right), \\
& \gamma_{5}=\frac{\left(-p_{2}+p_{4}\right) \cdot \epsilon\left(p_{5}\right)}{s \alpha_{2}}-\gamma_{3} \frac{1-\alpha_{1}+\alpha_{2}-\beta_{1}+\beta_{2}}{\alpha_{2}}+\gamma_{6} \frac{1-\alpha_{1}}{\alpha_{2}}-\gamma_{7} \frac{\beta_{1}-\beta_{2}}{\alpha_{2}},
\end{aligned}
$$




$$
\begin{aligned}
\gamma_{8} & =\frac{2\left(p_{2}+p_{3}\right) \cdot \epsilon\left(p_{5}\right)}{s\left(\alpha_{1}+\beta_{1}\right)}-\gamma_{1} \frac{1+\beta_{2}}{\alpha_{1}+\beta_{1}}+\gamma_{6} \frac{1-\alpha_{1}}{\alpha_{1}+\beta_{1}}-\gamma_{7} \frac{\beta_{1}-\beta_{2}}{\alpha_{1}+\beta_{1}}, \\
\gamma_{9} & =-\frac{2\left(p_{2}+p_{3}\right) \cdot \epsilon\left(p_{5}\right)}{s\left(\alpha_{2}+\beta_{2}\right)}+\gamma_{1} \frac{1+\beta_{2}}{\alpha_{2}+\beta_{2}}-\gamma_{6} \frac{1-\alpha_{1}}{\alpha_{2}+\beta_{2}} .
\end{aligned}
$$

After applying the BCJ prescription (4.7), the four independent $\gamma$ 's cancel out of the gravitational amplitude, so we set them to zero from now on. Plugging the five remaining $\gamma$ 's back in the numerators of Eq. 4.15), we construct the gravitational amplitude from Eq. 4.7). In the multi-Regge kinematics limit, the coefficients in Eq. (4.13) take the form

$$
\begin{aligned}
& \mathcal{A}_{11} \simeq \alpha_{1}^{2}-\frac{4 \alpha_{1} \beta_{1}}{\beta_{2}}+\frac{4 \beta_{1}^{2}}{\beta_{2}^{2}}+\frac{4 \alpha_{2} \beta_{1}}{\beta_{2}^{2}}+\ldots, \\
& \mathcal{A}_{22} \simeq \beta_{2}^{2}+\frac{4 \alpha_{2} \beta_{1}}{\alpha_{1}}+\frac{4 \alpha_{2} \beta_{1}}{\alpha_{1}^{2}}+\frac{4 \alpha_{2}^{2}}{\alpha_{1}^{2}}+\ldots, \\
& \mathcal{A}_{12} \simeq \alpha_{1} \beta_{2}-2 \beta_{1}+2 \alpha_{2}+\ldots, \\
& \mathcal{A}_{k 1} \simeq-\alpha_{1}+\frac{2 \beta_{1}}{\beta_{2}}+\ldots, \\
& \mathcal{A}_{k 2} \simeq-\beta_{2}-\frac{2 \alpha_{2}}{\alpha_{1}}+\ldots,
\end{aligned}
$$

where the ellipsis denote subleading contributions in the multi-Regge limit. The above coefficients correctly reproduce the full form of Lipatov's effective graviton emission vertex shown in Eq. (3.29), including the $\mathcal{N}^{\mu} \mathcal{N}^{\nu}$ piece that was not correctly retrieved in the analysis of the previous chapter.

We note that away from the Regge-limit the amplitude obtained from Eq. (4.7) is still a valid gravitational amplitude, however, it includes one additional contact term of the form

$$
\Delta \mathcal{L} \sim \kappa^{2} g^{\mu \nu} \Phi^{\prime 2} \partial_{\mu} \partial_{\nu} \Phi^{2}
$$

This term typically appears in the bosonic sector of SUGRA theories, or in di- 
mensional reductions of $D>4$ gravities. If desired, this contribution can be subtracted from the amplitude, thus obtaining a gravity amplitude with minimally coupled scalars.

\subsubsection{Indistinguishable scalars.}

A second possibility to amend the results of Chapter 3 is to consider two indistinguishable scalars transforming in the adjoint representation of the gauge group. Again, the number of Feynman diagrams contributing to the amplitude is larger than the original calculation, since all channels are allowed. Resolving the diagrams containing four-leg vertices in terms of trivalent ones, we arrive at the following form of the numerators in Eq. 4.1):

$$
\begin{aligned}
n_{1}= & -\left(p_{3}+p_{5}\right)^{2}\left[\left(p_{2}-p_{1}\right) \cdot \epsilon\left(p_{5}\right)\right]-2\left(p_{2}-p_{1}\right) \cdot\left(-p_{3}+p_{4}-p_{5}\right)\left[p_{3} \cdot \epsilon\left(p_{5}\right)\right], \\
n_{2}= & -\left(p_{4}+p_{5}\right)^{2}\left[\left(p_{2}-p_{1}\right) \cdot \epsilon\left(p_{5}\right)\right]-2\left(p_{2}-p_{1}\right) \cdot\left(p_{3}-p_{4}-p_{5}\right)\left[p_{4} \cdot \epsilon\left(p_{5}\right)\right], \\
n_{3}= & -\left(p_{2}-p_{1}\right) \cdot\left(p_{3}-p_{4}\right)\left[\left(p_{1}+p_{2}-p_{3}-p_{4}\right) \cdot \epsilon\left(p_{5}\right)\right] \\
& -\left(p_{3}-p_{4}\right) \cdot\left(-p_{1}-p_{2}+p_{5}\right)\left[\left(p_{2}-p_{1}\right) \cdot \epsilon\left(p_{5}\right)\right] \\
& -\left(p_{2}-p_{1}\right) \cdot\left(p_{3}+p_{4}-p_{5}\right)\left[\left(p_{3}-p_{4}\right) \cdot \epsilon\left(p_{5}\right)\right], \\
n_{4}= & -\left(p_{1}+p_{5}\right)^{2}\left[\left(p_{3}-p_{4}\right) \cdot \epsilon\left(p_{5}\right)\right]-2\left(p_{3}-p_{4}\right) \cdot\left(-p_{1}+p_{2}-p_{5}\right)\left[p_{1} \cdot \epsilon\left(p_{5}\right)\right], \\
n_{5}= & -\left(p_{2}+p_{5}\right)^{2}\left[\left(p_{3}-p_{1}\right) \cdot \epsilon\left(p_{5}\right)\right]-2\left(p_{3}-p_{1}\right) \cdot\left(-p_{2}+p_{4}-p_{5}\right)\left[p_{2} \cdot \epsilon\left(p_{5}\right)\right], \\
n_{6}= & -\left(p_{3}+p_{5}\right)^{2}\left[\left(p_{4}-p_{1}\right) \cdot \epsilon\left(p_{5}\right)\right]-2\left(p_{2}-p_{1}\right) \cdot\left(-p_{3}+p_{4}-p_{5}\right)\left[p_{3} \cdot \epsilon\left(p_{5}\right)\right], \\
n_{7}= & -\left(p_{4}+p_{5}\right)^{2}\left[\left(p_{3}-p_{1}\right) \cdot \epsilon\left(p_{5}\right)\right]-2\left(p_{3}-p_{1}\right) \cdot\left(p_{2}-p_{4}-p_{5}\right)\left[p_{4} \cdot \epsilon\left(p_{5}\right)\right], \\
n_{8}= & -\left(p_{4}+p_{5}\right)^{2}\left[\left(p_{2}-p_{3}\right) \cdot \epsilon\left(p_{5}\right)\right]-2\left(p_{2}-p_{3}\right) \cdot\left(p_{1}-p_{4}-p_{5}\right)\left[p_{4} \cdot \epsilon\left(p_{5}\right)\right], \\
n_{9}= & -\left(p_{3}+p_{5}\right)^{2}\left[\left(p_{4}-p_{1}\right) \cdot \epsilon\left(p_{5}\right)\right]-2\left(p_{4}-p_{1}\right) \cdot\left(p_{2}-p_{3}-p_{5}\right)\left[p_{3} \cdot \epsilon\left(p_{5}\right)\right], \\
n_{10}= & -\left(p_{1}+p_{5}\right)^{2}\left[\left(p_{2}-p_{4}\right) \cdot \epsilon\left(p_{5}\right)\right]-2\left(p_{2}-p_{4}\right) \cdot\left(-p_{1}+p_{3}-p_{5}\right)\left[p_{1} \cdot \epsilon\left(p_{5}\right)\right], \\
n_{11}= & -\left(p_{2}+p_{5}\right)^{2}\left[\left(p_{3}-p_{1}\right) \cdot \epsilon\left(p_{5}\right)\right]-2\left(p_{3}-p_{1}\right) \cdot\left(-p_{2}+p_{4}-p_{5}\right)\left[p_{2} \cdot \epsilon\left(p_{5}\right)\right],
\end{aligned}
$$




$$
\begin{aligned}
n_{12}= & -\left(p_{2}+p_{5}\right)^{2}\left[\left(p_{4}-p_{1}\right) \cdot \epsilon\left(p_{5}\right)\right]-2\left(p_{4}-p_{1}\right) \cdot\left(-p_{2}+p_{3}-p_{5}\right)\left[p_{2} \cdot \epsilon\left(p_{5}\right)\right], \\
n_{13}= & -\left(p_{1}+p_{5}\right)^{2}\left[\left(p_{2}-p_{3}\right) \cdot \epsilon\left(p_{5}\right)\right]-2\left(p_{2}-p_{3}\right) \cdot\left(-p_{1}+p_{4}-p_{5}\right)\left[p_{1} \cdot \epsilon\left(p_{5}\right)\right], \\
n_{14}= & -\left(p_{2}-p_{4}\right) \cdot\left(p_{1}+p_{3}-p_{5}\right)\left[\left(p_{3}-p_{1}\right) \cdot \epsilon\left(p_{5}\right)\right] \\
& -\left(p_{3}-p_{1}\right) \cdot\left(-p_{2}-p_{4}+p_{5}\right)\left[\left(p_{2}-p_{4}\right) \cdot \epsilon\left(p_{5}\right)\right] \\
& -\left(p_{3}-p_{1}\right) \cdot\left(p_{2}-p_{4}\right)\left[\left(-p_{1}+p_{2}-p_{3}+p_{4}\right) \cdot \epsilon\left(p_{5}\right)\right] \\
n_{15}= & -\left(p_{2}-p_{3}\right) \cdot\left(p_{4}-p_{1}\right)\left[\left(-p_{1}+p_{2}+p_{3}-p_{4}\right) \cdot \epsilon\left(p_{5}\right)\right] \\
& -\left(p_{4}-p_{1}\right) \cdot\left(-p_{2}-p_{3}+p_{5}\right)\left[\left(p_{2}-p_{3}\right) \cdot \epsilon\left(p_{5}\right)\right] \\
& -\left(p_{2}-p_{3}\right) \cdot\left(p_{1}+p_{4}-p_{5}\right)\left[\left(p_{4}-p_{1}\right) \cdot \epsilon\left(p_{5}\right)\right] .
\end{aligned}
$$

Remarkably, these numerators obtained from the application of the Feynman rules automatically satisfy the Jacobi-like identities (4.6), so there is no need to perform a generalized gauge transformation. We immediately proceed to construct the gravitational amplitude using the BCJ prescription 4.7). After taking the multi-Regge kinematics limit 4.11 we find the following value for the coefficients in Eq. 4.13

$$
\begin{aligned}
& \mathcal{A}_{11} \simeq \alpha_{1}^{2}-\frac{4 \alpha_{1} \beta_{1}}{\beta_{2}}+\frac{4 \beta_{1}^{2}}{\beta_{2}^{2}}+\frac{4 \alpha_{2} \beta_{1}}{\beta_{2}^{2}}+\ldots \\
& \mathcal{A}_{22} \simeq \beta_{2}^{2}+\frac{4 \alpha_{2} \beta_{1}}{\alpha_{1}}+\frac{4 \alpha_{2} \beta_{1}}{\alpha_{1}^{2}}+\frac{4 \alpha_{2}^{2}}{\alpha_{1}^{2}}+\ldots \\
& \mathcal{A}_{12} \simeq \alpha_{1} \beta_{2}-2 \beta_{1}+2 \alpha_{2}+\ldots \\
& \mathcal{A}_{k 1} \simeq-\alpha_{1}+\frac{2 \beta_{1}}{\beta_{2}}+\ldots \\
& \mathcal{A}_{k 2} \simeq-\beta_{2}-\frac{2 \alpha_{2}}{\alpha_{1}}+\ldots
\end{aligned}
$$

which again reproduce Lipatov's effective vertex (3.29). 


\subsection{The role of dimensional reduction}

In this note we have addressed the problem of applying color-kinematics duality to the scattering of two distinguishable scalar matter particles with gluon emission, or graviton emission. The calculation in Chapter 3 suggested that the BCJ double-copy prescription only reproduced part of the gravitational amplitude in multi-Regge kinematics. We have found two ways to remedy this mismatch. One consists of introducing a contact interaction between the two scalar particles, as suggested by the bosonic sector of $\mathcal{N}=2 \mathrm{SYM}$ theory. The second is to give up distinguishability of the scalars. In both cases the introduction of new diagrams contributing to the process recovers the correct gravity amplitudes from the BCJ double-copy prescription, in the Regge limit.

The two cases can be thought of as the bosonic sectors of $\mathcal{N}=2$ and $\mathcal{N}=1$ SYM theory. Since fermions do not play any role in the tree-level amplitudes studied here, the obtained results can be fully explained by supersymmetry. However, we note that supersymmetry is not a mandatory explanation of the results, nor is it the most elegant one.

The interaction term 4.14 is generated by dimensionally reducing $D=6$ pure Yang-Mills to $D=4$, where the gauge field along the extra two dimensions are interpreted as two scalars, $\Phi \equiv A_{4}, \Phi^{\prime} \equiv A_{5}$. The additional components of the gauge field strength tensor are

$$
F_{\mu 4}=D_{\mu} \Phi, \quad F_{\mu 5}=D_{\mu} \Phi^{\prime}, \quad F_{45}=-i g\left[\Phi, \Phi^{\prime}\right],
$$

with $D_{\mu}$ being the adjoint covariant derivative. The four-dimensional Lagrangian 
is then

$$
\mathcal{L}=-\frac{1}{4} \operatorname{Tr}\left(F_{\mu \nu} F^{\mu \nu}\right)+\frac{1}{2} \operatorname{Tr}\left(D_{\mu} \Phi D^{\mu} \Phi\right)+\frac{1}{2} \operatorname{Tr}\left(D_{\mu} \Phi^{\prime} D^{\mu} \Phi^{\prime}\right)+\frac{g^{2}}{2} \operatorname{Tr}\left(\left[\Phi, \Phi^{\prime}\right]^{2}\right) .
$$

Similarly, the tree-level scattering of two indistinguishable scalars with gluon emission can be computed either from this Lagrangian or from the dimensional reduction of $D=5$ pure Yang-Mills to $D=4$ dimensions, which results in YangMills theory with one adjoint scalar, where $\Phi \equiv A_{4}$. In the latter case there is no quartic scalar term.

As a final remark, one can say that the successful application of color-kinematics duality in the cases studied here directly stems from its validity in higher-dimensional Yang-Mills theory and gravity [35, 67, 38, 68, 69, 70, 71]. Indeed, the dimensional reduction that we have employed here has been used in loop calculations in gauge and gravity theories with extended supersymmetry [72].

While the modified theories we have considered avoid the specific problem observed in Chapter 3, the inclusion of general matter states and interactions in the color-kinematics formalism is still an open problem [73]. Specifically, it would be interesting to understand how to precisely relate tree amplitudes in YangMills theory with minimally-coupled matter, $N_{s}$ scalars and $N_{f}$ fermions, to that of Einstein gravity with similar matter content. We expect that an extension of the BCJ prescription is needed, which at intermediate steps embed the gauge and gravity theories into their respective higher-dimensional versions. The results presented here, with the help of the information gained by taking the multi-Regge 
limit, were a first step towards understanding the general matter case. 


\section{Chapter 5}

\section{Conclusions and Outlook}

The aim of this thesis has been to gain a deeper understanding of gravity as a double copy of gauge theories at high energies, specifically in multi-Regge kinematics. We have confirmed that the Regge limit of a gravity theory coupled to scalars has the expected double copy structure $\Omega^{\mu} \Omega^{\nu}-\mathcal{N}^{\mu} \mathcal{N}^{\nu}$ which is further evidence that the Regge Limit in gravity is independent of the external scattered particles at either end of the ladder diagrams that dominate interactions in this limit. We have further written down the simplest form to date for the three-graviton vertex and found exact gauge invariant effective vertices in this amplitude that could be used to efficiently generate higher loop amplitudes in gravity, drastically cutting down the number of topologies.

We later attempted to introduce BCJ into the picture. This was motivated by the relation between BCJ, the double copy and the multi-Regge Limit. We confirmed in Chapter 2 that the multi-Regge limit of a gravitational amplitude coupled to scalars has a double copy-like form, with the added twist that the quantity $\mathcal{N}^{\mu} \mathcal{N}^{\nu}$ above is difficult to identify in YM amplitudes.

We found that naive attempts to apply BCJ to scalar QCD amplitudes only recover the $\Omega^{\mu} \Omega^{\nu}$ term in the Regge limit, which is to be expected given that 
we are essentially squaring the term that dominates the gauge theory amplitude. This a strong indication that these naive double copies are not indeed gravity amplitudes.

Initially guided by supersymmetry we carefully fine tune the scalar QCD theory we choose to apply BCJ to. Using this insight, we do manage to find examples of amplitudes that have non-trivial solutions to the BCJ equations, and whose double copy gives the desired $\Omega^{\mu} \Omega^{\nu}-\mathcal{N}^{\mu} \mathcal{N}^{\nu}$, giving further evidence that these are gravity amplitudes.

That these amplitudes containing scalars are BCJ compatible can be explained in hindsight by the fact they can be obtained from pure Yang Mills by dimensional reduction. Remarkably, the amplitude that one obtains by applying dimensional reduction to the five dimensional Yang Mills pure-gluon amplitude, calculated from the Feynman rules down to four dimensions, has the property that the numerators already satisfy the Jacobi identities. This is the only case known for a five-point amplitude and could provide insight into the structure of the space of solutions of the BCJ equations. For all the successes of color-kinematics at loop level, the current calculations are done by posing ansätze that obey all the appropriate equations. One would hope that this result could shed some light on how to construct BCJ solutions effectively at loop level.

Concerning the problem of building solutions to the BCJ equations, perhaps the most promising avenue lies in exploring the invariant space of solutions under the relabellings transforming the trivalent color factors of the amplitude into each other. Additionally, they must also have the same symmetries as the corresponding color factors. These are the solutions that have been called "virtuous" by the authors in [74]. It seems natural to assume that one should be able to find a kinematical vertex analogous to the structure constant which could be used as a building block to construct these virtuous numerators. 
The advantage of this approach is clear: finding solutions to the BCJ equations is difficult both at tree- and loop-level. Although solutions in the former case are known to exist, they quickly become intractable as the number of legs grows. At loop-level, the situation is greatly complicated by the necessity of finding consistent labellings for the loop momenta in the integrand. Furthermore, the problem of the existence of these solutions for loop amplitudes remains open.

This would provide a key understanding into the relation between gravity and Yang Mills and ultimately into each theory individually. The first obvious hope is that this might indicate that some modification of gravity that is finite and/or renormalizable. Finding proof of the existence for the BCJ equations at loop level could by itself provide enough insight into the structure, constraints and, symmetries of the theory.

Finally, the discovery of BCJ has proven instrumental in uncovering structures in string theory, in particular between open and closed strings. Ideally this understanding would also result on new calculation techniques that allow us to overcome the limitations of perturbation theory applied to string and field theories. 


\section{Appendix A}

\section{Explicit Form of The}

\section{Gravitational Amplitude}

\section{A.1 The full five-point amplitude}

To avoid cluttering the text with long expressions, in this Appendix we have collected the full form of the coefficients of the amplitude shown in Eq. 2.55). They are expressed in terms of the Sudakov decomposition for the momenta $k_{1}$, $k_{2}$ given in Eq. 2.40

$$
\begin{aligned}
& A_{k k}=\frac{i \kappa^{3}}{8}\left\{\frac{1}{\beta_{1} \alpha_{2}}-\frac{\left(1+\beta_{1}\right)}{\beta_{1}\left(\alpha_{1}+\beta_{1}\right)}-\frac{\left(1-\alpha_{2}\right)}{\alpha_{2}\left(\alpha_{2}+\beta_{2}\right)}\right\}, \\
& A_{k p}=\frac{i \kappa^{3}}{8}\left\{-\frac{\left(\alpha_{1}-\alpha_{2}\right)^{2}}{\alpha_{1} \alpha_{2} \beta_{1}}-\frac{\left(\alpha_{1}-1\right)\left(\alpha_{1}+\alpha_{2}\right)}{\alpha_{1}\left(\alpha_{1}+\beta_{1}\right)}+\frac{\left(\alpha_{2}-1\right)\left(\alpha_{1}+\alpha_{2}-2\right)}{\alpha_{2}\left(\alpha_{2}+\beta_{2}\right)}\right\}, \\
& A_{k q}=\frac{i \kappa^{3}}{8}\left\{-\frac{\left(\beta_{1}-\beta_{2}\right)^{2}}{\alpha_{2} \beta_{1} \beta_{2}}-\frac{\left(\beta_{1}+1\right)\left(\beta_{1}+\beta_{2}+2\right)}{\beta_{1}\left(\alpha_{1}+\beta_{1}\right)}+\frac{\left(\beta_{2}+1\right)\left(\beta_{1}+\beta_{2}\right)}{\beta_{2}\left(\alpha_{2}+\beta_{2}\right)}\right\}, \\
& A_{p p}=\frac{i \kappa^{3}}{8}\left\{\frac{\left(\alpha_{1}-\alpha_{2}\right)^{3}}{\alpha_{1} \alpha_{2} \beta_{1}}+\frac{4\left(\alpha_{1}-1\right)\left(\alpha_{1}-\alpha_{2}-1\right)}{\alpha_{2}\left(\beta_{2}-\beta_{1}\right)}\right.
\end{aligned}
$$




$$
\begin{aligned}
& \left.-\frac{\left(\alpha_{1}-1\right)\left(\alpha_{1}+\alpha_{2}\right)^{2}}{\alpha_{1}\left(\alpha_{1}+\beta_{1}\right)}+\frac{\left(\alpha_{2}-1\right)\left(\alpha_{1}+\alpha_{2}-2\right)^{2}}{\alpha_{2}\left(\alpha_{2}+\beta_{2}\right)}\right\}, \\
A_{q q} & =\frac{i \kappa^{3}}{8}\left\{-\frac{\left(\beta_{1}-\beta_{2}\right)^{3}}{\alpha_{2} \beta_{1} \beta_{2}}-\frac{4\left(\beta_{1}-\beta_{2}-1\right)\left(\beta_{2}+1\right)}{\left(\alpha_{1}-\alpha_{2}\right) \beta_{1}}\right. \\
& \left.-\frac{\left(\beta_{1}+1\right)\left(\beta_{1}+\beta_{2}+2\right)^{2}}{\beta_{1}\left(\alpha_{1}+\beta_{1}\right)}+\frac{\left(\beta_{2}+1\right)\left(\beta_{1}+\beta_{2}\right)^{2}}{\beta_{2}\left(\alpha_{2}+\beta_{2}\right)}\right\}, \\
A_{p q} & =\frac{i \kappa^{3}}{8}\left[\left(\alpha_{1}+\alpha_{2}-2\right)\left(\beta_{1}-\alpha_{2}\right)+\left(\alpha_{1}+\alpha_{2}\right)\left(\alpha_{2}+\beta_{2}\right)\right] \\
& \times\left\{\frac{\alpha_{1}-\alpha_{2}}{\alpha_{1} \alpha_{2} \beta_{1}}+\frac{1-\alpha_{1}}{\alpha_{1}\left(\alpha_{1}+\beta_{1}\right)}+\frac{\alpha_{2}-1}{\alpha_{2}\left(\alpha_{2}+\beta_{2}\right)}\right\} .
\end{aligned}
$$

\section{A.2 The two topologies contributing to the full amplitude}

Next we list the coefficients corresponding to the partial amplitude $\mathcal{M}_{\uparrow}$

$$
\begin{aligned}
A_{k k}^{\uparrow} & =\frac{i \kappa^{3}}{8}\left\{\frac{1}{\alpha_{2} \beta_{1}}-\frac{\left(\beta_{1}+1\right)}{\beta_{1}\left(\alpha_{2}+\beta_{1}\right)}+\frac{\alpha_{2}-1}{\alpha_{2}\left(\alpha_{2}+\beta_{2}\right)}\right\}, \\
A_{k p}^{\uparrow} & =\frac{i \kappa^{3}}{8 \alpha_{2}}\left\{\frac{\left(\alpha_{2}-1\right)\left(\alpha_{1}+\alpha_{2}-2\right)}{\alpha_{2}+\beta_{2}}-\frac{\alpha_{1}+\alpha_{2}\left(2 \alpha_{2}-3\right)}{\alpha_{2}+\beta_{1}}\right\}, \\
A_{k q}^{\uparrow} & =\frac{i \kappa^{3}}{8 \alpha_{2}}\left\{\frac{1}{\alpha_{2}+\beta_{1}}+\frac{\left(1-\alpha_{2}\right)\left(\alpha_{2}-\beta_{1}\right)}{\left(\alpha_{2}+\beta_{1}\right)\left(\alpha_{2}+\beta_{2}\right)}\right\}, \\
A_{p p}^{\uparrow} & =\frac{i \kappa^{3}}{8 \alpha_{2}}\left\{\frac{\left(\alpha_{2}-1\right)\left(\alpha_{1}+\alpha_{2}-2\right)^{2}}{\alpha_{2}+\beta_{2}}+\frac{\left(\alpha_{1}-3 \alpha_{2}\right)\left[\alpha_{2}^{2}+\left(\alpha_{1}-3\right) \alpha_{2}+\alpha_{1}\right]}{\alpha_{2}+\beta_{1}}\right. \\
& \left.-\frac{4\left(\alpha_{1}-1\right)\left(1-\alpha_{1}+\alpha_{2}\right)}{\beta_{2}-\beta_{1}}\right\}, \\
A_{q q}^{\uparrow} & =\frac{i \kappa^{3}}{8 \alpha_{2}}\left(\beta_{1}-\beta_{2}\right)\left(\beta_{1}-\beta_{2}-2 \alpha_{2}\right)\left\{\frac{\alpha_{2}+1}{\alpha_{2}+\beta_{1}}+\frac{\left(1-\alpha_{2}\right)\left(\alpha_{2}-\beta_{1}\right)}{\left(\alpha_{2}+\beta_{1}\right)\left(\alpha_{2}+\beta_{2}\right)}\right\}, \\
A_{p q}^{\uparrow} & =\frac{i \kappa^{3}}{8}\left\{\frac{\left(\alpha_{2}-1\right)\left[\beta_{1}\left(\alpha_{1}+\alpha_{2}-2\right)-2 \beta_{2}\left(\alpha_{2}-1\right)-\alpha_{2}\left(\alpha_{1}-4\right)-3 \alpha_{2}^{2}\right]}{\alpha_{2}\left(\alpha_{2}+\beta_{2}\right)}\right.
\end{aligned}
$$




$$
\left.+\frac{\alpha_{1}\left[\alpha_{2}^{2}+\left(\beta_{2}+3\right) \alpha_{2}+\beta_{2}\right]+\alpha_{2}\left[-3 \beta_{2}+\alpha_{2}\left(3 \alpha_{2}-\beta_{2}-9\right)\right]}{\alpha_{2}\left(\alpha_{2}+\beta_{1}\right)}\right\}
$$

and those of $\mathcal{M}_{\downarrow}$

$$
\begin{aligned}
& A_{k k}^{\downarrow}=\frac{i \kappa^{3}}{8}\left\{-\frac{1}{\alpha_{1} \beta_{1}}+\frac{1-\alpha_{1}}{\alpha_{1}\left(\alpha_{1}+\beta_{1}\right)}+\frac{1+\beta_{1}}{\beta_{1}\left(\alpha_{2}+\beta_{1}\right)}\right\}, \\
& A_{k p}^{\downarrow}=\frac{i \kappa^{3}}{8}\left\{-\frac{\left(\alpha_{1}-\alpha_{2}\right)^{2}}{\alpha_{1} \alpha_{2} \beta_{1}}-\frac{\left(\alpha_{1}-1\right)\left(\alpha_{1}+\alpha_{2}\right)}{\alpha_{1}\left(\alpha_{1}+\beta_{1}\right)}+\frac{\alpha_{1}+\alpha_{2}\left(2 \alpha_{2}-3\right)}{\alpha_{2}\left(\alpha_{2}+\beta_{1}\right)}\right\} \text {, } \\
& A_{k q}^{\downarrow}=\frac{i \kappa^{3}}{8}\left\{\frac{\beta_{1}\left(2 \beta_{1}+3\right)-\beta_{2}}{\beta_{1}\left(\alpha_{2}+\beta_{1}\right)}-\frac{\left(\beta_{1}+1\right)\left(\beta_{1}+\beta_{2}+2\right)}{\beta_{1}\left(\alpha_{1}+\beta_{1}\right)}\right\} \text {, } \\
& A_{p p}^{\downarrow}=\frac{i \kappa^{3}}{8}\left\{\frac{\left(\alpha_{1}-\alpha_{2}\right)^{3}}{\alpha_{1} \alpha_{2} \beta_{1}}-\frac{\left(\alpha_{1}-1\right)\left(\alpha_{1}+\alpha_{2}\right)^{2}}{\alpha_{1}\left(\alpha_{1}+\beta_{1}\right)}\right. \\
& \left.-\frac{\left(\alpha_{1}-3 \alpha_{2}\right)\left[\alpha_{2}^{2}+\left(\alpha_{1}-3\right) \alpha_{2}+\alpha_{1}\right]}{\alpha_{2}\left(\alpha_{2}+\beta_{1}\right)}\right\} \text {, } \\
& A_{q q}^{\downarrow}=\frac{i \kappa^{3}}{8}\left\{-\frac{\left(\beta_{1}+1\right)\left(\beta_{1}+\beta_{2}+2\right)^{2}}{\beta_{1}\left(\alpha_{1}+\beta_{1}\right)}-\frac{4\left(\beta_{1}-\beta_{2}-1\right)\left(\beta_{2}+1\right)}{\left(\alpha_{1}-\alpha_{2}\right) \beta_{1}}\right. \\
& \left.+\frac{\left(3 \beta_{1}-\beta_{2}\right)\left[\beta_{1}\left(\beta_{1}+\beta_{2}+3\right)-\beta_{2}\right]}{\beta_{1}\left(\alpha_{2}+\beta_{1}\right)}\right\} \text {, } \\
& A_{p q}^{\downarrow}=\frac{i \kappa^{3}}{8}\left\{\frac{\left(\beta_{1}+1\right)\left[\beta_{1}\left(-\alpha_{2}+\beta_{2}+4\right)+2 \alpha_{1}\left(\beta_{1}+1\right)-\alpha_{2}\left(\beta_{2}+2\right)+3 \beta_{1}^{2}\right]}{\beta_{1}\left(\alpha_{1}+\beta_{1}\right)}\right. \\
& \left.+\frac{\alpha_{1}\left[\beta_{1}^{2}-\left(\beta_{2}+3\right) \beta_{1}+\beta_{2}\right]+\beta_{1}\left[3 \beta_{2}-\beta_{1}\left(3 \beta_{1}+\beta_{2}+9\right)\right]}{\beta_{1}\left(\alpha_{2}+\beta_{1}\right)}\right\} .
\end{aligned}
$$




\section{Bibliography}

[1] J. M. Maldacena, The Large $N$ limit of superconformal field theories and supergravity, Adv. Theor. Math. Phys. 2 (1998) 231. [arXiv:hep-th/9711200]. [page 1]

[2] S. S. Gubser, I. R. Klebanov and A. M. Polyakov, Gauge theory correlators from noncritical string theory, Phys. Lett. B428 (1998) 105. [arXiv:hep-th/9802109]. [page 1]

[3] E. Witten, Anti-de Sitter space and holography, Adv. Theor. Math. Phys. 2 (1998) 253. [arXiv:hep-th/9802150]. [page1]

[4] Z. Bern, J. J. M. Carrasco, L. J. Dixon, H. Johansson and R. Roiban, The Complete Four-Loop Four-Point Amplitude in $\mathcal{N}=4$ Super-Yang-Mills Theory, Phys. Rev. D82 (2010) 125040. [arXiv:1008.3327 [hep-th]]. [page 1

[5] Z. Bern, L. J. Dixon, D. C. Dunbar, M. Perelstein and J. S. Rozowsky, On the relationship between Yang-Mills theory and gravity and its implication for ultraviolet divergences, Nucl. Phys. B530 (1998) 401. [arXiv:hep-th/9802162]. [page 1] 
[6] Z. Bern, J. J. Carrasco, L. J. Dixon, H. Johansson and R. Roiban, The Ultraviolet Behavior of $\mathcal{N}=8$ Supergravity at Four Loops, Phys. Rev. Lett. 103 (2009) 081301. [arXiv:0905.2326 [hep-th]]. [page 1]

[7] J. F. Donoghue, General Relativity As An Effective Field Theory: The Leading Quantum Corrections, Phys. Rev. D50 (1994) 3874. [arXiv:gr-qc/9405057]. [page 1]

[8] N. E. J. Bjerrum-Bohr, J. F. Donoghue and B. R. Holstein, Quantum gravitational corrections to the nonrelativistic scattering potential of two masses, Phys. Rev. D67 (2003) 084033 [Erratum-ibid. D71 (2005) 069903]. [arXiv:hep-th/0211072]. [page 1]

[9] J. F. Donoghue and T. Torma, Infrared behavior of graviton-graviton scattering, Phys. Rev. D60 (1999) 024003. [arXiv:hep-th/9901156]. [page 1

[10] D. C. Dunbar and P. S. Norridge, Calculation of graviton scattering amplitudes using string based methods, Nucl. Phys. B433 (1995) 181. [arXiv:hep-th/9408014]. [page 1], [page 9]

[11] Z. Bern, D. C. Dunbar and T. Shimada, String based methods in perturbative gravity, Phys. Lett. B312 (1993) 277. [arXiv:hep-th/9307001]. [page 1], [page 9]

[12] Z. Bern and D. C. Dunbar, A Mapping between Feynman and string motivated one loop rules in gauge theories, Nucl. Phys. B379 (1992) 562. [page 1], [page 9]

[13] S.-Q. Su, Graviton Bremsstrahlung at High Energies, Doctoral Thesis, Katholieke Universiteit Leuven 1982. [page 1] 
[14] J. Geris and S.-Q. Su, Single bremsstrahlung processes in quantum gravity, Commun. Theor. Phys. 8 (1987) 325. [page 1]

[15] J. F. Donoghue, Introduction to the Effective Field Theory Description of Gravity, arXiv:gr-qc/9512024. [page 1]

[16] L. F. Abbott, Introduction to the Background Field Method, Acta Phys. Polon. B13 (1982) 33. [page 2]

[17] C. W. Misner, K. S. Thorne and J. A. Wheeler, Gravitation, San Francisco 1973 [page 2]

[18] B. Delamotte, A Hint of renormalization, Am. J. Phys. 72 (2004) 170 [hepth/0212049]. [page 4]

[19] Z. Bern, L. J. Dixon and R. Roiban, Is $N=8$ supergravity ultraviolet finite?, Phys. Lett. B644 (2007) 265 [hep-th/0611086]. [page4]

[20] L. N. Lipatov, Reggeization of the Vector Meson and the Vacuum Singularity in Nonabelian Gauge Theories Sov. J. Nucl. Phys. 23 (1976) 338. [page 6], [page 9], [page 45], [page 59]

[21] E. A. Kuraev, L. N. Lipatov and V. S. Fadin, On the Pomeranchuk Singularity in Asymptotically Free Theories, Phys. Lett. B60 (1975) 50.

E. A. Kuraev, L. N. Lipatov and V. S. Fadin, Multi-Reggeon Processes in the Yang-Mills Theory, Sov. Phys. JETP 44 (1976) 443.

E. A. Kuraev, L. N. Lipatov and V. S. Fadin, The Pomeranchuk Singularity in Nonabelian Gauge Theories, Sov. Phys. JETP 45 (1977) 199. [page 6], [page 9], [page 45], [page 59] 
[22] I. I. Balitsky and L. N. Lipatov, The Pomeranchuk Singularity in Quantum Chromodynamics, Sov. J. Nucl. Phys. 28 (1978) 822. [page 6], [page 9], [page 45], [page 59

[23] L. N. Lipatov, Graviton Reggeization, Phys. Lett. B116 (1982) 411. [page 8], [page 9], [page 20], [page 37, [page 41], [page 45], [page 59]

[24] A. Sabio Vera, E. Serna Campillo and M. A. Vázquez-Mozo, Graviton emission in Einstein-Hilbert gravity, J. High Energy Phys. 03 (2012) 005 [arXiv: 1112.4494 [hep-th]] . [page 8], [page 45], [page 52], [page 59]

[25] L. N. Lipatov, Effective action for the Regge processes in gravity, arXiv:1105.3127 [hep-th]. [page 8], [page 9], [page 41], [page 45], [page 59

[26] M. T. Grisaru, P. van Nieuwenhuizen and C. C. Wu, Reggeization and the Question of Higher Loop Renormalizability of Gravitation, Phys. Rev. D12 (1975) 1563. [page 8]

[27] M. T. Grisaru and H. J. Schnitzer, Dynamical Calculation Of Bound State Supermultiplets In $\mathcal{N}=8$ Supergravity, Phys. Lett. B107 (1981) 196. [page 8

[28] L. N. Lipatov, Multi-Regge Processes In Gravitation, Sov. Phys. JETP 55 (1982) 582 [Zh. Eksp. Teor. Fiz. 82 (1982) 991]. [page 8], [page 9], [page 20], [page 37, [page 41, [page 45, [page 59]

[29] L. N. Lipatov, High-energy scattering in QCD and in quantum gravity and two-dimensional field theories, Nucl. Phys. B365 (1991) 614. [page 8], [page 20], [page 37, [page 41], [page 45], [page 59] 
[30] D. J. Gross and P. F. Mende, The High-Energy Behavior of String Scattering Amplitudes, Phys. Lett. B197 (1987) 129. D. J. Gross and P. F. Mende, String Theory Beyond the Planck Scale, Nucl. Phys. B303 (1988) 407. [page 9

[31] D. J. Gross and J. L. Mañes, The High-energy Behavior of Open String Scattering, Nucl. Phys. B326 (1989) 73. [page 9

[32] Z. Bern, Perturbative quantum gravity and its relation to gauge theory, Living Rev. Rel. 5 (2002) 5 [gr-qc/0206071] .

J. J. M. Carrasco and H. Johansson, Generic multiloop methods and application to $\mathcal{N}=4$ super-Yang-Mills, J. Phys. A44 (2011) 454004 [arXiv:1103.3298 [hep-th]]. [page9]

[33] H. Kawai, D. C. Lewellen and S. H. H. Tye, A Relation Between Tree Amplitudes of Closed and Open Strings, Nucl. Phys. B269 (1986) 1. [page 9

[34] H. Elvang and Y. t. Huang, Scattering Amplitudes, arXiv:1308.1697 [hep-th]. [page 9]

[35] Z. Bern, J. J. M. Carrasco and H. Johansson, New Relations for GaugeTheory Amplitudes, Phys. Rev. D78 (2008) 085011 [arXiv:0805.3993 [hep-ph]]. [page 10, [page 43], [page 44], [page 45], [page 71]

[36] Z. Bern, J. J. M. Carrasco and H. Johansson, Perturbative Quantum Gravity as a Double Copy of Gauge Theory, Phys. Rev. Lett. 105 (2010) 061602. [arXiv:1004.0476 [hep-th]]. [page 10, [page 37, [page 43]

[37] Z. Bern, T. Dennen, A Color Dual Form for Gauge-Theory Amplitudes, arXiv:1103.0312 [hep-th]. [page 10, [page 37, [page 43 
[38] Z. Bern, T. Dennen, Y.-t. Huang and M. Kiermaier, Gravity as the Square of Gauge Theory, Phys. Rev. D82 (2010) 065003 [arXiv:1004.0693 [hep-th] ] . [page 10], [page 43], [page 44], [page 59], [page 71]

[39] S. H. Henry Tye and Y. Zhang, Dual Identities inside the Gluon and the Graviton Scattering Amplitudes, J. High Energy Phys. 06 (2010) 071 [Erratum-ibid. 04 (2011) 114] [arXiv:1003.1732 [hep-th]]. [page 10, [page 43

[40] C. R. Mafra, O. Schlotterer and S. Stieberger, Explicit BCJ Numerators from Pure Spinors, J. High Energy Phys. 07 (2011) 092 [arXiv:1104.5224 [hep-th]]. [page 10], [page 43

[41] R. Kleiss and H. Kuijf, Multi - Gluon Cross-sections and Five Jet Production at Hadron Colliders, Nucl. Phys. B312 (1989) 616. [page 14

[42] N. E. J. Bjerrum-Bohr, P. H. Damgaard and P. Vanhove, Minimal Basis for Gauge Theory Amplitudes, Phys. Rev. Lett. 103 (2009) 161602 [arXiv:0907.1425 [hep-th]]. [page 14]

[43] B. Feng, R. Huang and Y. Jia, Gauge Amplitude Identities by On-shell Recursion Relation in S-matrix Program, Phys. Lett. B695 (2011) 350 [arXiv:1004.3417 [hep-th]]. [page 14

[44] N. E. J. Bjerrum-Bohr, P. H. Damgaard, T. Sondergaard and P. Vanhove, The Momentum Kernel of Gauge and Gravity Theories, J. High Energy Phys. 1101 (2011) 001 [arXiv:1010.3933 [hep-th]] . [page 14]

[45] R. Britto, F. Cachazo, B. Feng and E. Witten, Direct proof of tree-level recursion relation in Yang-Mills theory, Phys. Rev. Lett. 94 (2005) 181602 [hep-th/0501052]. [page 15] 
[46] Z. Bern, C. Boucher-Veronneau and H. Johansson, $N>=4$ Supergravity Amplitudes from Gauge Theory at One Loop, Phys. Rev. D84 (2011) 105035 [arXiv:1107.1935 [hep-th]]. [page 16

[47] Z. Bern and Y. t. Huang, Basics of Generalized Unitarity, J. Phys. A44 (2011) 454003 [arXiv:1103.1869 [hep-th]] . [page 17

[48] Z. Bern, L. J. Dixon, D. C. Dunbar and D. A. Kosower, One loop n point gauge theory amplitudes, unitarity and collinear limits, Nucl. Phys. B425 (1994) 217 [hep-ph/9403226]. [page 17]

[49] Z. Bern, L. J. Dixon, D. C. Dunbar and D. A. Kosower, Fusing gauge theory tree amplitudes into loop amplitudes, Nucl. Phys. B435 (1995) 59 [hepph/9409265]. [page 17

[50] Z. Bern, L. J. Dixon and D. A. Kosower, Two-loop $g \rightarrow$ gg splitting amplitudes in QCD, J. High Energy Phys. 408 (2004) 012 [hep-ph/0404293]. [page 17]

[51] J. M. Martín-García, xPerm: fast index canonicalization for tensor computer algebra, Comput. Phys. Commun. 179 (2008) 597. [page 19], [page 32]

[52] Z. Bern, Perturbative quantum gravity and its relation to gauge theory, Living Rev. Rel. 5 (2002) 5. [arXiv:gr-qc/0206071].

J. J. M. Carrasco and H. Johansson, Generic multiloop methods and application to $\mathcal{N}=4$ super-Yang-Mills, J. Phys. A44 (2011) 454004 [arXiv:1103.3298 [hep-th]]. [page 20, [page 37]

[53] L. D. Landau and E. M. Lifshitz, The Classical Theory of Fields, 3rd revised edition, Pergamon 1971. [page 22 
[54] B. S. DeWitt, Quantum theory of gravity. III. Applications of the covariant theory, Phys. Rev. 162 (1967) 1239. [page 23]

[55] R. Gastmans and T. T. Wu, The Ubiquitous Photon: Helicity Method for QED and QCD, Oxford 1990. [page 25

[56] Z. Xu, D. H. Zhang and L. Chang, Helicity Amplitudes for Multiple Bremsstrahlung in Massless Nonabelian Gauge Theories, Nucl. Phys. B291 (1987) 392. [page 26

[57] O. Steinmann, Über den Zusammenhang zwischen den Wightmanfunktionen und der retardierten Kommutatoren, Helv. Phys. Acta 33 (1960) 257, O. Steinmann, Wightman-Funktionen und retardierten Kommutatoren. II, Helv. Phys. Acta. 33 (1960) 347. [page 34], [page 53], [page 59]

[58] Z. Bern, T. Dennen, Y.-t. Huang and M. Kiermaier, Gravity as the Square of Gauge Theory, Phys. Rev. D82 (2010) 065003. [arXiv:1004.0693 [hep-th] ]. [page 37]

[59] S. Oxburgh and C. D. White, BCJ duality and the double copy in the soft limit, arXiv:1210.1110 [hep-th] . [page 45

[60] J. Bartels, L. N. Lipatov and A. Sabio Vera, Double-logarithms in EinsteinHilbert gravity and supergravity, J. High Energy Phys. 07 (2014) 056 arXiv:1208.3423 [hep-th] . [page 45, [page 59]

[61] J. J. Carrasco and H. Johansson, Five-Point Amplitudes in N=4 SuperYang-Mills Theory and $\mathcal{N}=8$ Supergravity, Phys. Rev. D85 (2012) 025006 [arXiv:1106.4711 [hep-th]].

Z. Bern, C. Boucher-Veronneau and H. Johansson, $\mathcal{N} \geq 4$ Supergravity Amp- 
litudes from Gauge Theory at One Loop, Phys. Rev. D84 (2011) 105035 [arXiv:1107.1935 [hep-th]]. [page 45]

[62] A. Sabio Vera, E. Serna Campillo and M. A. Vázquez-Mozo, ColorKinematics Duality and the Regge Limit of Inelastic Amplitudes, J. High Energy Phys. 04 (2013) 086 [arXiv:1212.5103 [hep-th]]. [page 46]

[63] C. Itzykson and J. B. Zuber, Quantum Field Theory, McGraw-Hill 1980. [page 48]

[64] J. Bartels, L. N. Lipatov and A. Sabio Vera, BFKL Pomeron, Reggeized gluons and Bern-Dixon-Smirnov amplitudes, Phys. Rev. D80 (2009) 045002. [arXiv:0802.2065 [hep-th]]. [page 53, [page 59

[65] J. Bartels, L. N. Lipatov and A. Sabio Vera, $\mathcal{N}=4$ supersymmetric Yang Mills scattering amplitudes at high energies: The Regge cut contribution, Eur. Phys. J. C65 (2010) 587. [arXiv:0807.0894 [hep-th]]. [page 53, [page 59]

[66] H. Johansson, A. Sabio Vera, E. Serna Campillo and M. Á. VázquezMozo, Color-Kinematics Duality in Multi-Regge Kinematics and Dimensional Reduction, J. High Energy Phys. 10 (2013) 215 [arXiv:1307.3106 [hep-th]] . [page 60]

[67] Z. Bern, J. J. M. Carrasco and H. Johansson, Perturbative Quantum Gravity as a Double Copy of Gauge Theory, Phys. Rev. Lett. 105 (2010) 061602. [arXiv:1004.0476 [hep-th]]. [page 71]

[68] J. J. Carrasco and H. Johansson, Five-Point Amplitudes in N=4 SuperYang-Mills Theory and N=8 Supergravity, Phys. Rev. D85 (2012) 025006 [arXiv:1106.4711 [hep-th]]; 
Z. Bern, J. J. M. Carrasco, L. J. Dixon, H. Johansson and R. Roiban, Simplifying Multiloop Integrands and Ultraviolet Divergences of Gauge Theory and Gravity Amplitudes, Phys. Rev. D85 (2012) 105014 [arXiv:1201.5366 [hep-th]] . [page 71]

[69] R. H. Boels, B. A. Kniehl, O. V. Tarasov and G. Yang, Color-kinematic Duality for Form Factors, J. High Energy Phys. 02 (2013) 063 [arXiv: 1211.7028 [hep-th]];

J. J. M. Carrasco, M. Chiodaroli, M. Gunaydin and R. Roiban, One-loop four-point amplitudes in pure and matter-coupled $N \leq 4$ supergravity, J. High Energy Phys. 03 (2013) 056 [arXiv:1212.1146 [hep-th]];

R. H. Boels, R. S. Isermann, R. and D. O'Connell, Colour-Kinematics Duality for One-Loop Rational Amplitudes, J. High Energy Phys 04 (2013) 107 [arXiv:1301.4165 [hep-th]];

N. E. J. Bjerrum-Bohr, T. Dennen, R. Monteiro and D. O'Connell, Integrand Oxidation and One-Loop Colour-Dual Numerators in N=4 Gauge Theory, J. High Energy Phys. 07 (2013) 092 [arXiv:1303.2913 [hep-th]]. Z. Bern, S. Davies, T. Dennen, Y. -t. Huang and J. Nohle, Color-Kinematics Duality for Pure Yang-Mills and Gravity at One and Two Loops, arXiv:1303.6605 [hep-th] . [page 71]

[70] Z. Bern, C. Boucher-Veronneau and H. Johansson, $N \geq 4$ Supergravity Amplitudes from Gauge Theory at One Loop, Phys. Rev. D84 (2011) 105035 [arXiv:1107.1935 [hep-th]];

C. Boucher-Veronneau and L. J. Dixon, $N \geq 4$ Supergravity Amplitudes from Gauge Theory at Two Loops, J. High Energy Phys. 12 (2011) 046 [arXiv:1110.1132 [hep-th]]. [page 71] 
[71] Z. Bern, S. Davies, T. Dennen and Y. -t. Huang, Absence of Three-Loop Four-Point Divergences in $N=4$ Supergravity, Phys. Rev. Lett. 108 (2012) 201301 [arXiv:1202.3423 [hep-th]];

Z. Bern, S. Davies, T. Dennen and Y. -t. Huang, Ultraviolet Cancellations in Half-Maximal Supergravity as a Consequence of the Double-Copy Structure, Phys. Rev. D86 (2012) 105014 [arXiv:1209.2472 [hep-th]];

Z. Bern, S. Davies and T. Dennen, The Ultraviolet Structure of HalfMaximal Supergravity with Matter Multiplets at Two and Three Loops, arXiv: 1305.4876 [hep-th]. [page 71]

[72] M. A. Vázquez-Mozo, A Note on supersymmetric Yang-Mills thermodynamics, Phys. Rev. D60 (1999) 106010 [hep-th/9905030] . [page 71]

[73] M. Chiodaroli, M. Gunaydin, H. Johansson and R. Roiban, Scattering amplitudes in N=2 Maxwell-Einstein and Yang-Mills/Einstein supergravity, arXiv: 1408.0764 [hep-th]. [page 71]

[74] J. Broedel and J. J. M. Carrasco, Virtuous Trees at Five and Six Points for Yang-Mills and Gravity, Phys. Rev. D84 (2011) 085009 [arXiv:1107.4802 [hep-th]] . [page 74 